\title{
WestVirginiaUniversity
}

THE RESEARCH REPOSITORY @ WVU

Graduate Theses, Dissertations, and Problem Reports

2007

\section{De-wetting of cobalt thin films on sapphire}

Jorge Espinosa

West Virginia University

Follow this and additional works at: https://researchrepository.wvu.edu/etd

\section{Recommended Citation}

Espinosa, Jorge, "De-wetting of cobalt thin films on sapphire" (2007). Graduate Theses, Dissertations, and Problem Reports. 2579.

https://researchrepository.wvu.edu/etd/2579

This Dissertation is protected by copyright and/or related rights. It has been brought to you by the The Research Repository @ WVU with permission from the rights-holder(s). You are free to use this Dissertation in any way that is permitted by the copyright and related rights legislation that applies to your use. For other uses you must obtain permission from the rights-holder(s) directly, unless additional rights are indicated by a Creative Commons license in the record and/ or on the work itself. This Dissertation has been accepted for inclusion in WVU Graduate Theses, Dissertations, and Problem Reports collection by an authorized administrator of The Research Repository @ WVU.

For more information, please contact researchrepository@mail.wvu.edu. 


\title{
DE-WETTING OF COBALT THIN FILMS ON SAPPHIRE
}

\author{
By Jorge Espinosa \\ DISSERTATION \\ Submitted to the Eberly College of Art and Sciences \\ at \\ West Virginia University \\ in partial fulfillment of the requirements for the degree of \\ Doctor of Philosophy \\ In Physics \\ David Lederman, Ph D., Chair \\ Leonardo Golubovic, Ph. D. \\ Mohindar Seehra, Ph. D. \\ Sergei Urazhdin, Ph. D. \\ Charter Stinespring, Ph. D. \\ Department of Physics \\ Morgantown, West Virginia University
}

2007

Keywords:

De-wetting, cobalt, sapphire, roughening, cluster, annealing, AFM, surface energy, Casimir interaction, diffusion 


\section{ABSTRACT \\ DE-WETTING OF COBALT THIN FILMS ON SAPPHIRE}

\section{Jorge Espinosa}

Cobalt thin films $4 \mathrm{~nm}$ thick were deposited via metal electron-beam epitaxy on sapphire (110). The films were annealed above $500{ }^{\circ} \mathrm{C}$ and scanned in-situ with atomic force microscopy upon annealing. The films underwent a roughening transition above $500{ }^{\circ} \mathrm{C}$ which is highly time dependent. The film's roughness increased with time with a power law of $\rho \sim\left(t-t_{0}\right)^{\alpha}$, with $\alpha$ ranging from 0.16 to 0.21 , and de-wetted the sapphire substrate above $590{ }^{\circ} \mathrm{C}$. The dynamics of single clusters showed an exponential growth of their height with increasing time at early stages of the annealing process which is consistent with surface instabilities. These instabilities are due to the interplay between surface tension forces (which favor the smoothing out of the surface) and long range Casimir interactions (which favor the thickening and thus roughening of the film). The nature of the Casimir interaction acting within the metallic film was analyzed by calculating the average intercluster separation at early annealing times. The images' autocorrelation function gave an intercluster separation between 150$300 \mathrm{~nm}$, which agrees with expectations resulting from a fermionic Casimir interaction. The fermionic Casimir interaction should be more important for the de-wetting of metallic thin films like cobalt rather than the bosonic Casimir interaction (Van der Waals) that plays an important role in the de-wetting of insulators like polymers. The clusters' evolution for late annealing times showed mainly a growth perpendicular to the surface plane with their areas remaining almost unchanged. The clusters' height increased with a power law $h \sim\left(t-t_{0}\right)^{\beta}$ with $\beta \sim 0.25$, which in principle is consistent with diffusion in 2 dimensions for a 3 dimensional system (3D/2D), however coalescence and ripening are not observed in the evolution of cobalt thin films. Hence the standard Ostwald ripening theory does not seem to fit well in this system. 


\section{DEDICATION}

To my parents Carmen and Jorge and to my grandparents Benita, María, Danilo and Enrique. 


\section{ACKNOWLEDGMENTS}

I thank my advisor Dr. David Lederman for his support during these years, for helping me to solve many experimental problems and pushing me to finish this project. I thank Dr. Golubovic for helping me to understand the theory behind this work and all the faculties in the Department specially Dr. Seehra, Dr. Halliburton and Dr. Myers. I thank Dr. Stinespring for allowing me to anneal some substrates in his laboratory and his postdoc Andy that made that possible. The students in this department are wonderful and they have been a little family for me, specially Vivek, Eduardo, our postdoc Dr. Miyeon Cheon, Guerito, Matias, Dr. Gu, Debin and all the people that is not at WVU anymore such as: Hongtao, Erie, Dr. Liu, etc. I am very thankful of the Physics Department Staff and the people of the machine shop. I thank my family that support me through the distance, Carmen and Nano, Lala, Kike, Moises, Sandra, Macarena, Alejandro, Sebastian, Sylvia, Kihana and Itzel. Finally I thank NSF for founding this project. 


\section{INDEX OF CONTENTS}

1 INTRODUCTION..........................................................1

2 DE-WETTING OVERVIEW ............................................2

3 MOTIVATION FOR STUDING COBALT THIN FILMS ....................6

4 BASIC CONCEPTS....................................................8

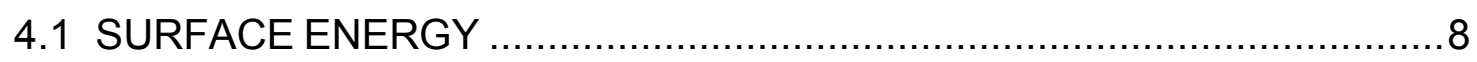

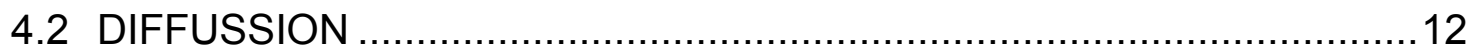

$5 \quad$ CLUSTER RIPENING ............................................... 14

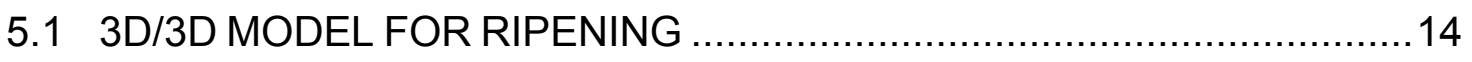

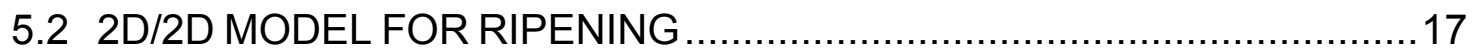

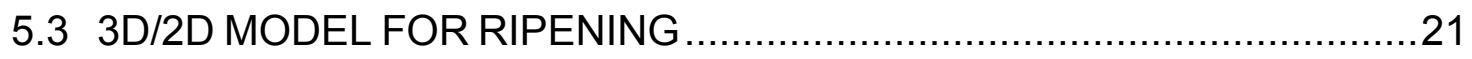

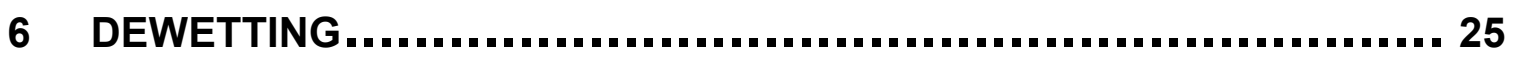

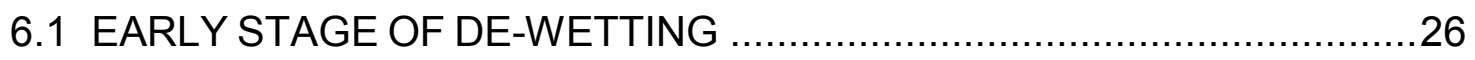

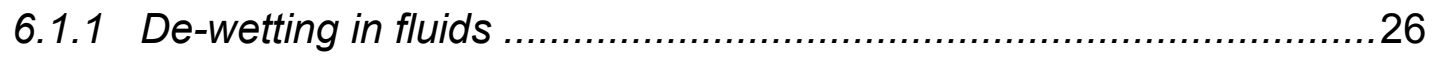

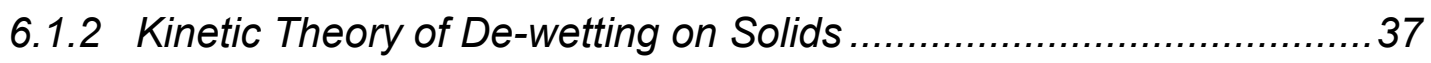

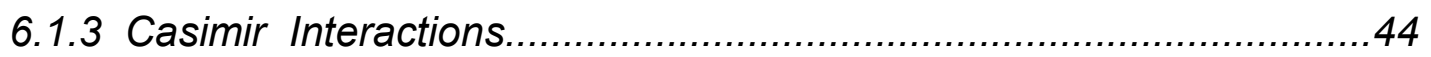

6.2 INTERMEDIATE AND LATE STAGES OF DE-WETTING .....................46

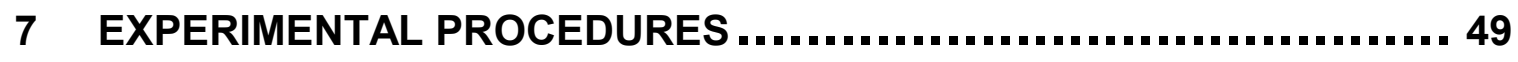

7.1 SUBSTRATE PREPARATION AND CLEANING .................................50

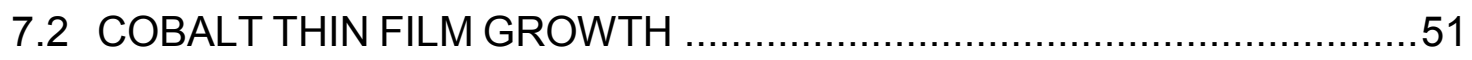

7.3 IMAGING OF THE FILM UPON ANNEALING …................................53

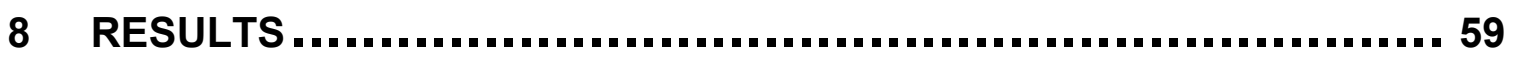

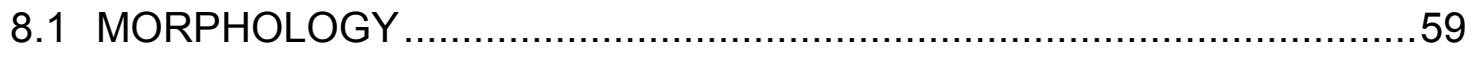

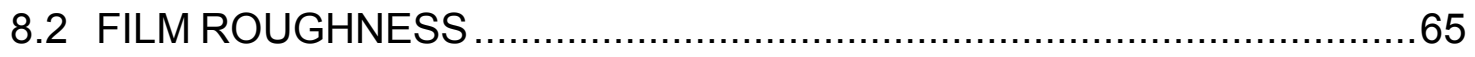

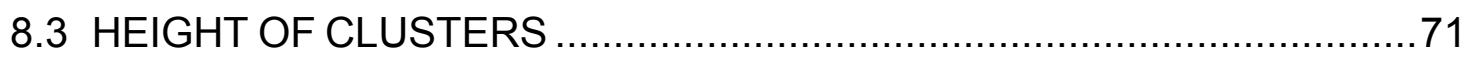

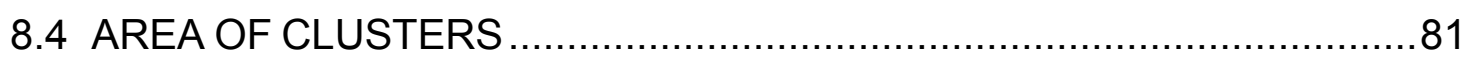

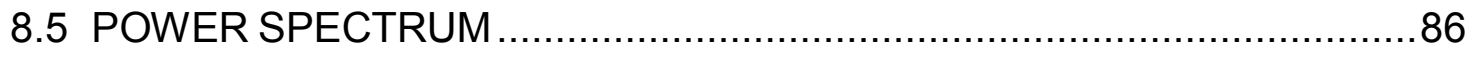


9 DISCUSION ............................................................. 97

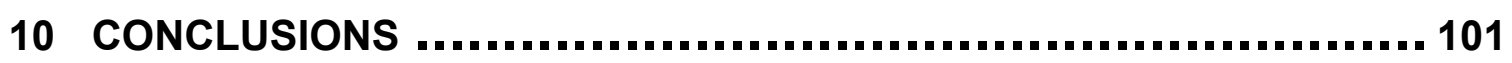

REFERENCES ............................................................ 102

\section{INDEX OF FIGURES}

FIGURE 1 PROCESSES THIN FILMS TOWARDS STABLE STATES. ............................ 4

FIGURE 2. CLUSTERS UNDERGOING DIFFERENT RIPENING PROCESSES: ...................5

FIGURE 3. EX-SITU AFM IMAGE OF A COBALT THIN FILM (5 NM THICK) ON SAPPHIRE

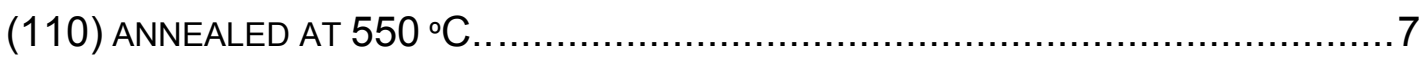

FIGURE 4. AFM IMAGES OF A 5 NM COBALT THIN FILM ON SAPPHIRE (110) ANNEALED AT

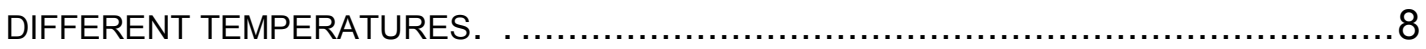

FIGURE 5. FORMATION OF A UNIT OF AREA A FOR A MONOATOMIC SOLID...................9

FIGURE 6. SURFACE ENERGY FOR SEVERAL 3D TRANSITION METALS. DATA TAKEN FROM

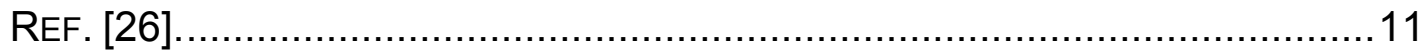

FIGURE 7. WULFF CONSTRUCTION FOR BCC FE IN THE (001) PLANE.......................12

FIGURE 8. STM IMAGE SHOWING THE DECAY OF A 2D Ag CLUSTER ON Ag (111) AT

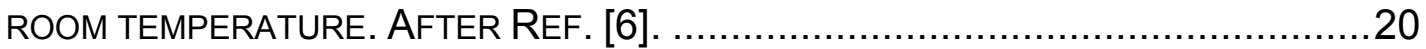

FIGURE 9. DECAY OF THE RADIUS FOR A 2D Ag ISLAND ON Ag (111). ..................20

FIGURE 10. STM IMAGES OF MG SILICIDE ISLANDS ON SI (001) ANNEALED AT $500^{\circ} \mathrm{C}$

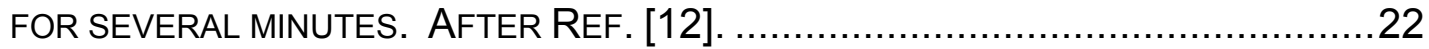

Figure 11. AVERAgE CLUSTER'S HEIGHT H AS A FUNCTION OF TIME FOR Mg SILICIDE ISLANDS ON SI (001).

FIGURE 12. SKETCH OF A DROP ON TOP OF A SURFACE DEPICTING THE YOUNG-DUPRE

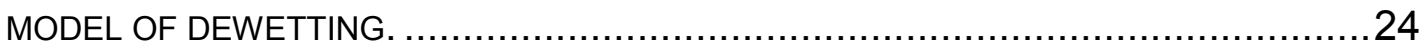

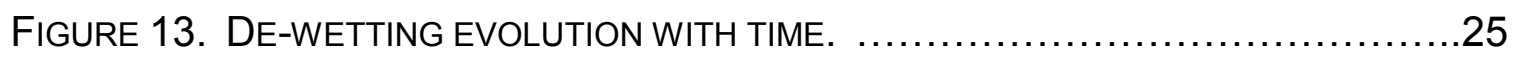

FIGURE 14. ONE FOURIER COMPONENTS WITH WAVELENGTH $\wedge$ AND AMPLITUDE $B$ OF A CORRUGATED THIN FILM DUE TO SURFACE INSTABILITIES. 
FiguRE 15. DifFERENCES BETWEEN EFFECTIVE INTERFACIAL POTENTIAL IN A FILM. . . 30

FIGURE 16. AFM IMAGES OF A PS FILM ANNEALED AT $115^{\circ} \mathrm{C}$ 32

FIGURE 17. RADIAL AVERAGE OF THE POWER SPECTRUM AS A FUNCTION OF THE WAVE VECTOR FOR A PS FILM $45 \AA$ THICK.. 34

FIGURE 18. (A) CHARACTERISTIC WAVELENGTH OF A PS FILM AS A FUNCTION OF THICKNESS. 35

FIgURE 19. AVERAGE AMPLITUdE OF THE CORRUGATIONS FOR A PS FILM (45 Á THICK) anNeAled at $115^{\circ} \mathrm{C}$. After Ref. [5]. 37

FIGURE 20. VARIATION OF THE FREQUENCY WITH THE CHARACTERISTIC WAVE LENGTH OF THE CORRUGATIONS IN A THIN FILM. 41

FIGURE 21. PS FILM (3.9 NM THICK) ON TOP OF SIO ANNEALED AT $53^{\circ} \mathrm{C} . \ldots \ldots \ldots . . . . .46$

FIGURE 22. AFM IMAGE OF A PS FILM 41 NM THICK.....................................4

FIGURE 23. LATE STAGE OF DE-WETTING OF A PS FILM ON SILICON.. …..................48

FIGURE 24. SEM IMAGE SHOWING THE DE-WETTING OF A 500 NM ALUMINUM THIN FILM ON SAPPHIRE (001) ANNEALED AT $900^{\circ} \mathrm{C}$ DURING 120 MINUTES......................48

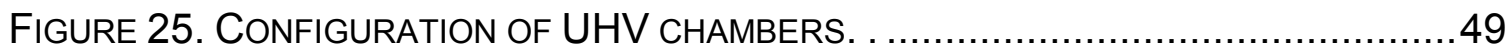

FIGURE 26. ACTUAL PICTURE OF THE MBE CHAMBER. .....................................50

FIGURE 27. CALIBRATION OF TEMPERATURE FOR THE OMICRON INDIRECT HEATER.. ..55

FIGURE 28. SCANNING ELECTRON MICROSCOPY IMAGES OF A NEEDLE SENSOR TIP. ...57

FIGURE 29. VARIATION OF THE RESONANCE FREQUENCY FOR THE NEEDLE TIP AT

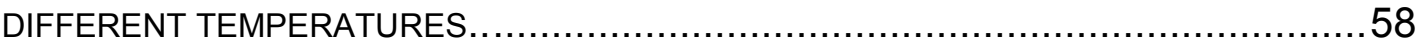

FIGURE 30. (A) SAPPHIRE (11-20) BEFORE GROWTH. (B) 4 NM THICK COBALT FILM

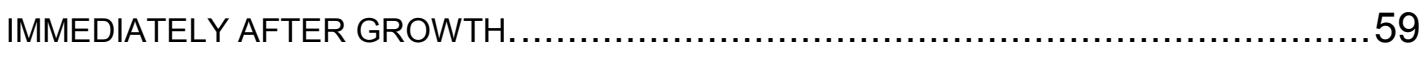

FIGURE 31. AFM IMAGES OF A COBALT FILM (4 NM THICK) ON $\mathrm{AL}_{2} \mathrm{O}_{3}$ (110) ANNEALED FOR 16 HOURS AT $535^{\circ} \mathrm{C}$.

FIGURE 32. AFM IMAGES OF A COBALT FILM ANNEALED AT $565^{\circ} \mathrm{C}$ FOR SEVERAL HOURS..

FIGURE 33. AFM IMAGE OF COBALT FILM ON SAPPHIRE (110) ANNEALED AT A CONSTANT TEMPERATURE OF $590^{\circ} \mathrm{C}$..

FIGURE 34. 3D IMAGES FOR COBALT FILM ANNEALED AT $535^{\circ} \mathrm{C}$ AT DIFFERENT ANNEALING TIMES. 
FIGURE 35. 3D IMAGES OF COBALT FILM ANNEALED AT $565^{\circ} \mathrm{C}$ AT DIFFERENT ANNEALING TIMES.

FIGURE 36. ROUGHNESS AS A FUNCTION OF TIME (RED DOTS) FOR THE ANNEALED FILMS

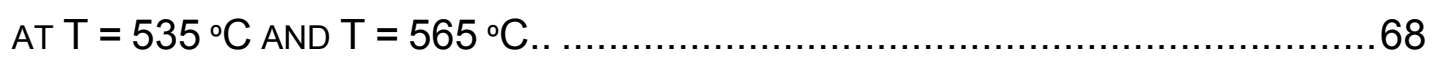

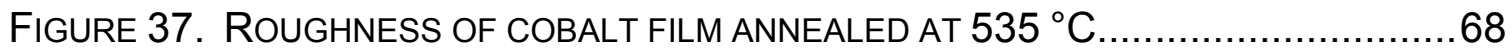

FIGURE 38. ROUGHNESS AS A FUNCTION OF TIME (BLUE DOTS) FOR THE FILM ANNEALED

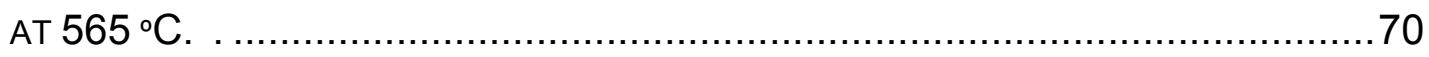

FIGURE 39. ROUGHNESS AS A FUNCTION OF TIME (BLUE DOTS) FOR THE FILM ANNEALED

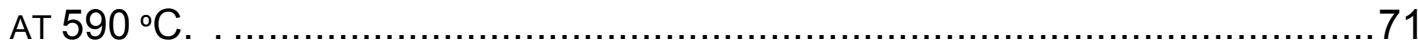

FIGURE 40. AFM IMAGE OF COBALT FILM ANNEALED AT $535^{\circ} \mathrm{C}$ FOR $7.5 \mathrm{HR} . \ldots \ldots \ldots . . .73$ FIGURE 41. HEIGHT AS A FUNCTION OF TIME FOR 3 DIFFERENT CLUSTERS ANNEALED AT

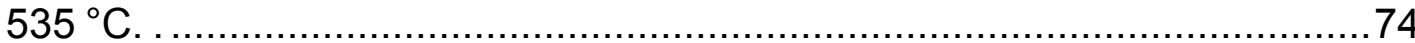

FIGURE 42. GROWTH OF THE CLUSTER HEIGHT WITH TIME FOR 2 DIFFERENT ANNEALING

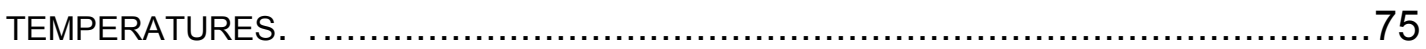

FIGURE 43. (A) CLUSTER HEIGHT AS A FUNCTION OF ANNEALING TIME FOR A COBALT

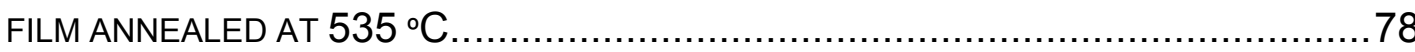

FiguRE 44. CLUSTER HEIGHT AS A FUNCTION OF ANNEALING TIME FOR A TEMPERATURE

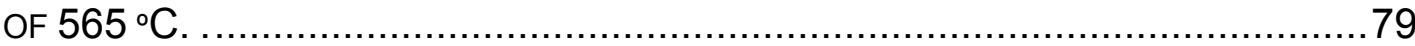

FIGURE 45. (A) CLUSTER HEIGHT AS A FUNCTION OF TIME FOR A COBALT FILM ANNEALED

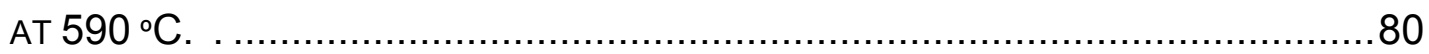

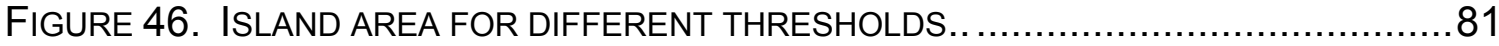

FIGURE 47 AREA OF THE CLUSTERS AS A FUNCTION OF TIME..................................83

FIGURE 48. LINE PROFILES FOR 3 DIFFERENT ANNEALING TEMPERATURES OF $535^{\circ} \mathrm{C}$,

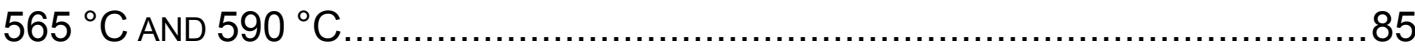

FIGURE 49. POWER SPECTRUM OF A COBALT FILM AT $535^{\circ} \mathrm{C}$ FOR DIFFERENT

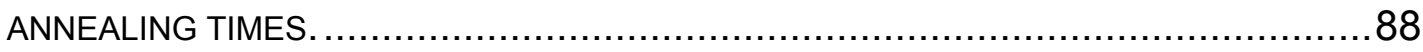

FIGURE 50. POWER SPECTRUM OF A COBALT FILM AT $565^{\circ} \mathrm{C}$ FOR DIFFERENT ANNEALING TIMES.

FIGURE 51. RADIAL AVERAGE OF THE POWER SPECTRUM FOR THE COBALT FILM ANNEALED AT $535^{\circ} \mathrm{C}$. 
FIGURE 52. RADIAL AVERAGE OF THE POWER SPECTRUM FOR THE COBALT FILM ANNEALED AT $565^{\circ} \mathrm{C}$.

FIGURE 53. AUTOCORRELATION FUNCTION FOR COBALT FILM ANNEALED AT $565^{\circ} \mathrm{C} . . .92$

FiguRE 54. AFM IMAGE OF A COBALT FILM ANNEALED AT $565^{\circ} \mathrm{C}$ DEPICTING THE CLUSTER WIDTH AND THE INTERCLUSTER SEPARATION...................................94

FIGURE 55. RADIAL AVERAGE OF THE HEIGHT-HEIGHT CORRELATION FUNCTION. . .....95

FIGURE 56. AVERAGE INTERCLUSTER SEPARATION AND CLUSTER WIDTH FOR A COBALT THIN FILM ANNEALED AT $535^{\circ} \mathrm{C}$ 95

FIGURE 58. OVERVIEW OF THE MAIN RESULTS FOUND ON COBALT DE-WETTING

EXPERIMENTS 97

FIGURE 59 SURFACE BARRIER ENERGIES FOR TWO NEIGHBOR ISLANDS.. 98

\section{INDEX OF TABLES}

TABLE 1. SURFACE ENERGY FOR FCC NI AND CU ON DIFFERENT CRYSTALLOGRAPHIC PLANES. ${ }^{26}$

TABLE 2. VARIATION OF THE EARLY WAVELENGTH WITH THE FILM THICKNESS FOR A LONG RANGE VAN DER WAALS INTERACTION.

TABLE 3. TyPICAL PARAMETERS FOR THE OMICRON VT NeEDLE SENSOR..................56

TABLE 4. FITTING PARAMETERS FOR ROUGHENING EXPERIMENTS. ..........................70

TABLE 5. FitTING PARAMETERS OF THE EXPONENTIAL CLUSTERS' GROWTH FOR 3

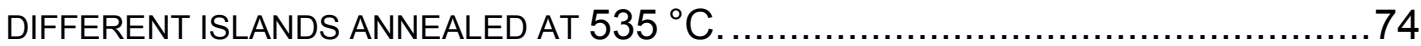

TABle 6. FitTING PARAMETERS OF A EXPONENTIAL FUNCTION TO THE CLUSTERS' HEIGHT AS A FUNCTION OF TIME.

TABLE 7. FITTING PARAMETERS FOR CLUSTER HEIGHT EVOLUTION FOR AN ANNEALING TEMPERATURE OF $535^{\circ} \mathrm{C}$.

TABLE 8. FITTING PARAMETERS FOR CLUSTER HEIGHT EVOLUTION FOR DIFFERENT ANNEALING TEMPERATURES. 


\section{INTRODUCTION}

Metal-oxide interfaces and coatings of metals on oxide substrates are very important in many technological and scientific areas. For medical applications, it is relevant to understand the adherence between metals and ceramics in order to produce high quality dental implants. ${ }^{1}$ For engineering, the understanding of metal-oxides interfaces is important for making good MOSFET devices ${ }^{1}$. On the other hand, in the emerging field of nanotechnology, it is also essential to understand how to control the size and distribution of metallic nano-clusters on oxide substrates and to determine their stability with time. Extensive investigations have been performed to characterize metal-oxide interfaces ${ }^{2}$, however, quantitative information on fundamental issues such as adhesion, interface energies and de-wetting mechanisms remains elusive.

For the applications described above, it is desirable to control the smoothness or roughness of a metallic film on an oxide substrate. In order to reach that goal it is necessary to answer the following question: what are the mechanisms allowing a metallic film to wet or de-wet an oxide substrate? In general, the behavior of oil, polymer or metal films on the surface of a substrate can be predicted by applying the Young-Dupre model to the system. ${ }^{3}$ This model predicts that if the surface tension (or surface energy) of the film-vacuum interface plus the surface energy of the film-substrate interface is larger that the surface energy of the substrate-vacuum interface, then the film will form drops in the surface, or in other words, it will de-wet. Unfortunately the surface tension energies of solid interfaces are not well known or poorly estimated, and hence the de-wetting of the film cannot be predicted using this model in most cases. Another approach is to analyze the surface dynamics of a thin film. In general, the surface dynamics are due to the interplay between surface tension of the metallic layer, which tends to smooth out the surface, and long range Casimir interactions across the film, which have a tendency to roughen it. This competition generates surface instabilities depending on variables such as temperature of annealing and 
thickness of the film. The instabilities generate corrugations or clusters in the film at early times. ${ }^{4}$ When the amplitude of these corrugations becomes comparable with the thickness of the metallic layer, the film will rupture and isolated islands will form in the substrate surface. It is well known ${ }^{4}$ that the separation between clusters $(\lambda)$ at early annealing times is proportional to $\Phi^{\prime \prime}(h)^{-1 / 2}$ where $\Phi "(h)$ is the second derivative of the long range Casimir interaction across the film and $h$ is its initial thickness. Experiments on thin films made from insulating materials, such as polymers deposited on silicon oxide, ${ }^{5}$ have proven that the long range interaction responsible of de-wetting is the common Van der Waals interaction $\Phi(h)=-A_{H} /\left(12 \pi h^{2}\right)$, where $h$ is the thickness of the film and $A_{H}$ is the Hamaker constant $\sim 7 \times 10^{-20} \mathrm{~J}$ for polymers. However the nature of the long range interaction leading to de-wetting of metallic thin films remains unclear. Experiments on cobalt thin films on sapphire carried out in this thesis will show that the intercluster separation leading to de-wetting would require a Van der Waals interaction with $A_{H} \sim 200 \times 10^{-20} \mathrm{~J}$, which is a completely unphysical result for metals. For conducting thin films it is proposed that the fermionic Casimir interaction (mediated by electrons) is responsible for initiating the de-wetting process, instead of the Van der Waals interaction more suitable for insulator films.

\section{DE-WETTING OVERVIEW}

De-wetting is the process where a thin film ruptures forming isolated clusters or drops (depending of the nature of the film) on top of the substrate. This process depends on the stability of the film after deposition. In general, thin films are in a metastable or unstable state after growth. These films might reach a stable condition by smoothing out the surface or forming islands on the substrate's surface, depending on the interplay of factors such as the nature of the interactions across the film, surface tension, interfacial energies and surface 
diffusion. Specifically, it is possible to distinguish two main processes leading thin films towards stable states (Figure 1): ripening and de-wetting.

In the ripening process, islands on top of deposited thin films tend to grow in size, making the surface smoother and more stable. It is well known that ripening is generated by three mechanisms acting alone or simultaneously (Figure 2): Ostwald ripening, surface diffusion and attractive island migration. In Ostwald ripening, islands are pinned on the substrate's surface. When these islands' sizes are smaller that a critical radius, they decrease their size until they disappear, but if they are larger than this critical radius, they grow (Figure 2(a)). With this process, clusters become larger and the result is a smoother surface. This phenomenon has been extensively studied in thin films composed of $\mathrm{Ag} / \mathrm{Ag}(111),{ }^{6,7} \mathrm{Ag} / \mathrm{Ag}(100),{ }^{8} \mathrm{Cu} / \mathrm{Cu}(001),{ }^{9} \mathrm{Si} / \mathrm{Si}(001),{ }^{10} \mathrm{Er} / \mathrm{Si}(001),{ }^{11} \mathrm{Mn} / \mathrm{Si}(001){ }^{12}$ and $\mathrm{TiN} / \mathrm{TiN}$ (111) (here the notation is film/substrate). ${ }^{13}$ An additional mechanism that occurs in the ripening process is diffusion. Atoms diffuse around islands edges creating the movement of the cluster's center of mass. The islands move randomly on the substrate producing the coalescence with neighboring cluster. This phenomenon has been observed experimentally in $\mathrm{Cu} / \mathrm{Cu}(100)$ and $\mathrm{Ag} / \mathrm{Ag}(100)$ islands. ${ }^{14}$ Another ripening mechanism observed in ripening is attractive island migration which was recently observed in $\mathrm{TiS}_{2} / \mathrm{Si}(111)$ clusters. ${ }^{15}$ In this case, islands of approximately the same size and closer to each other than some critical distance tend to move towards their center of mass, thus creating a larger island and producing a smoother film.

Ostwald ripening is the most common process in ripening and this mechanism has been studied theoretically for the general case of 3D/3D ( 3 dimensional clusters with diffusion in 3 dimensions) systems, ${ }^{16}$ and more recently, models has been adapted to the cases of $3 D / 2 D^{17}$ and $2 D / 2 D$ systems. ${ }^{18,19}$ In general, these models predict the dynamics of the ripening process, that is, how a cluster's size increases or decreases with time. The 3D/3D model shows that a 3D cluster with diffusion in 3D should grow with a power law $R \sim t^{a}$ where $R$ is the cluster's 
radius, $t$ is the time and $\alpha=1 / 3$. On the other hand, 3D/2D and 2D/2D models do not predict a power law in a straightforward way because of the dependence of the atoms' concentration on the surface of the film on some screening length. However mathematical approximations of the 3D/2D and 2D/2D models do predict a power law of the cluster's radius with time for small island density on the film's surface. In this case the islands' radius increases with a power law of $\alpha$ $=1 / 4$ for $3 \mathrm{D} / 2 \mathrm{D}$ and $\alpha=1 / 3$ for the $2 \mathrm{D} / 2 \mathrm{D}$ case .

\section{Surface Smoothing}

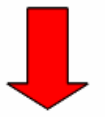

- Ostwald Ripening

- Attractive Island Migration

- Island Diffusion

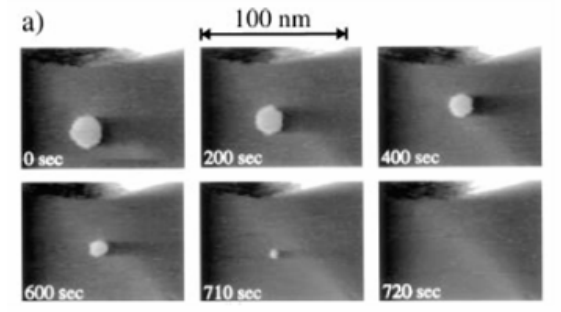

\section{Surface Roughening}

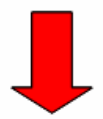

- Spinodal De-wetting

- Heterogeneous De-wetting

- Nucleation
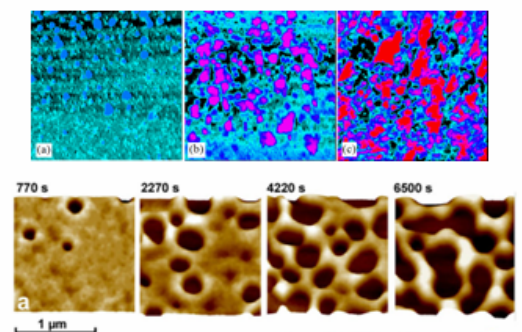

Figure 1 Processes thin films towards stable states. Images are experimental examples of the different processes. Images from left to right: $\mathrm{Ag} / \mathrm{Ag}(111) ;{ }^{6} \mathrm{Co}^{2} \mathrm{Al}_{2} \mathrm{O}_{3}(110) 1 \times 1 \mu \mathrm{m}^{2}$ (top); ${ }^{21}$ polystyrene/SiO ${ }_{2} 1.5 \times$ $1.5{\mu \mathrm{m}^{2}}^{2}$ (bottom). 
In roughening, and unlike ripening, surfaces become rougher due to the formation of pits or clusters. Roughening has been experimentally observed in $\mathrm{Co} / \mathrm{Al}_{2} \mathrm{O}_{3}(110),{ }^{21}$ above a critical temperature of $459^{\circ} \mathrm{C}$, and on $\mathrm{Cu} / \mathrm{MgO}(100)$ at

(a)

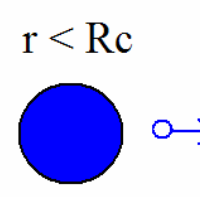

$\mathrm{r}>\mathrm{Rc}$

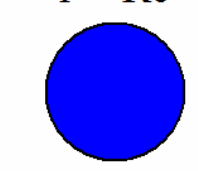

(b)
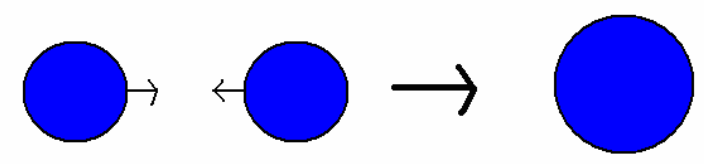

(c)
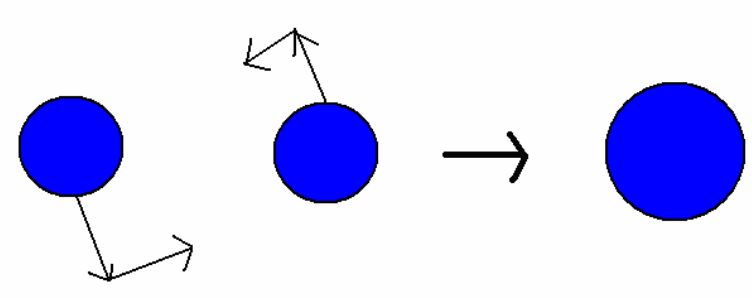

Figure 2. Clusters undergoing different ripening processes: (a) Ostwald ripening, (b) attractive island migration, and (c) diffusion.

$280{ }^{\circ} \mathrm{C} .{ }^{22}$ The morphology changes leading to the roughening of thin films belong to a more general process called de-wetting. In de-wetting, the films develop corrugations and dry patches on the surface of the substrate due to surface instabilities or inhomogeneities of the substrate. When these dry areas are large enough, the film forms droplets on the substrate and it is said that the film has completely de-wetted the substrate; hence the roughening of films must be understood only as an early stage of de-wetting. There are at least two types of de-wetting: spinodal and heterogeneous. In spinodal de-wetting, surface instabilities of the film generate height fluctuations of the film's thickness that increase exponentially with time. These height fluctuations create corrugations in the surface and eventually the film becomes very rough. When the amplitude of the height instabilities is comparable to the initial thickness of the film, the surface ruptures and holes are formed in the film. As time evolves, the holes grow and coalesce with each other producing larger de-wetted areas in the film. The late 
stage of de-wetting is the formation of drops or isolated clusters on the substrate. Experimental evidence of spinodal de-wetting has been found on liquid metal films ${ }^{23}$ and polystyrene on silicon substrates. ${ }^{5}$ In heterogeneous de-wetting, impurities in the substrate underneath the film or inhomogenities in the oxide layer of the substrate or substrate's step edges might produce nucleation of holes leading to de-wetting. Heterogeneous de-wetting has been observed in polystyrene films ${ }^{4}$ and in ultrathin silver films on $\mathrm{Si}(111) .{ }^{24}$

\section{MOTIVATION FOR STUDING COBALT THIN FILMS}

A few years ago, annealing experiments ${ }^{25}$ were carried out in cobalt thin films sputtered on sapphire (110). Films annealed at $550^{\circ} \mathrm{C}$ and quenched to room temperature showed the formation of rectangular pits (see Figure 3 ) with sizes of approximately $300 \mathrm{~nm}$. The pit formation coincided with a reentrant smoothing of the film and the change of the cobalt crystal structure from fcc (100) to fcc (111). It was not clear in this experiment what was the mechanism for the formation of the pits nor how the rapid quenching might have affected the morphology of the film. In order to avoid the effect of film quenching, in-situ experiments ${ }^{21}$ were carried out on cobalt films evaporated using metal e-beam epitaxy (MBE) on sapphire (110). The films were annealed and simultaneously imaged (Figure 4) in a range of temperatures between $20^{\circ} \mathrm{C}$ and $600^{\circ} \mathrm{C}$. The roughness of the film defined as $\rho=\frac{1}{M N} \sqrt{\sum_{i}^{M} \sum_{j}^{N}\left(z_{i, j}-\bar{z}\right)^{2}}$ (where $M$ and $N$ are the image's size, $z_{i, j}$ the image's height at the pixel position $(i, j)$ and $\bar{z}$ the average image's height) were plotted as a function of temperature and it was found to depend on the temperature as $\rho(T) \propto\left(\left(T / T_{0}\right)-1\right)^{\alpha}$, where $\rho$ is the roughness of the film, $T$ the temperature, $T_{0}$ a critical temperature and $\alpha$ a critical exponent. It was found that $\alpha=0.5$ and $T_{0}=459^{\circ} \mathrm{C}$. Unlike previous experiments, there was no evidence of 
pit formation at $550{ }^{\circ} \mathrm{C}$ and only very irregular ones were observed at temperatures closer to $600{ }^{\circ} \mathrm{C}$. Another observation from this experiment was that the roughness had a very strong dependence on the annealing time; hence experiments of this kind should control not only the annealing temperature but also the annealing history of the films.

Considering these experiments, the most important questions that need to be addressed are: What is the mechanism that initiates the cluster or pit formation in the cobalt films? and what is the mechanism responsible of the clusters' evolution? This thesis will attempt to answer these questions by studying the morphology of cobalt thin films deposited on sapphire (110) annealed between $535{ }^{\circ} \mathrm{C}$ and $590{ }^{\circ} \mathrm{C}$ as a function of time. These experiments will yield clues

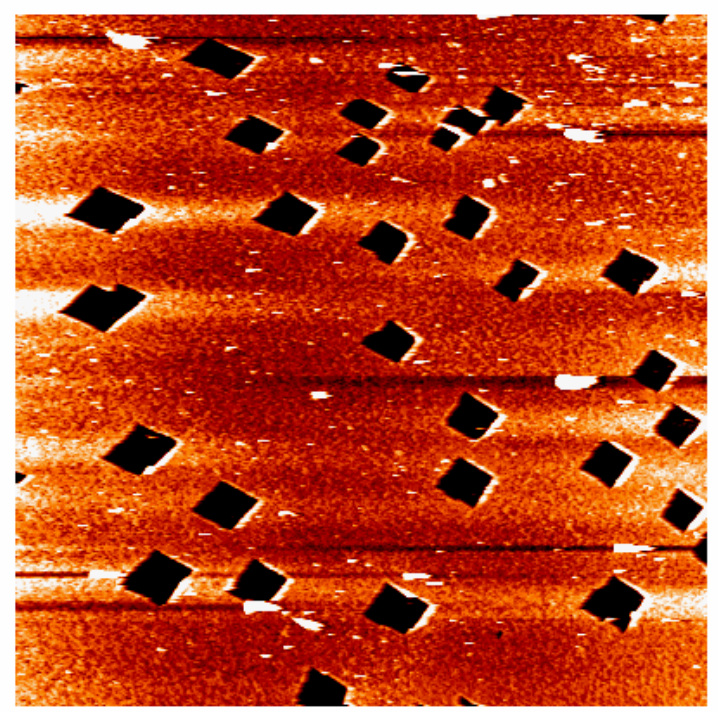

Figure 3. Ex-situ AFM image ${ }^{25}$ of a cobalt thin film ( $5 \mathrm{~nm}$ thick) on sapphire (110) annealed at $550^{\circ} \mathrm{C}$. Image size $5 \times 5 \mu \mathrm{m}^{2}$. After Ref. [25].

about the physical mechanisms leading to the formation of clusters in the annealed thin films. The next sections of Chapter 1 contains an introduction to the theoretical models of ripening in 3D/3D, 3D/2D and $2 D / 2 D$ and the basic mechanisms responsible de-wetting. Chapter 2 will focus on the experimental 
procedures, Chapter 3 will show the main results of the experiments and finally Chapter 4 will discus the results based on the theories presented in this chapter.
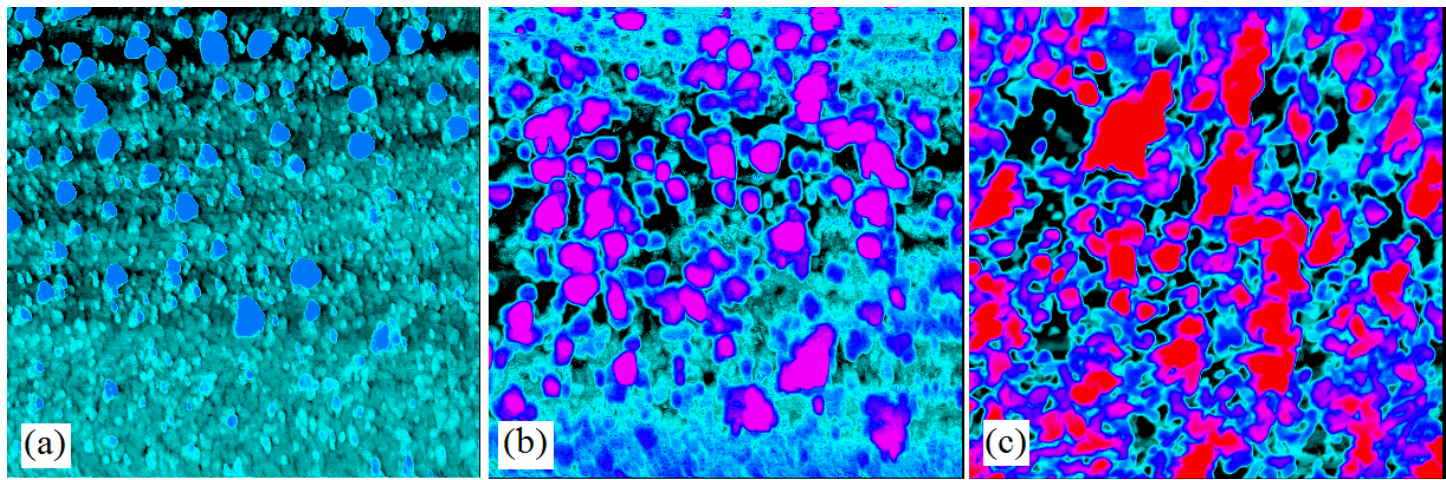

Figure 4. AFM images of a $5 \mathrm{~nm}$ cobalt thin film on sapphire (110) annealed at different temperatures. Snapshots show the same section of the film annealed at (a) $\mathrm{T}=462{ }^{\circ} \mathrm{C}$, (b) $\mathrm{T}=482{ }^{\circ} \mathrm{C}$ and (c) $\mathrm{T}=524{ }^{\circ} \mathrm{C}$. Size of the images: $1 \times 1 \mu \mathrm{m}^{2}$. After Ref. [21].

\section{BASIC CONCEPTS}

Prior to introducing the phenomena of cluster roughening and de-wetting, it is necessary to explain basic concepts such as surface energy and atomic diffusion in surfaces.

\subsection{SURFACE ENERGY}

The surface energy (or surface tension) is the amount of energy necessary to generate one unit of surface area. In order to create an extra surface, it is necessary to destroy the bonds of the atoms located at the surface/vacuum interface, thus exposing the atoms of the bulk; evidently this process requires some energy. Surface energy is important because it determines the equilibrium 
shape of crystals and plays an important role in de-wetting and crystal growth. Figure 5 shows the process of area formation for a monoatomic solid. The solid in state 1 is surrounded by a layer of atoms covering a certain area depicted in blue. The solid in state 2 creates a unit of extra area $A$ in green. For this representation and assuming mass conservation, the surface energy can be expressed as

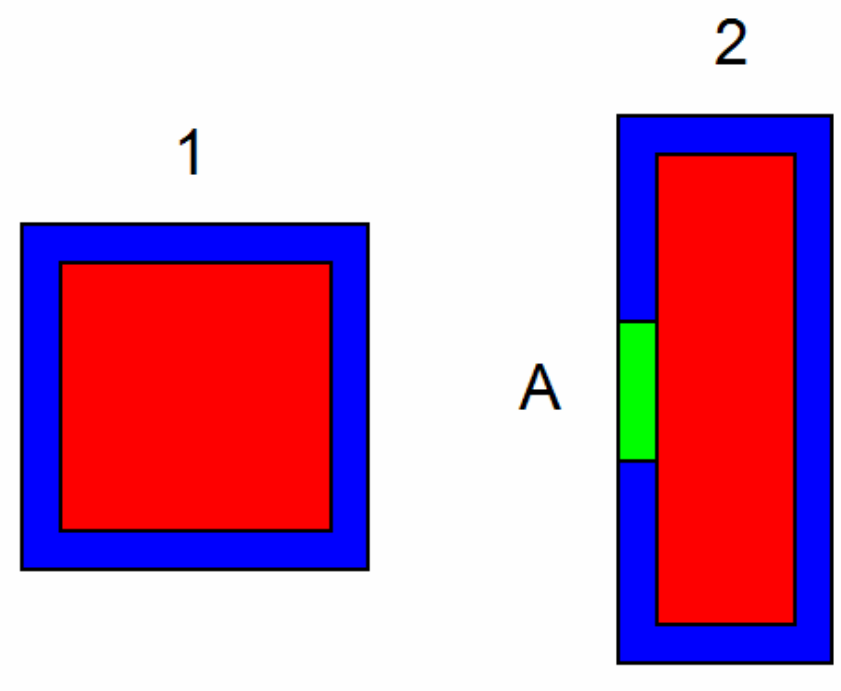

Figure 5. The figure depicts the formation of a unit of area $A$ for a monoatomic solid.

$$
\gamma=\frac{1}{A}\left(E_{t o t}^{2}-E_{t o t}^{1}\right),
$$

where $E_{\text {tot }}^{1}$ is the total energy for the configuration 1 and $E_{\text {tot }}^{2}$ is the total energy for the configuration 2. In general it is very difficult to measure surface energies; however with the ever-increasing computer speeds, it is possible nowadays to calculate the energies from first principles quickly and in a reliable manner. Figure 6 shows the surface energy computed for several $3 d$ transition metals. ${ }^{26}$ 
From the figure it is clear that the surface energy increases when the d-band is half occupied. This result is related to the number of broken bonds on the surface; for half-full d-bands, the number of surface broken bonds is higher than the number of broken bonds for almost full or almost empty bands. Hence the surface energy increases for $\mathrm{V}, \mathrm{Cr}$ and $\mathrm{Mn}$.

A liquid or an amorphous material can minimize its surface energy by decreasing the area exposed to vacuum, but this is not necessarily true for crystals. Anisotropy plays an important role in surface energy, determining the final shape of a crystal and in most cases their equilibrium shapes may be a very complex polyhedron. Experimentally this can be observed when a crystal is cleaved along different orientations. For some directions the crystal might break apart very easily but for others it might require a significant amount of energy. In general, cleaving is easier along planes where the surface energy is smaller or on other words in planes where atoms in the surface are less coordinates. This concept can be seen through the Friedel model ${ }^{27}$ in which the surface energy $\gamma \propto\left(1-\sqrt{z_{s} / z_{b}}\right)$, where $z_{s}$ is the coordination number of an atom located in the surface and $z_{b}$ the coordination number of an atom in the bulk. Table 1 shows the surface energy for fcc $\mathrm{Ni}$ and $\mathrm{Cu}$ along different orientations. The surface energy is $1.952 \mathrm{~J} / \mathrm{m}^{2}$ for the $\mathrm{Cu}(111)$ plane, $2.237 \mathrm{~J} / \mathrm{m}^{2}$ for the (110) plane and $2.166 \mathrm{~J} / \mathrm{m}^{2}$ for the $(100)$ plane. The difference in energy results from ratio $\mathrm{z}_{\mathrm{s}} / \mathrm{z}_{\mathrm{b}}$. For example the number of nearest neighbors for an atom in the bulk of a fcc lattice is 12. An atom in the same lattice located on the surface of a (111) plane has 9 nearest neighbors $\left(z_{s} / z_{b}=9 / 12\right)$ and 3 broken bonds per atom at the surface. Similarly, for atoms on the surface of a fcc (110) plane $z_{s} / z_{b}=7 / 12$ (5 broken bonds per atom) and for atoms on the fcc (110) plane $z_{s} / z_{b}=8 / 12$ (4 broken bonds per atom). Hence using the Friedel model the surface energy goes as: $f c c(111)<f c c(100)<f c c(110)$. For Ni the energy for the $f c c(111)$ is the smallest, which agrees well with Friedel model. However the surface energy for the fcc(110) plane is slightly smaller than the energy for the fcc(100) plane. This difference is due to the fact that the Friedel model is not perfect and other 
important phenomena modify the final surface energy of crystals, such as surface reconstruction, surface relaxation and the polar or non-polar character of a given surface plane.

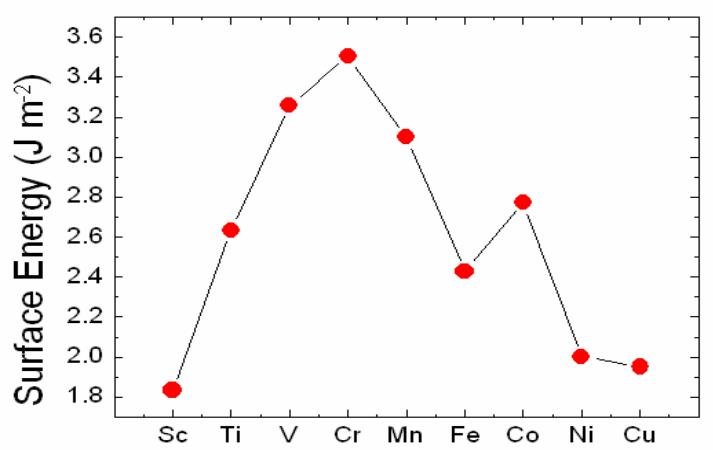

Figure 6. Surface energy for several 3d transition metals. Data taken from Ref. [26].

\begin{tabular}{c|c|c}
\hline Plane & $\begin{array}{c}\mathrm{Cu} \\
\gamma\left(\mathrm{J} / \mathrm{m}^{2}\right)\end{array}$ & $\begin{array}{c}\mathrm{Ni} \\
\gamma\left(\mathrm{J} / \mathrm{m}^{2}\right)\end{array}$ \\
\hline$(111)$ & 1.952 & 2.011 \\
$(100)$ & 2.166 & 2.426 \\
$(110)$ & 2.237 & 2.368 \\
\hline
\end{tabular}

Table 1. Surface energy for fcc $\mathrm{Ni}$ and $\mathrm{Cu}$ on different crystallographic planes. $^{26}$

Taking into account the anisotropy of the surface energy for crystals, their final shape can be calculated from the following expression: ${ }^{28}$

$$
\int \gamma(h k l) d A=\text { minimum, }
$$

where $\gamma(h k l)$ is the surface energy of the atoms in the (hkl) plane and $d A$ is an element of area of the crystal. In order to determine the minimum energy configuration of a crystal, the Wulff construction plays an important role. Wulff plots are built by drawing $\gamma(h k l)$ in polar coordinates. Here $\gamma$ is the magnitude of the radial vector proportional to the surface energy of an $(h k l)$ plane and the polar angle $\theta$ is the angle of the direction $<h k l>$. The final Wulff structure is given by 
the intersection of different planes perpendicular to the radial vectors $\gamma(\theta)$. Figure 7 shows a Wulff construction for bcc Fe. Here $\gamma(010)$ is the radial vector proportional to a surface energy of $2.222 \mathrm{~J} / \mathrm{m}^{2}$ and $\gamma(110)$ the radius of a vector proportional to $2.430 \mathrm{~J} / \mathrm{m}^{2}$ (energies extracted from Ref. 26). It is evident from the sketch that the (110) plane (with the highest surface energy) is the one least exposed to vacuum because it minimize the total surface energy of the crystal.

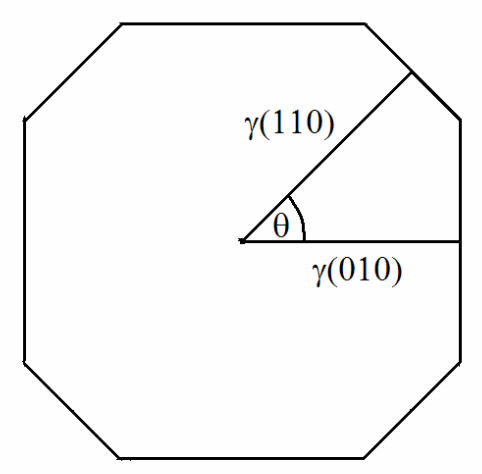

Figure 7. Wulff construction for bcc Fe in the (001) plane.

\subsection{DIFFUSSION}

Diffusion is a kinetic process that generates the homogenization of chemical components in a single phase. In solids, diffusion occurs on the atomic scale but as time increases the length scale over which the uniformity takes place might extend to macroscopic distances. The first observations of diffusion were carried out in 1855 by A. Fick in a water-salt system; his experiments allowed him to derive phenomenological laws of diffusion and to introduce for the first time the concept of diffusivity. 
Fick's first phenomenological law states that the magnitude of the mass flux is proportional to the gradient of the concentration at that point, so that

$$
\vec{J}=-D \vec{\nabla} C,
$$

where $\vec{J}$ is the mass flux per unit of area and time, ${ }^{i} D$ is the diffusivity in units of $\mathrm{m}^{2} \mathrm{~s}^{-1}$ and $C$ the concentration in $\mathrm{kg} \mathrm{m}^{-3}$. This law states that the mass flux will flow from high concentration points to low concentration points. The particular characteristics of the system as well as the temperature dependence on the mass flux are hidden within the diffusivity constant.

If the system under study does not have sinks or sources of material, mass is conserved and therefore the difference between the inflow and outflow of mass in a given volume of space $d V$ must be equal to the change of the concentration in that region of space. This is expressed by

$$
\frac{\partial C}{\partial t}=-\vec{\nabla} \cdot \vec{J}
$$

If $\vec{\nabla} \cdot \vec{J}<0$ there is a converging flow at that point and the concentration increases with time. On the other hand, if $\vec{\nabla} \cdot \vec{J}>0$ there is a divergent flow at that point and the concentration will decrease.

It is possible to combine Fick's first law with the mass conservation expression as

$$
-\vec{\nabla} \cdot(-D \vec{\nabla} C)=\frac{\partial C}{\partial t}
$$

or

$$
\nabla^{2} C=\frac{1}{D} \frac{\partial C}{\partial t}
$$

\footnotetext{
'Note that these units correspond to a system with diffusion in 3D. In the case of a system with diffusion in 2D, the mass flux has units of $\mathrm{Kg} \mathrm{m}^{-1} \mathrm{~s}^{-1}$ and the concentration units of $\mathrm{Kg} \mathrm{m}^{-2}$.
} 
Equation (6) is the so-called Fick's second law and in general it is used to calculate the appropriate form of the concentration field for a given geometry and boundary conditions of a system.

\section{CLUSTER RIPENING}

\subsection{D/3D MODEL FOR RIPENING}

The ripening problem for 3D/3D ( 3 dimensional clusters with diffusion in 3 dimensions) was studied by Lifshitz and Slyzov many years ago in what is called the LS theory. ${ }^{29}$ Later on, the ripening problem was extended to $2 \mathrm{D} / 2 \mathrm{D}$ by Marqusee $^{30}$ and to $3 \mathrm{D} / 2 \mathrm{D}$ by Sorolin ${ }^{31}$. The LS theory supposes the existence of a distribution of spherical droplets in a minority phase immersed in a medium with some concentration of material in the majority phase. Fick's first law states that the flow of material in and out of the droplet will depend on the gradient of the concentration field as

$$
\vec{J}=-D \vec{\nabla} C,
$$

where $\vec{J}$ is the mass flow current, $D$ the diffusion and $C$ the concentration field. In order to determine C, Fick's second law (Equation 6) must be solved. Supposing a steady state and considering spherical coordinates, Fick's second law becomes

$$
\nabla^{2} C=\frac{1}{r^{2}} \frac{\partial}{\partial r}\left(r^{2} \frac{\partial C}{\partial r}\right)=0
$$

where $r$ is the curvature radius measured from the center of the droplet. Solving the equation for $C$ results in 


$$
C(r)=-\frac{A}{r}+B .
$$

Note that for large $r$,

$$
\lim _{r \rightarrow \infty} C(r)=B .
$$

This is an interesting result because the steady state solution of Fick's second law in $3 D$ is finite when $r$ goes to infinity. The constants $A$ and $B$ can be determined in the limit where $r=R$, where $R$ is the radius of the droplet and $C(R)$ is the concentration at the droplet edge. For $r=$ infinity (i.e. for a flat surface) a constant concentration field $\bar{C}$ is assumed, so that

$$
C(r=R)=C(R)=-\frac{A}{R}+B,
$$

and therefore

$$
C(r=\infty)=\bar{C}=B .
$$

This leads to

$$
C(R)=-\frac{A}{R}+\bar{C},
$$

hence

$$
C(r)=\bar{C}+[C(R)-\bar{C}] \frac{R}{r} .
$$

The last equation is the concentration field as a function of the distance from the cluster's center for a 3D droplet. Then, the total mass flowing into the droplet per unit of time is given by:

$$
I=4 \pi R^{2} D|\vec{\nabla} C|_{R} \mid
$$

or 


$$
I=4 \pi R^{2} D(\bar{C}-C(R)) .
$$

The mass flowing into the droplet will produce a variation in the volume of the cluster with time

$$
I=\frac{d}{d t}\left(\frac{4}{3} \pi R^{3} \rho\right)=4 \pi \rho R^{2} \dot{R},
$$

where $\rho$ is the density of the cluster's material $\left(\mathrm{Kg} \mathrm{m}^{-3}\right)$. The last equation assumes that the drops are isotropic. Finally, equating relationships (16) and (17) results in

$$
\dot{R}=\frac{D}{R \rho}(\bar{C}-C(R)) .
$$

Equation (18) is the central result of the LS theory. If the concentration of the medium is greater than the concentration at the droplet edge, the cluster will grow with time, but if the concentration of the medium is smaller than the cluster's edge, the droplet or cluster will become smaller and eventually it will disappear; this result is called Ostwald ripening. To determine the behavior of the cluster's radius with time, the radial dependence of the cluster concentration must be known. Gibbs and Thomson determined the form of $C(R)$ in what is called the GT equation: ${ }^{32}$

$$
C(R)=\bar{C} \exp \left(\frac{\gamma \Omega}{R k T}\right),
$$

where $\gamma$ is the surface energy of the droplet, $\Omega$ is the volume per atomic unit, $k$ is Boltzmann's constant, $R$ is the curvature radius of the cluster and $T$ is the temperature. This equation predicts that small clusters (small curvature radius) will have large concentration at the surface. With the assumption that $\gamma \Omega<<R k T$, Equation (19) and that $\bar{C}>C(R)$ (cluster's growth): 


$$
C(R) \approx \bar{C}\left(1+\frac{\gamma \Omega}{R k T}\right)
$$

Plugging Equation (20) into (18) yields

$$
R^{2} \dot{R} \approx \text { const, }
$$

and this leads to a dependence of the cluster radius with time

$$
R \propto t^{\frac{1}{3}} .
$$

The last equation predicts a power law of cluster's growth with an exponent of 1/3. Of course if $\bar{C}<C(R)$ then $R \propto-t^{1 / 3}$ and the cluster shrinks with time. This exponent was determined under the assumption of diffusion in 3D for a 3D cluster, but as will be demonstrated in the next section, this power law also applies to 2D/2D systems under special circumstances.

\subsection{D/2D MODEL FOR RIPENING}

The ripening problem for 2 dimensional clusters with diffusion in 2 dimensions is more challenging because the concentration field diverges when $r$ goes to infinity as can be seen by solving the steady state Fick's second law in polar coordinates,

$$
\nabla^{2} C=\frac{1}{r} \frac{\partial}{\partial r}\left(r \frac{\partial C}{\partial r}\right)=0
$$

so that

$$
C(r)=A+\ln r .
$$

To overcome this problem, Marquse ${ }^{30}$ proposed a modification of Fick's second law (Equation 6) including a source and a sink term for the concentration field. The modified equation is 


$$
\frac{\partial C}{\partial t}=D \nabla^{2} C-D \xi^{-2} C+S
$$

where $D \xi^{-2} C$ is a sink term with units of $\mathrm{Kg} \mathrm{m}^{-2} \mathrm{~s}^{-1}, \xi$ is a screening length in units of meters and $S$ is a source term. In a steady state the source and the sink terms have to be equal, so that

$$
\frac{\partial C}{\partial t}=0 \Rightarrow S=D \xi^{-2} \bar{C},
$$

and therefore Equation (25) can be written as

$$
\left\lfloor\nabla^{2}-\xi^{-2}\right\rfloor \delta C=0
$$

where $\delta C=C(R)-\bar{C}$. The solution to this equation is given by

$$
C(r)=\bar{C}+[C(R)-\bar{C}] \frac{K_{1}(r / \xi)}{K_{0}(R / \xi)},
$$

where $K_{0}$ and $K_{1}$ are the zeroth and first order modified Bessel functions, respectively. Plugging the concentration field given by the last equation into Fick's first law yields

$$
I=\left.2 \pi R D \nabla C\right|_{R},
$$

where

$$
I=\frac{2 \pi D R}{\xi}[C(R)-\bar{C}] \frac{K_{1}(R / \xi)}{K_{0}(R / \xi)}
$$

The mass flux in and out of the droplet will be equal to the change of mass in the $2 \mathrm{D}$ cluster such that

$$
I=\frac{d}{d t}\left(\rho \pi R^{2}\right)=2 \pi \rho R \dot{R},
$$


where $\rho$ is the mass density of the cluster with units of $\mathrm{Kg} \mathrm{m}^{-2}$. Equating (30) and (31) yields

$$
\dot{R}=\frac{D}{\rho \xi} \frac{K_{1}(R / \xi)}{K_{0}(R / \xi)}[\bar{C}-C(R)]
$$

In this case, the growth law does not lead to a power law because of the presence of the modified Bessel function. Also, the growth depends on the surrounding medium through the screening length, which in turn might depend on time. However, if $R<<$,

$$
K_{0}(R / \xi) \approx \ln \left(\frac{\xi}{R}\right)
$$

and

$$
K_{1} \approx \frac{\xi}{R}
$$

Replacing these approximations into equation (32) results in

$$
\dot{R}=\frac{D}{R \rho \ln (\xi / R)}[\bar{C}-C(r)]
$$

Studies show ${ }^{30,33}$ that there is only a weak dependence of the $\ln (\xi / R)$ term on $R$, which is the same as considering a screen length of the form

$$
\xi=(\lambda-1) R,
$$

where $\lambda$ is a constant. Using this expression for the screen length, the GT equation and $\bar{C}>C(r)$ in Equation (35) then

$$
R^{2} \dot{R}=\text { const } \Rightarrow R \propto t^{1 / 3}
$$


In this situation, a power law of $1 / 3$ results as in the 3D/3D model. In general for a 2D/2D system, a power law cluster growth behavior is not valid because of the dependence of the concentration field on the screening length. On the other hand, a power law can be deduced if the screening length is much larger than the radius of the cluster (i.e. low density of clusters per unit area) and if there is a weak dependence of the screen length on $R$.

a)
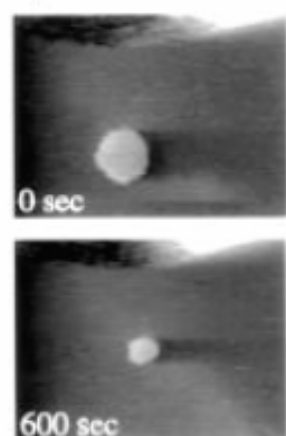

$100 \mathrm{~nm}$
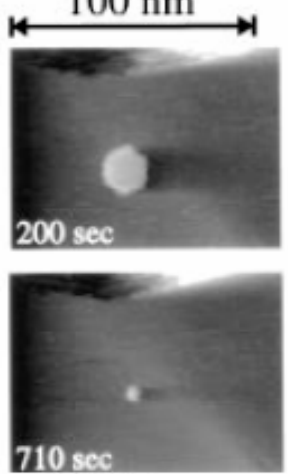
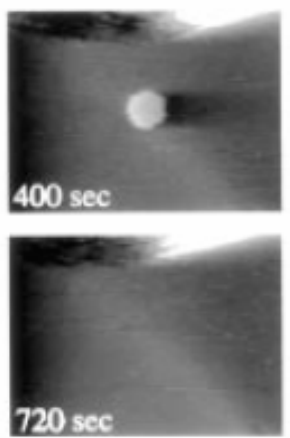

Figure 8. STM image showing the decay of a 2D Ag cluster on Ag (111) at room temperature. After Ref. [6].

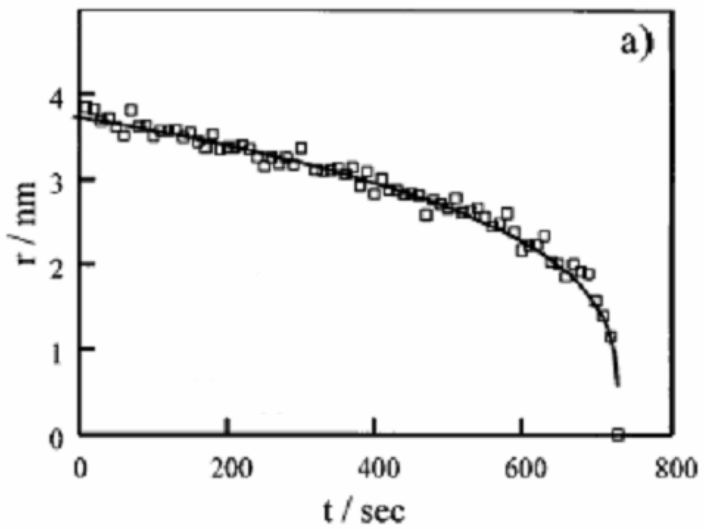

Figure 9. Decay of the radius for a 2D Ag island on Ag (111). After Ref. [6]. 
Experimental evidence for 2D/2D ripening is shown in Figures 8 and 9. Figure 8 shows a 2 dimensional cluster of $\mathrm{Ag}$ on top of $\mathrm{Ag}(111)$ at room temperature. ${ }^{6}$ The cluster is placed at about $50 \mathrm{~nm}$ from a substrate step (top edge on the images). In the snapshots it is clear that the cluster becomes smaller with time as predicted by the ripening process disappearing completely after 720 seconds. Figure 9 shows the evolution of the cluster's radius; the solid line is the fitting of the radius with time using a power exponent of $1 / 3$, this exponent is consistent with 2D island decay due to diffusion in 2D.

\subsection{D/2D MODEL FOR RIPENING}

The case of 3-dimensional clusters with diffusion on a 2D surface has the same problem as the $2 \mathrm{D} / 2 \mathrm{D}$ system because of the divergence of the concentration field at long distances. Here it also is necessary to apply a source and a sink to balance the concentration equation when $r=$ infinity. Applying the same ideas used in the $2 \mathrm{D} / 2 \mathrm{D}$ case,

$$
I=\frac{2 \pi D R}{\xi}[\bar{C}-C(R)] \frac{K_{1}(R / \xi)}{K_{0}(R / \xi)} .
$$

Equation (38) has the same form as Equation (30), but differences appear when considering the mass rate entering a spherical cluster with radius $R$ (same as the 3D/3D case), where

$$
I=\frac{d}{d t}\left(\frac{4}{3} \pi R^{3} \rho\right)=4 \pi \rho R^{2} \dot{R} .
$$

Equating (38) and (39) results in

$$
\dot{R}=\frac{D}{2 \rho \xi R} \frac{K_{1}(R / \xi)}{K_{0}(R / \xi)}[\bar{C}-C(R)] .
$$


The growth rate for $3 \mathrm{D} / 2 \mathrm{D}$ also does not follow a power law similar to the $2 \mathrm{D} / 2 \mathrm{D}$ case because of the modified Bessel function. Considering again $R \ll \xi$ yields

$$
\dot{R}=\frac{D}{2 \rho R^{2}} \frac{1}{\ln (\xi / R)}[C(R)-\bar{C}] .
$$

Using the condition for the screen length in Equation (37), the GT equation and the condition $\bar{C}>C(R)$ results in

$$
R^{3} \dot{R}=\text { const } \Rightarrow R \propto t^{1 / 4} .
$$

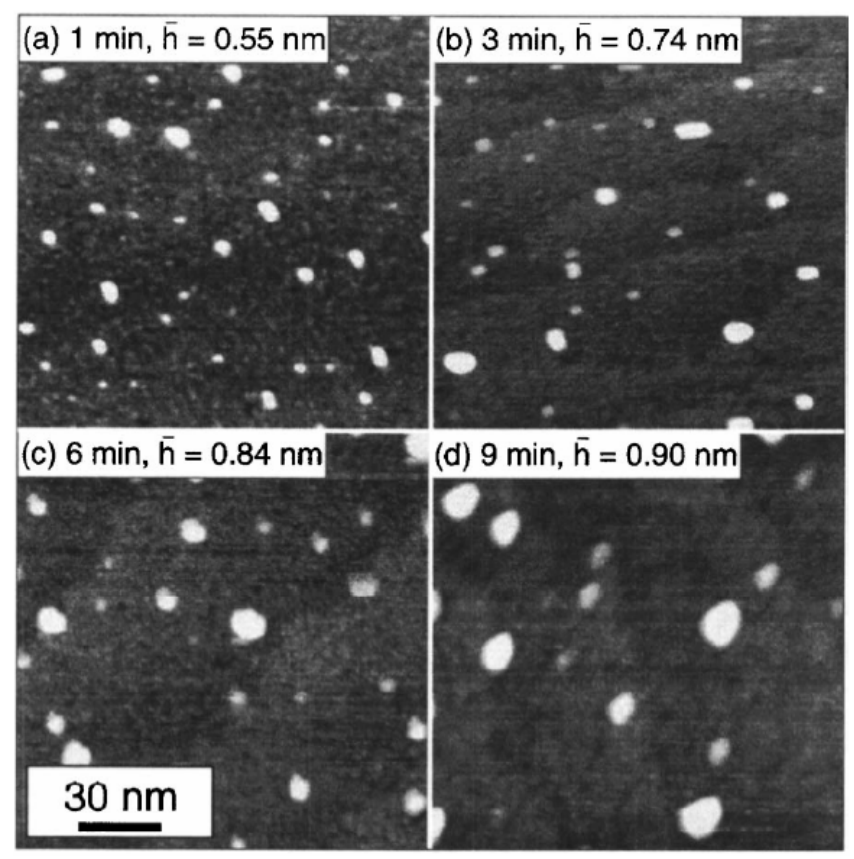

Figure 10. STM images of $\mathrm{Mg}$ silicide islands on Si (001) annealed at 500 ${ }^{\circ} \mathrm{C}$ for several minutes. After Ref. [12].

Therefore, a power law of $1 / 4$ is expected for a 3 -dimensional cluster with diffusion in 2D only under special circumstances. In general no power law should be expected due to the dependence of the growth equation with the screening 
length. An example of ripening in 3D/2D can be observed in Figure 10, where a $\mathrm{Mg}$ thin film $(0.1 \mathrm{ML})$ forms isolated clusters on $\mathrm{Si}(001)$ after annealing at $500{ }^{\circ} \mathrm{C}$ for several minutes. ${ }^{34}$ The number of clusters decreases with time while their diameter increases phenomena which are described by the ripening theory. On the other hand, the average height of the clusters (Figure 11) increases with time following a power law with an exponent of 0.25 . The low coverage of $\mathrm{Mg}$ might have produced a screening length that was much larger than the radius of the clusters leading to the theoretical (3D/2D) power law growth of $H \sim t^{1 / 4}$.

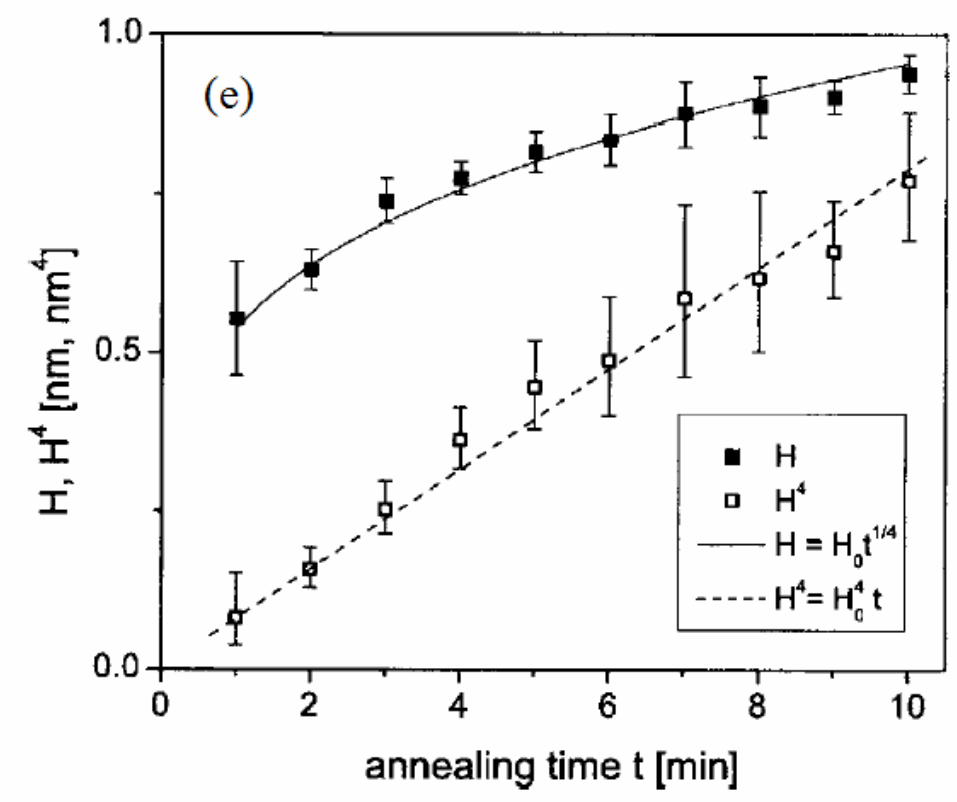

Figure 11. Average cluster's height $\mathrm{H}$ as a function of time for $\mathrm{Mg}$ silicide islands on $\mathrm{Si}(001)$. The dashed line shows the linear fitting of $\mathrm{H}^{4}$ versus time. After Ref. [12]. 


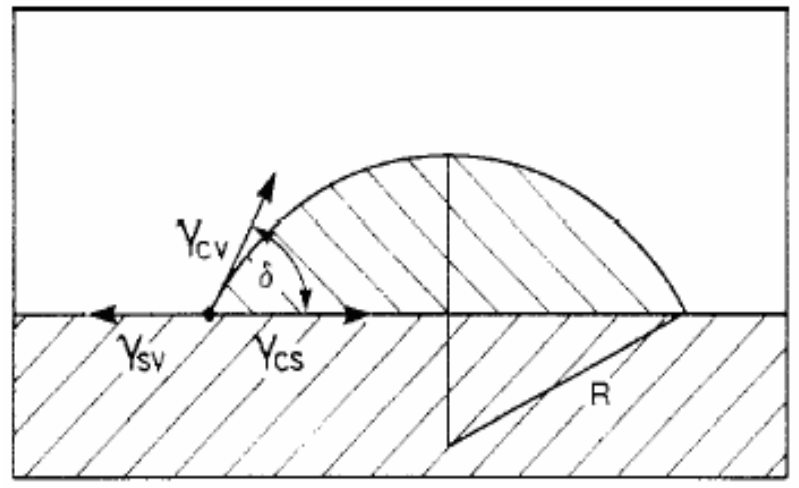

Figure 12. Sketch of a drop on top of a surface depicting the YoungDupre model of dewetting. $\gamma_{C V}, \gamma_{S V}$ and $\gamma_{C S}$ are interface energies at the cluster/vacuum, surface/vacuum, and cluster/surface interfaces, respectively; $\delta$ is the angle between the drop and the substrate and $R$ is the radius of curvature of the drop (after Ref. [3]). 


\section{DEWETTING}

De-wetting is the property of solids or liquids to form clusters or drops on top of substrates. A classic model for de-wetting is the Young-Dupre equation ${ }^{3}$

$$
\gamma_{S V}=\gamma_{C S}+\gamma_{C V} \cos \delta,
$$

where $\gamma_{S V}$ is the surface energy of the substrate/vacuum interface, $\gamma_{C S}$ is the surface energy of the cluster/substrate interface and $\gamma_{C V}$ is the surface energy of the cluster/vacuum interface (Figure 12). This formula predicts a complete

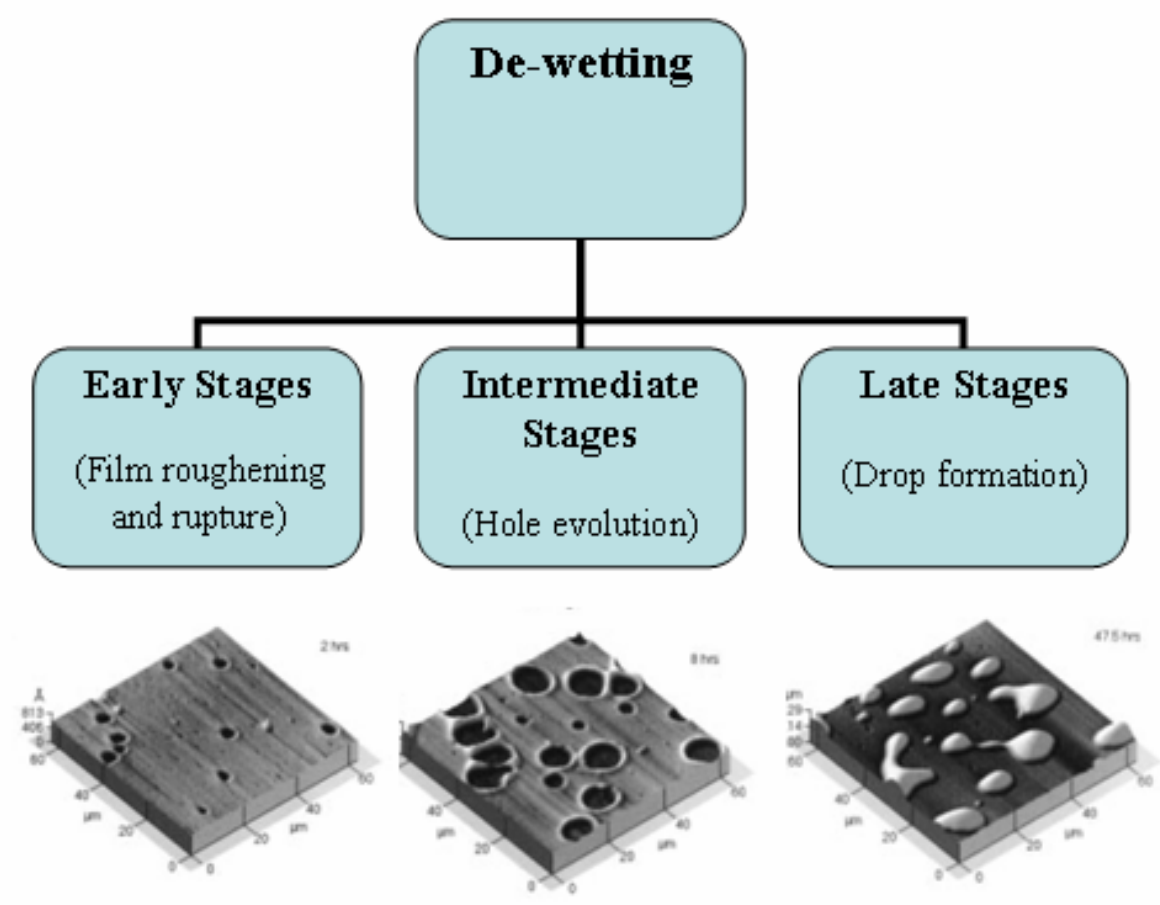

Figure 13. De-wetting evolution with time. Bottom images correspond to a diblock copolymer $45 \mathrm{~nm}$ thick on Si annealed at $170{ }^{\circ} \mathrm{C}$. Annealing times from left to right: $2 \mathrm{hr}, 12 \mathrm{hr}$ and $48 \mathrm{hr}$. Image sizes: $60 \times 60 \mu \mathrm{m}$. After Ref. [35].

wetting of the surface for $\delta=0$. For $\delta>0$ the film should break in droplets; therefore de-wetting occurs when $\gamma_{C V}+\gamma_{C S}-\gamma_{S V}>0$. 
Unfortunately, the interfacial energies are in general unknown or poorly estimated; hence de-wetting can not be predicted accurately. Another problem is that the Young-Dupre equation works only for liquids but fails for solid crystals on solid interfaces because the rounded contact shapes are replaced by anisotropic crystallographic facets. ${ }^{36}$

Another approach is to analyze surface dynamic processes by studying the evolution of clusters and pits as a function of time. This procedure separates the de-wetting process into 3 stages (Figure 13). In the early stage, the concern is to analyze how the films become rough and how this roughening produces holes in the film. In the intermediate stage, the evolution of holes as a function of time is analyzed. Finally, in the late stage the coalescence of holes with each other is studied, which eventually produces a rupture of the film and the formation of drops on top of the substrate.

\subsection{EARLY STAGE OF DE-WETTING}

The aim of this thesis is to understand the roughening and de-wetting phenomena of cobalt films on sapphire, and therefore most of our attention will be devoted to understanding the early stages of de-wetting rather than the intermediate and late stages. Early stages of de-wetting have been extensively studied in polymers, oils and liquid, and therefore the first part of this section will be devoted to presenting the basic theories and concepts of de-wetting applicable to liquids, while the second part will explain the kinetic theory of dewetting applicable to solids.

\subsubsection{De-wetting in fluids}

There are 2 main processes in triggering dewetting: spinodal and heterogeneous de-wetting. 
Spinodal dewetting

In thin films there are 2 main interactions in the surface: capillarity and long range interactions. Capillarity or surface tension makes the film flat and smooth whereas long range interactions (like Van der Waals forces) have a tendency to thicken the film. The interplay of these interactions determines the stability of the film. Unstable or metastable films might develop surface corrugations that grow exponentially with time, eventually producing the rupture of the film. Vrij and Overbeek ${ }^{37}$ were the first to analyze this problem theoretically using a mathematical analogy between spinodal decomposition of a fluid mixture (hence the name given to the spinodal de-wetting), where the fluctuations in the fluid

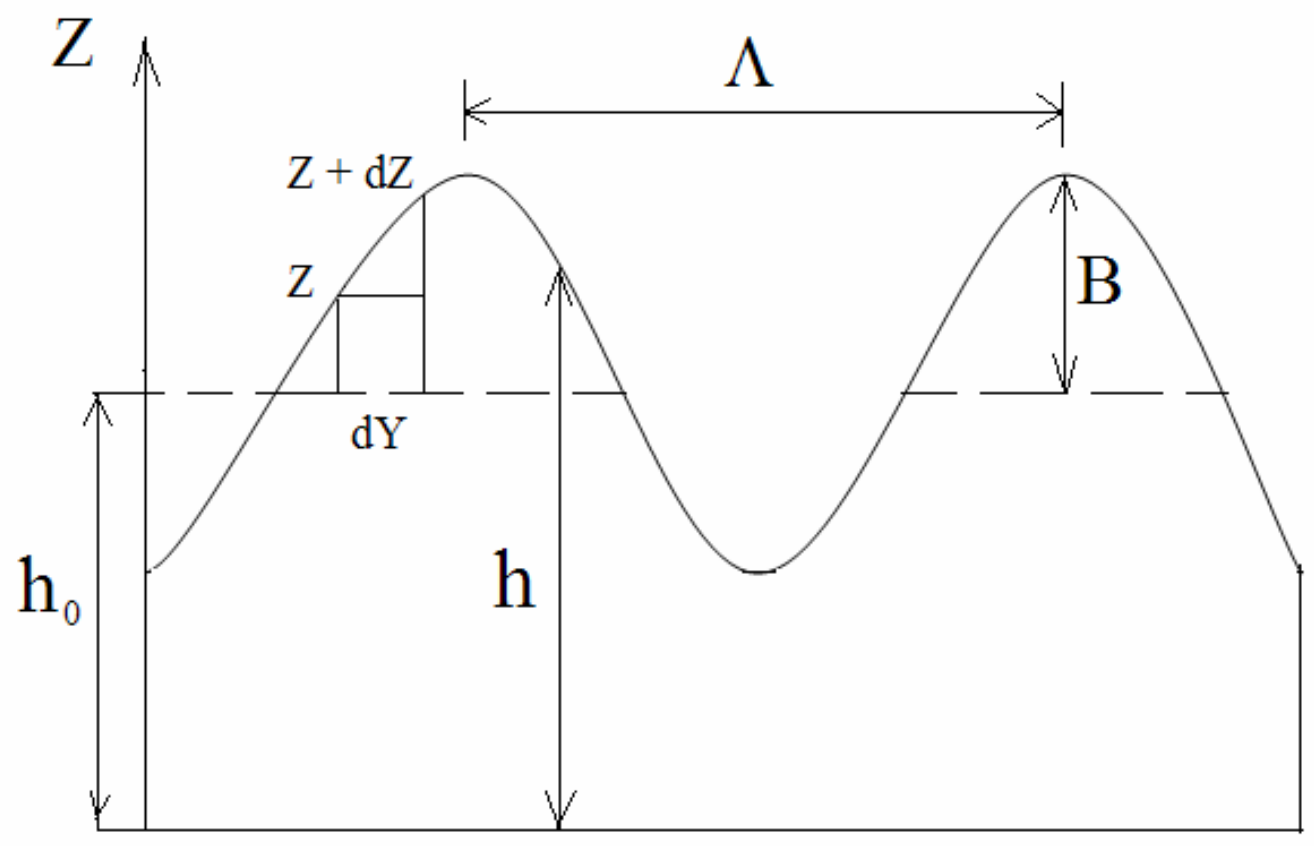

Figure 14. One Fourier components with wavelength $A$ and amplitude $B$ of a corrugated thin film due to surface instabilities. $h_{0}$ is the initial thickness of the film.

composition were replaced by height fluctuations in the surface of annealed films. 
They assumed that these anomalies in the film thickness (corrugations) could be decomposed into Fourier components as is shown in Figure 14.

The excess Gibbs free energy of this corrugated film compared with a completely flat thin film is:

$$
\Delta G=\text { extra area } \times \gamma+\text { effective interfacial energy, }
$$

where the extra area is the difference in surface area between the flat and corrugated films and $\gamma$ is the surface tension (or interface energy). The effective interfacial energy $(\phi(h))$ is defined as the energy required to bring 2 interfaces from the infinity to some separation distance $h$. In the case of interest, $h$ is the thickness of the film and the interfaces are substrate/film and film/vacuum.

By looking at Figure 14, the extra area can be written as

Extra Area $=\int_{y=0}^{y=\Lambda}\left\{\left[(d z)^{2}+(d y)^{2}\right]^{1 / 2}-d y\right\}=\int_{y=0}^{y=\Lambda}\left[\sqrt{1+\left(\frac{d z}{d y}\right)^{2}}-1\right] d y=\frac{1}{2} \int_{y=0}^{y=\Lambda}\left(\frac{d z}{d y}\right)^{2} d y$.

On the other hand, the excess of interfacial energy of one of the Fourier components is

$$
\text { int energy }=\int_{0}^{\mathrm{A}}\left[\frac{d \Phi(h)}{d h} \Delta h+\frac{1}{2} \frac{d^{2} \Phi(h)}{d h^{2}}(\Delta h)^{2}+\ldots\right],
$$

where $\Delta h=h-h_{0}$.

The Fourier components are given by 


$$
\begin{aligned}
& z=z_{0}+B \sin \left(\frac{2 \pi y}{\Lambda}\right) \\
& h=h_{0}+B \sin \left(\frac{2 \pi y}{\Lambda}\right)
\end{aligned}
$$

Plugging equations (45), (46) and (47) into (44) results in

$$
\begin{aligned}
& \Delta G=\frac{1}{2} \gamma \int_{0}^{\Lambda}\left(\frac{d z}{d y}\right)^{2} d y+\int_{0}^{\Lambda} 2 B\left(\frac{d \Phi}{d h}\right) \sin \left(\frac{2 \pi y}{\Lambda}\right) d y+\frac{1}{2} \int_{0}^{\Lambda} B^{2}\left(\frac{d^{2} \Phi}{d h^{2}}\right) \sin ^{2}\left(\frac{2 \pi y}{\Lambda}\right) d y \\
& \text { or, } \\
& \Delta G=\frac{1}{2} \gamma B^{2} \frac{4 \pi^{2}}{\Lambda^{2}} \int_{0}^{\Lambda} \cos ^{2}\left(\frac{2 \pi y}{\Lambda}\right) d y+0+\frac{1}{2} B^{2} \frac{d^{2} \Phi}{d h^{2}} \int_{0}^{\Lambda} \sin ^{2}\left(\frac{2 \pi y}{\Lambda}\right) d y
\end{aligned}
$$

Because the integrals in the Equation (48) are positive, the fluctuations will tend to grow if

$$
\gamma B^{2} \frac{4 \pi^{2}}{\Lambda^{2}}+B^{2} \frac{d^{2} \Phi}{d h^{2}}<0
$$

Hence fluctuations will grow if the characteristic corrugation wavelength is larger than some critical wavelength $\Lambda_{\text {crit }}$ given by

$$
\Lambda>\Lambda_{\text {crit }}=\left(\frac{-4 \pi^{2} \gamma}{\left(d^{2} \Phi / d h^{2}\right)}\right)^{1 / 2}
$$

The film will therefore be unstable and experience spinodal de-wetting whenever $d^{2} \Phi / d h^{2}<0$. This is illustrated in Figure 15, where the effective interfacial potential is plotted as a function of the film thickness for 3 different films: (1) stable, (2) unstable and (3) metastable. For the unstable film, if $h>h^{*}$ the second derivative of the potential will be negative and the film will spontaneously experience spinodal de-wetting. On the other hand, curve (1) shows that the 


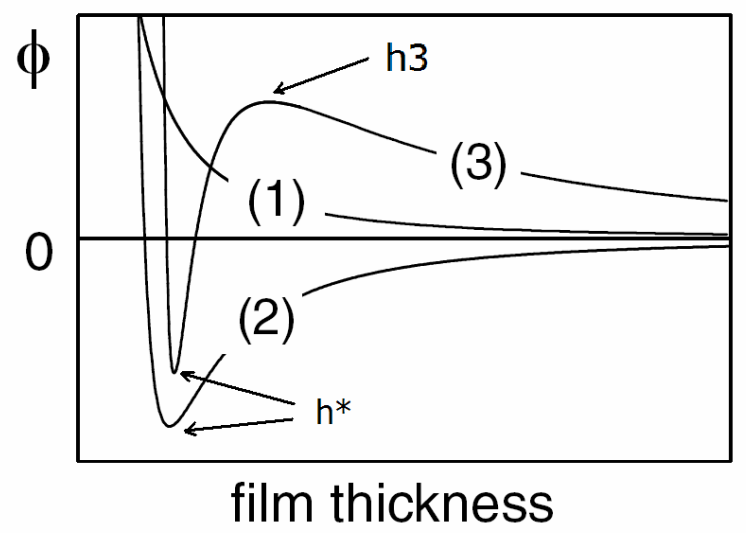

Figure 15. Differences between effective interfacial potential in a film. Curve (1) describes a stable film, (2) an unstable film and (3) a metastable film. After Ref. [38].

global energy minimum can occur when the thickness is infinite and the film is said to be stable. In case (3), the film is unstable for the region $h^{*}<h<h 3$ (where the second derivative of the effective interfacial potential is negative) but for $h>h 3$, the film is stable and it can de-wet only via nucleation. In this case, because the two energy minima are separated by a potential barrier, the film is said to be metastable.

Now suppose that the film is composed of non-polar molecules. A long range interaction may exist, such as a Van der Waals interaction, given by

$$
\Phi(h)=-\frac{A}{12 \pi h^{2}},
$$

where $\mathrm{A}$ is the Hamaker constant. ${ }^{5}$ Using this expression in Equation (50) results in

$$
\Lambda_{c r i t}=h^{2} \sqrt{\frac{8 \pi^{3} \gamma}{A}} .
$$


Hence an experimental proof of spinodal dewetting is to measure the critical wavelength for a given film and determine whether it scales with $h^{2}$, in which case the height instabilities are dominated by a long range Van der Waals interaction.

This one-dimensional model can be extended two dimensions using a height profile of the form ${ }^{5}$

$$
z(\vec{r}, t)=h+\delta h(t) \times \exp \{i \vec{q} \cdot \vec{r}\},
$$

where $r$ and $q=2 \pi \Lambda^{-1}$ are a vector and wavevector, respectively, both parallel to the substrate surface. The amplitude of the corrugations $\delta h(t)$ may be a function of time, and as will be shown later, it can grow exponentially during the early stages of the de-wetting process. 
Because long range Van der Waals forces are expected to be more significant in neutral atoms or non-polar molecules such as polymers, much work has been carried out in order to experimentally confirm spinodal de-wetting in polymer thin films. These de-wetting theories have been extensively applied to polymers

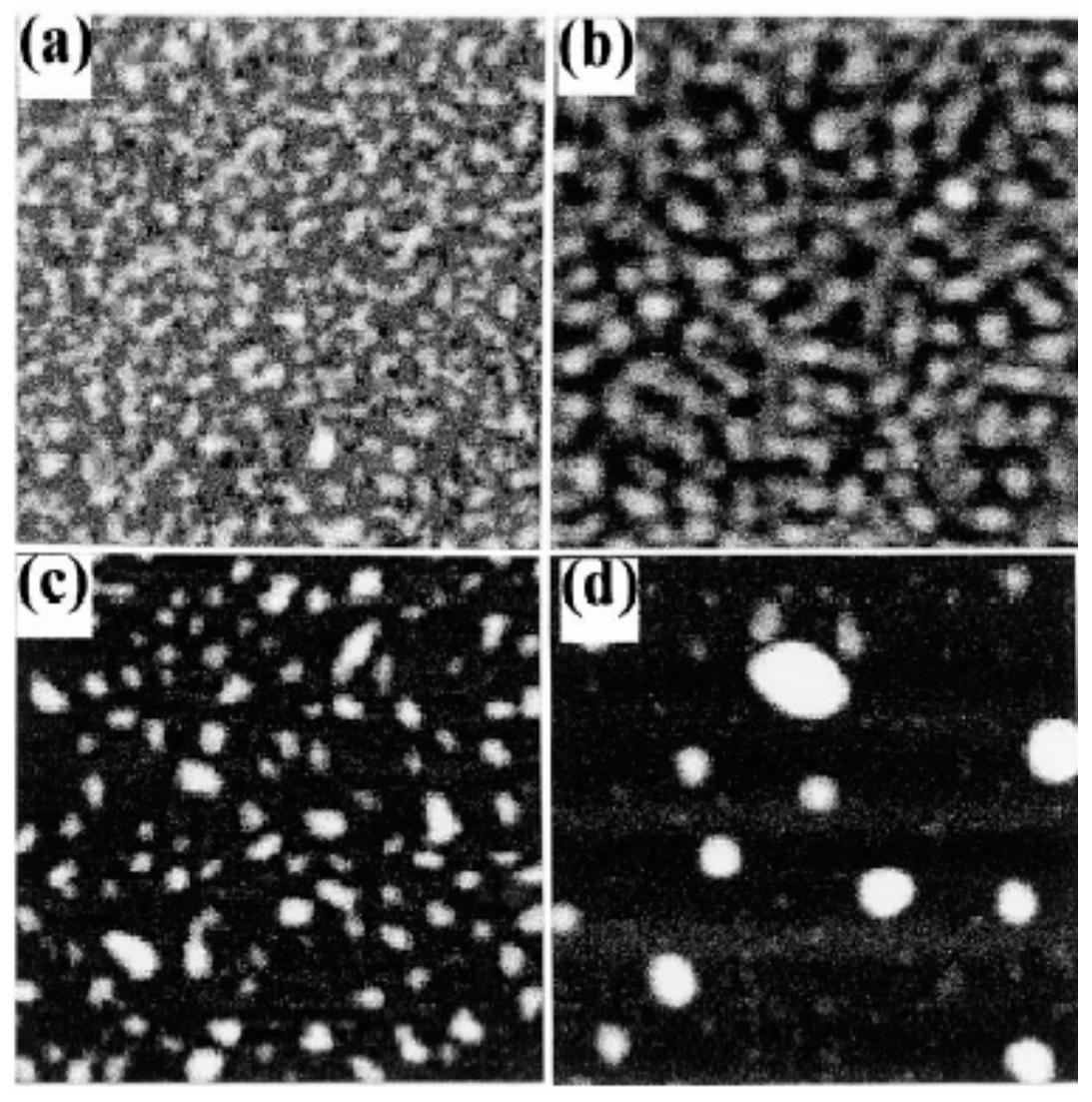

Figure 16. AFM images of a PS film annealed at $115^{\circ} \mathrm{C}$ for: (a) $21 \mathrm{~min}$., (b) $90 \mathrm{~min}$, (c) $7.5 \mathrm{hr}$ and (d) $43 \mathrm{hr}$. Image sizes are $2.5 \times 2.5 \mu \mathrm{m}$. White patterns are hills and black or dark gray represent depressions or holes in the film. From Ref. [5].

because they are amorphous and they behave like liquids above their glass transition, in most cases in the range of $100-150{ }^{\circ} \mathrm{C}$. Also polymers are insulators and they are expected to de-wet due to long range Van der Waals interactions. Figure 16 shows AFM images of a polystyrene (PS) film (45 Á thick) on $\mathrm{Si}(100)$ annealed at $115^{\circ} \mathrm{C}$ for several minutes. ${ }^{5}$ Corrugation patterns are 
observed after 21 minutes of annealing. The patterns increase in size for 90 minutes (figure (b)), the depressions become deeper and larger in area and the hills become taller and wider as well. Later (for $7.5 \mathrm{hr}$ and $43 \mathrm{hr}$ ) the depressions reach the substrate and the last process begins with the breaking up of the film into droplets. Further information can be obtained from the power spectrum of the images (Figure 17). For $21 \mathrm{~min}$. of annealing (white dots), the characteristic wave vector is $25 \mu^{-1}$ as determined from the peak of the power spectrum intensity. At a later time of 90 min., the characteristic wavevector shifts to a slightly lower value. This means that the clusters grew in size during this period of time. The point $q=q_{c}=35 \mu \mathrm{m}^{-1}$ denoted in Figure 17, corresponds to the critical wavevector. Fluctuations with $q<q_{c}$ will tend to grow in time whereas fluctuations with $q>q_{c}$ will decrease with time. The value of $q_{c}$ for this PS film can be determined theoretically using equation (52) and the fact that $q_{c}=2 \pi \Lambda^{-1}$. For PS, $\gamma=0.0319 \mathrm{~J} / \mathrm{m}^{2}, h=4.5 \mathrm{~nm}$ and $A=7.42 \times 10^{-20} \mathrm{~J} .^{5}$ For these values $\Lambda=$ $0.17 \mu \mathrm{m}$ and $q_{c}=38 \mu \mathrm{m}^{-1}$, the latter of which agrees remarkably well with the experimental value. In the case of transition metals like cobalt, if long range Van der Waals dominated, theoretically a value of $\Lambda_{c}=1 \mu \mathrm{m}$ would be expected using $\gamma=2 \mathrm{~J} / \mathrm{m}^{2}, h=4.0 \mathrm{~nm}$ and $A=20 \times 10^{-20} \mathrm{~J}$. This critical wavelength requires taking AFM images of at least $2 \times 2 \mu \mathrm{m}^{2}$ in order to detect $\Lambda_{c}$ in order to avoid artifacts from the edge of the images.

It was mentioned above that a good method to verify if a film undergoes spinodal de-wetting is to measure the characteristic wavelength as a function of thickness. In Figure 18(a) another experiment is depicted ${ }^{38}$ that measured the wavelength of a PS film as a function of the film thickness. In one case the film was deposited on silicon with a SiO overlayer $191 \mathrm{~nm}$ thick (filled circles). It is evident in the plot that the wavelength is a function of $h^{2}$, indicating a spinodal mechanism. On the other hand, from these data and using Equation (50), it is possible to infer the sign of $d^{2} \Phi / d h^{2}$ as a function of film thickness. For the PS film deposited on the $191 \mathrm{~nm}$ SiO layer, $d^{2} \Phi / d h^{2}$ is negative (see Figure 18(b)), and therefore the film is unstable and should undergo spinodal de-wetting. Also 


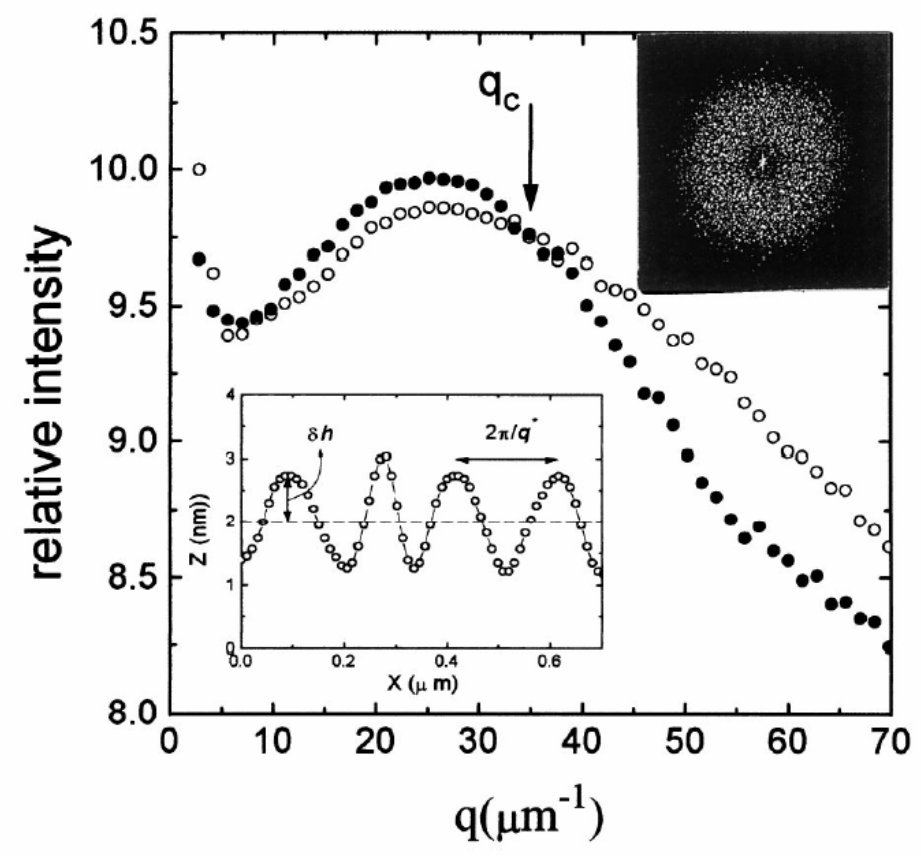

Figure 17. Radial average of the power spectrum as a function of the wave vector $\boldsymbol{q}\left(q^{2}=q_{x}^{2}+q_{y}^{2}\right)$ for a PS film $45 \AA$ Á thick. White and black dots represent the spectrum for $21 \mathrm{~min}$. and $45 \mathrm{~min}$. of annealing at 115 ${ }^{\circ} \mathrm{C}$, respectively. The top right inset shows the 2D power spectrum obtained from FFT. The bottom left inset shows the profile of the corrugations in the film. After Ref. [5].

it can be inferred from Figure 18(b) that films deposited on the thinner oxide layer can undergo either spinodal $\left(\Phi^{\prime \prime}<0\right)$ or heterogeneous nucleation $\left(\Phi^{\prime \prime}>0\right)$ depending on the initial thickness of the oxide layer. 

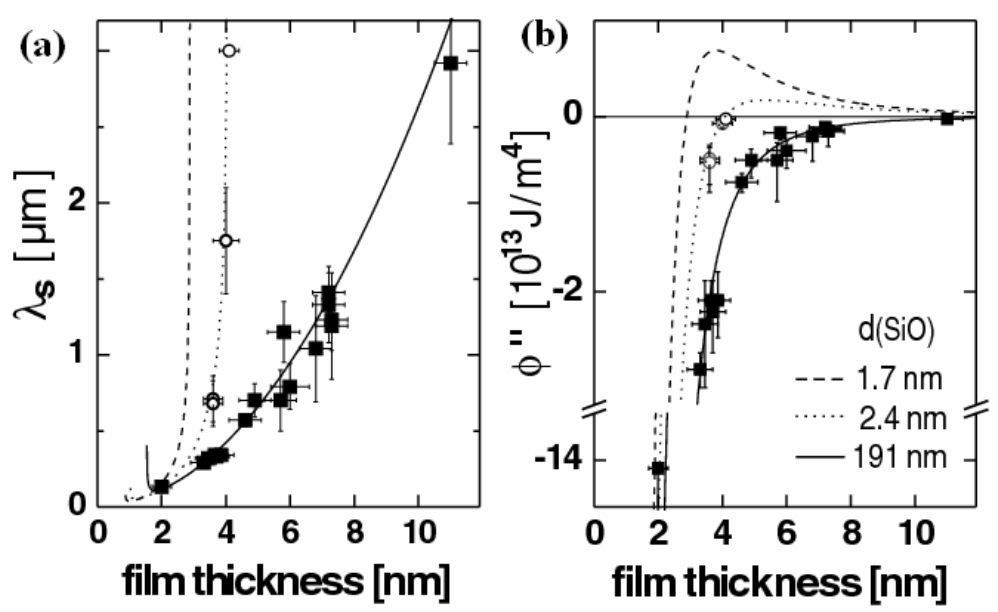

Figure 18. (a) Characteristic wavelength of a PS film as a function of thickness. The PS film was deposited on top of $2.4 \mathrm{~nm}$ of SiO (open circles) and $191 \mathrm{~nm}$ of SiO (filled squares). (b) Second derivative of the effective interfacial potential as a function of the film thickness. After Ref. [38].

Up to now the dependence of the corrugations on the initial film thickness, film surface energy and effective potential has been considered. Now, consider the time dependence in one dimension. The growth of these fluctuations is related the the mass flow of material on the surface. Mass flow can be expressed as the movement of a fluid driven by pressure gradients:

$$
\Delta P=\Delta P_{\text {cap }}+\Delta P_{\text {interaction }}=-\gamma \frac{d^{2} z}{d y^{2}}+\frac{d^{2} \Phi}{d h^{2}} \Delta h,
$$

where the $\Delta P_{\text {cap }}$ is the gradient due to capillarity and $\Delta P_{\text {interaction }}$ is the gradient due to the effective interaction potential. On the other hand, mass flow can be estimated as the flow between 2 fixed walls driven by a pressure gradient as

$$
\text { flow_rate }=Q=\frac{h_{0}^{3}}{12 \eta} \frac{d(\Delta P)}{d y},
$$

where $\eta$ is the viscosity of the fluid. Conservation of volume requires that 


$$
\frac{\partial h}{\partial t}=-\nabla Q=-\frac{d Q}{d y}=\frac{h_{0}^{3}}{12 \eta} \frac{d^{2}(\Delta P)}{d y^{2}}
$$

Hence from equations (54), (55) and (56) and solving the differential equation for $h$, one obtains

$$
\left(h-h_{0}\right)=\left(h-h_{0}\right)_{t=0} \exp (t / \tau)
$$

or,

$$
\delta h=\delta h_{0} \exp (t / \tau)
$$

where $t$ is the time, $h$ is the actual height, $h_{0}$ the film height at $t=0$, and $\tau$ a characteristic time that has the form

$$
\tau=-\frac{3 \eta \Lambda^{2}}{\pi^{2} h_{0}^{3}}\left(\frac{2 \pi^{2} \gamma}{\Lambda^{2}}+\left(\frac{d^{2} \Phi}{d h^{2}}\right)_{h=h_{0}}\right)^{-1}
$$

This expression is valid for any long range interaction. In the case of Van der Waals forces the critical characteristic time can be deduced to have the form

$$
\tau_{c}=48 \pi^{2} \eta \gamma A^{-2} h_{0}^{5}
$$

The exponential dependence of the average height of the corrugations on time can be observed in Figure 19 for a PS film (45 $\AA$ thick) annealed at $115^{\circ} \mathrm{C} .{ }^{5}$ From $t=0$ to $1.5 \mathrm{hr}$, the film amplitude grows exponentially as predicted under the model of pressure gradients of mass flow. At $1.5 \mathrm{hr}$ the amplitudes of corrugations is about $1.2 \mathrm{~nm}$, hence the peak to peak amplitude of the corrugations is approximately $2.4 \mathrm{~nm}$ less than the film thickness of $4.5 \mathrm{~nm}$. However, deviations from the exponential grow occur after $1.5 \mathrm{hr}$, even after dewetting takes place. By fitting the exponential curve, an experimental characteristic time of $198 \mathrm{~min}$ is obtained. 


\subsubsection{Kinetic Theory of De-wetting on Solids}

Consider adatoms on the surface of a film of thickness $h_{0}$. Atoms might move on the surface, they might remain still or they might be incorporated into the bulk. Therefore there will be a certain number of active atoms producing small thickness fluctuations $\delta h(\vec{x}, t)$ at a time $t$ and position $\vec{x}$ such that the local thickness is

$$
h=h_{0}+\delta h(\vec{x}, t),
$$

where $\delta h<<h_{0}$. The thickness fluctuations are given by: $:^{39,40}$

$$
\frac{\partial h}{\partial t}=-\vec{\nabla} \cdot \vec{J},
$$

where $\vec{J}$ is the surface current $\left(\mathrm{m}^{2} / \mathrm{s}\right)$, which in turn has two contributions such that

$$
\vec{J}=\vec{J}_{\gamma}+\vec{J}_{\Phi}
$$

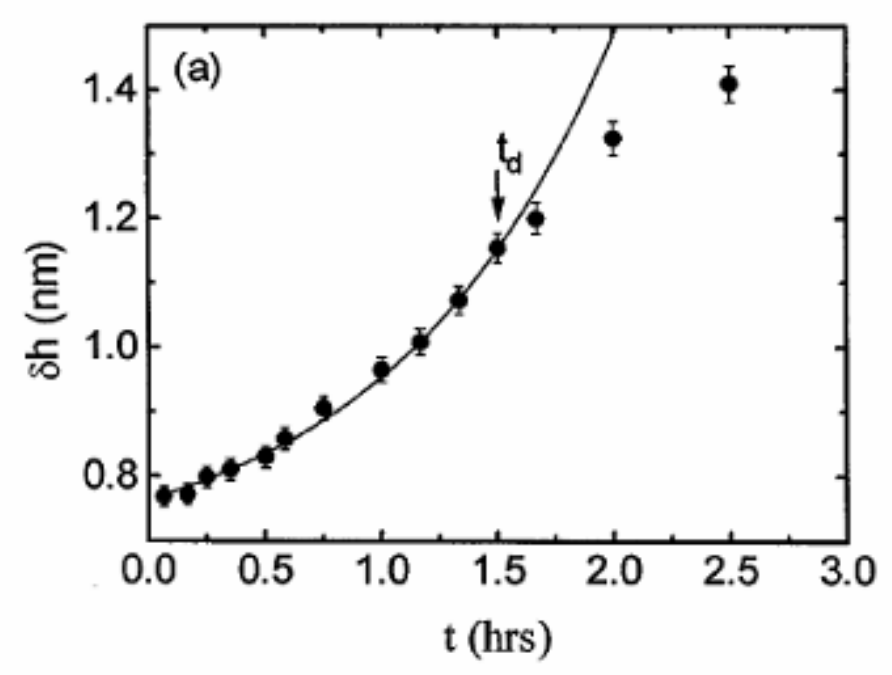

Figure 19. Average amplitude of the corrugations for a PS film (45 Á thick) annealed at $115^{\circ} \mathrm{C}$. After Ref. [5]. 


$$
\begin{aligned}
& \vec{J}_{\gamma}=\Gamma \vec{\nabla}\left(\gamma \nabla^{2} h\right) \\
& \vec{J}_{\Phi}=-\Gamma \vec{\nabla}\left(\Phi^{\prime}(h)\right)
\end{aligned},
$$

where $\Gamma$ is the atomic mobility and $\gamma$ the surface tension. The first term $\left(\vec{J}_{\gamma}\right)$ is Mullins' diffusion expression ${ }^{39}$ that predicts the movement of atoms on a surface due to differences in curvature of the film. In other words, atoms placed in regions with small curvatures (flat surfaces) will have a tendency to diffuse to places with high curvatures (rougher places in the film) leading to the smoothing of the surface. Hence Mullins' term is analogous to capillarity in fluids because it favors the smoothing of the films as well. The second term $\left(\vec{J}_{\Phi}\right)$ is the surface current due to Casimir interactions across the film and is expressed as a thermodynamic potential $\Phi(h)$. In general these long-range interactions, such as Van der Waals forces, are attractive and therefore oppose wetting forces.

The conditions for film stability and the characteristic corrugation wavelength can be obtained from Equation (62) by expanding the interfacial potential about the film's initial thickness $h_{0}$,

$$
\begin{aligned}
& \Phi^{\prime}(h)=\Phi^{\prime}\left(h_{0}+\delta h\right) \\
& \Phi^{\prime}(h)=\Phi^{\prime}\left(h_{0}\right)+\delta h \Phi^{\prime \prime}\left(h_{0}\right)
\end{aligned}
$$

so that

$$
\begin{aligned}
& \nabla^{2} h=\nabla^{2} \delta h \\
& \frac{\partial h}{\partial t}=\frac{\partial(\delta h)}{\partial t}
\end{aligned}
$$

Plugging (64) and (65) into (62) results in

$$
\frac{\partial(\delta h)}{\partial t}=\Gamma \nabla^{2}\left[\Phi^{\prime}\left(h_{0}\right)+\delta\left(h(\vec{x}, t) \Phi^{\prime \prime}\left(h_{0}\right)-\gamma \nabla^{2} \delta h\right]\right.
$$


and

$$
\Phi^{\prime}\left(h_{0}\right)=0 .
$$

The first derivative evaluated at $h_{0}$ is zero because $h_{0}$ is the equilibrium position. Therefore,

$$
\frac{\partial(\delta h)}{\partial t}=\Gamma\left[\Phi^{\prime \prime}\left(h_{0}\right) \nabla^{2} \delta h-\gamma\left(\nabla^{2}\right)^{2} \delta h\right] .
$$

This differential equation can be solved assuming the solutions

$$
\begin{aligned}
& \delta h=A_{q}(t) \exp (i \vec{q} \cdot \vec{x}) \\
& A_{q}(t)=A_{q}(0) \exp \{\omega(q) t\}
\end{aligned}
$$

where $\vec{q}$ is some wavevector on a plane parallel to the substrate's surface, $\vec{x}$ is a vector position on the film's surface, $A_{q}$ is the time-dependent amplitude of the film's corrugations, and $\omega$ some characteristic frequency. Hence it is evident that

$$
\begin{aligned}
& \nabla^{2} \delta h=-q^{2} \delta h \\
& \left(\nabla^{2}\right)^{2} \delta h=q^{4} \delta h
\end{aligned}
$$

and thus Equation (68) becomes

$$
\frac{\partial A_{q}(t)}{\partial t}=\omega(q) A_{q}(t)
$$

with

$$
\omega(q)=\Gamma\left[-\Phi^{\prime \prime}\left(h_{0}\right) q^{2}-\gamma q^{4}\right] .
$$

These two equations are the central results of the kinetic theory of a solid's dewetting process. If $\omega(q)$ is negative, the corrugations on the film will smooth out, but if $\omega(q)$ is positive, the corrugations will grow exponentially with time. One condition for this exponential growth to occur is that: 


$$
\Phi "\left(h_{0}\right)<0 \text {. }
$$

The surface instabilities are depicted in Figure 20 for $\Phi^{\prime \prime}<0$, where $q_{c}$ is a critical wave vector. For $q>q_{c}$ the frequencies are negative and the corrugations exponentially decay with time (smoothing out of the surface). On the other hand, for $q<q_{c}$ the frequencies are positive and the corrugations grow exponentially with time, leading to the de-wetting of the film. In Figure $21 q_{\text {early }}$ is the wave vector leading to the fastest growth of the height instabilities and can be calculated as

$$
\left.\frac{\partial \omega}{\partial q}\right|_{q}=0
$$

Hence

$$
-\Phi "\left(h_{0}\right)-2 \gamma q_{\text {early }}^{2}=0
$$

Solving for qearly results in

$$
\begin{aligned}
& q_{\text {early }}=\sqrt{\frac{-\Phi "\left(h_{0}\right)}{2 \gamma}} \\
& \lambda_{\text {early }}=\frac{2 \pi}{q_{\text {early }}}=\sqrt{\frac{8 \pi^{2} \gamma}{-\Phi^{\prime \prime}\left(h_{0}\right)}} .
\end{aligned}
$$

These equations are completely general because they do not make any assumptions regarding the shape of the interaction potential. On the other hand, it is possible to calculate $q_{c}$ by just setting the equation (72) equal zero, i.e.,

$$
\left[-\Phi "\left(h_{0}\right) q^{2}-\gamma q^{4}\right]=0
$$

so that 


$$
q_{c}=\sqrt{\frac{-\Phi "}{\gamma}},
$$

and therefore

$$
q_{c}=q_{\text {early }} \sqrt{2} .
$$

The critical wavelength is then equal to:

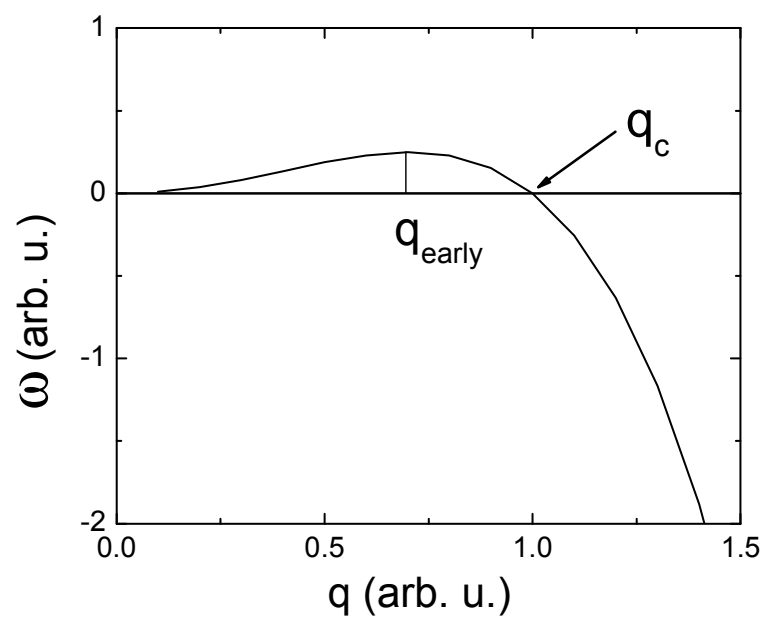

Figure 20. Variation of the frequency with the characteristic wave length of the corrugations in a thin film.

$$
\Lambda_{\text {crit }}=\left(\frac{-4 \pi^{2} \gamma}{\Phi "(h)}\right)^{1 / 2}
$$

A surprising result is that the form of $\Lambda_{\text {crit }}$ is exactly the same as the $\Lambda_{c}$ calculated for fluids in the previous section (Equation (50)).

The value of the early wavelength is determined by the type of long range interaction present in the film. For a Casimir interaction such as the Van der 
Waals force, the early wavelength has a form analogous to the one obtained for liquids, namely

$$
\lambda_{\text {early }}=h^{2} \sqrt{\frac{16 \pi^{3} \gamma}{A}} .
$$

\begin{tabular}{c|c}
\hline $\begin{array}{c}\text { Film } \\
\text { Thickness (nm) }\end{array}$ & $\lambda_{\text {early }}$ \\
\hline 4 & $\sim 1 \mu \mathrm{m}$ \\
10 & $\sim 7 \mu \mathrm{m}$ \\
100 & $\sim 1 \mathrm{~mm}$ \\
\hline
\end{tabular}

Table 2. Variation of the early wavelength with the film thickness for a long range Van der Waals interaction. The thickness calculated for a cobalt film with $\gamma=2 \mathrm{~J} / \mathrm{m}^{2}$ and $A=20 \times 10^{-20} \mathrm{~J}$ (typical for metals).

In order to illustrate the strong dependence of the early wavelength with the film thickness $h$, Table 2 shows the computed values of the early wavelength for a cobalt thin film of different thicknesses using Equation (81). Clearly $\lambda_{\text {early }}$ increases from $1 \mu \mathrm{m}$ for a film thickness of $4 \mathrm{~nm}$ to 1 millimeter for a thickness of $100 \mathrm{~nm}$. It is evident that height instabilities can be observed only in thin films where $h<10 \mathrm{~nm}$.

Thus far it has been shown that if the second derivative of the interfacial potential is negative the film is unstable and that it ruptures via spinodal de-wetting. However there are cases where the second derivative of the interfacial potential can be either positive or negative depending on the thickeness of the film (Figure 15); when this happens, the film is said to be metastable. As explained previously, a metastable film has a stable and unstable regions separated by a potential barrier. Inside the unstable region (where $\Phi^{\prime \prime}<0$ ) the film might break via spinodal de-wetting, however, in the region where $\Phi^{\prime \prime}>0$ the film might dewet via nucleation. This normally happens when impurities or defects are 
present in the film or at the substrate/film interface. This dewetting mechanism is called heterogeneous de-wetting. If impurities are not present, then the film still might experience nucleation by thermally overcoming the potential barrier. In this case the de-wetting process is called homogeneous de-wetting. In a real system, the three de-wetting mechanisms may coexist, thus making it very difficult to determine experimentally which process is dominant.

\subsubsection{Casimir Interactions}

In 1948 the Dutch physicist H. B. Casimir ${ }^{41}$ using Quantum Electrodynamics, predicted that two uncharged metallic plates placed in a perfect vacuum will attract each other. This attraction is due to the modification of the zero point energy between the plates compared with the empty space producing a net force (in S.I. system) per unit of area equal to

$$
F(d)=-\frac{\pi \hbar c}{240 d^{4}} \times 10^{-1}
$$

where $\hbar$ is Planck's constant divided by $2 \pi, c$ is the speed of light and $d$ the separation between the 2 plates. Recently this phenomenon was confirmed experimentally with high accuracy. ${ }^{42}$ The origin of this force results from the fact that even in a perfect vacuum at zero absolute temperature there is a fluctuating electromagnetic field having a mean energy equal to half the energy of a photon. These fluctuations of the electromagnetic field in vacuum are modified if, for example, two perfect mirrors are placed close each other. Inside of the cavity, the electromagnetic field can be amplified if the distance between the plates is equal to multiple numbers of half wavelengths of the radiation. ${ }^{43}$ In this case the electromagnetic pressure inside the cavity is larger than outside of it and the mirrors experience a repulsive force. For other wavelengths, the pressure field will be stronger outside the cavity and the plates will attract each other. Wavelengths longer than the separation between the plates cannot reside in the cavity, therefore there will be less number of photons able to produce a pressure 
inside the plates. This generates an unbalance between attractive and repulsive pressures in favor of the attraction of the plates. The strong character of this interaction can be observed in the following example. If two flat plates with an area of $1 \mathrm{~cm}^{2}$ are separated by $1 \mu \mathrm{m}$, the Casimir force is of the order of $10^{-7} \mathrm{~N}$; however if the separation is of the order of $1 \mathrm{~nm}$, then the pressure exerted on the flat plates is equivalent to 1 atmosphere.

On the other hand, a classical explanation for this effect is given by Lifshitz ${ }^{44}$ proposing that in any material fluctuations of the atoms' electronic charge density exist even at zero absolute temperature. These fluctuations of the electromagnetic field generate transient dipoles moments in the atoms which in turn affect other atoms in the material. These fluctuations give rise to dissipation forces called London forces. The magnitude of these forces is weak compared to covalent or ionic interactions between molecules, and therefore they are more important between neutral atoms like noble gases or non-polar molecules such as those that compose many polymers. London dissipative forces (fluctuating dipoles inducing other dipoles in the material) are not the only forces acting on neutral atoms. Dipoles induced by permanent dipoles and permanent dipoledipole interactions are part of what are called Van der Waals interactions. Therefore the London force constitutes a branch of Van der Waals interactions and for this reason it is sometimes called a London-Van der Waals force or bosonic Casimir force because the responsible of transmitting the force is the electromagnetic field (photons). The London energy (in S.I. system) between 2 neutral atoms is described by

$$
\Phi(d)=-\frac{3 \alpha^{2} h v}{4\left(4 \pi \varepsilon_{0}\right)^{2} d^{6}},
$$

where $\alpha$ is the polarizability, $h$ is Planck's constant, $v$ is the frequency of fluctuation, $\varepsilon_{0}$ the dielectric permittivity of vacuum, and $d$ the separation distance between the atoms. 
Hamaker extended the London-Van der Waals interaction between two neutral atoms to macroscopic flat surfaces through the potential

$$
\Phi(h)=-\frac{A_{H}}{12 \pi h^{2}},
$$

where $A_{H}$ is the Hamaker constant and $h$ the thickness of the material or thin film. The Hamaker constant is positive for interactions between identical molecules but it can be zero or even negative when they interact through a third medium; in which case the Van der Waals interaction is repulsive. Table 1 shows the value of the Hamaker constant for different materials.

\begin{tabular}{c|c}
\hline Material & $\begin{array}{c}\text { Hamaker Constant } A_{H} \\
\left(10^{-20} \mathrm{~J}\right)\end{array}$ \\
\hline Water & 3.7 \\
Fused Quartz & 6.3 \\
Metals & $20-50$ \\
Polymers & $4-8$ \\
\hline
\end{tabular}

Table 3. Hamaker constant for different materials. After Ref [45].

It was mentioned before that bosonic Casimir interactions are forces that are transmitted through photons. Recently, however, another interaction mediated by non-relativistic electrons, ${ }^{46,47}$ the so called fermionic Casimir interaction, was predicted to occur. Here, the bosonic field is replaced by a fermionic field with a wavelength related to the Fermi wave vector $k_{F}$. In analogy to the bosonic Casimir interaction, two cavities immersed in the fermionic sea will interact when the fermion waves interact, provided that the wavelengths are comparable with the size and separation of the cavities. Experimentally, this interaction is expected to be more important in metals, where the itinerant electrons create a fermionic gas. For instance, it has been proposed that the finite thickness in a thin film of a metal might lead to a repulsive fermionic interaction of the form ${ }^{48}$

$$
\Phi(h) \cong-\frac{\varepsilon_{F}}{a h},
$$


where $\varepsilon_{F}$ is the Fermi energy of the metal, $a$ is the lattice parameter and $h$ the thickness. If this interaction dominates the behavior of metallic thin films, it may play an important role in the de-wetting process.

\subsection{INTERMEDIATE AND LATE STAGES OF DE-WETTING}

As stated before, the focus of this thesis is to understand the formation of cobalt clusters on sapphire rather than to study their late evolution. However a few ideas will be given about the intermediate and late stages of de-wetting in order to present a complete picture of the de-wetting process.

In the early stages of de-wetting, height instabilities grow exponentially with time. When these instabilities $\mathrm{h}(\mathrm{t})$ are comparable with the initial film thickness $H$, i.e. $h(t) \sim H$, an intermediate stage occurs which is characterized by the nucleation of holes whose size and shape evolve with time. In Figure 21 it is possible to observe the intermediate stage of an annealed PS film. At $t=770 \mathrm{~s}$ the height instabilities reach the $\mathrm{Si}$ substrate and 3 holes nucleate on the film (top left side). At $t=2270 \mathrm{~s}$ and $t=4220 \mathrm{~s}$ the number of holes and their diameters increase.

$770 \mathrm{~s}$

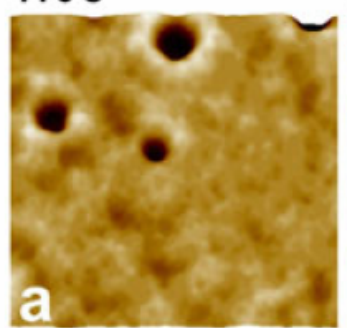

$1 \mu \mathrm{m}$
$2270 \mathrm{~s}$

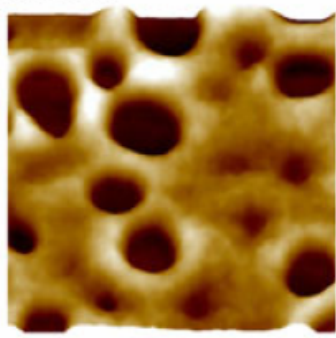

$4220 \mathrm{~s}$

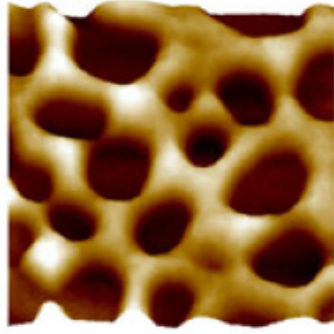

$6500 \mathrm{~s}$

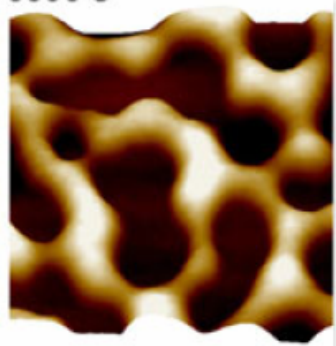

Figure 21. PS film ( $3.9 \mathrm{~nm}$ thick) on top of SiO annealed at $53^{\circ} \mathrm{C}$. The black spots in the images represent holes in the PS film. After Ref. [49].

Finally, at $t=6500 \mathrm{~s}$ the holes are so close to each other that they coalesce with neighbors forming larger vacancies. In general there are 2 main characteristics of the holes: their diameter and their outer rim. Figure 22 shows an AFM image 
of a nucleated hole. The bright region around the hole is the characteristic rim with the accumulated material coming from inside of the nucleated hole. In general, for liquids and polymers the holes grow in two modes. For Newtonian liquids, where a non slippery condition exists between the liquid and the

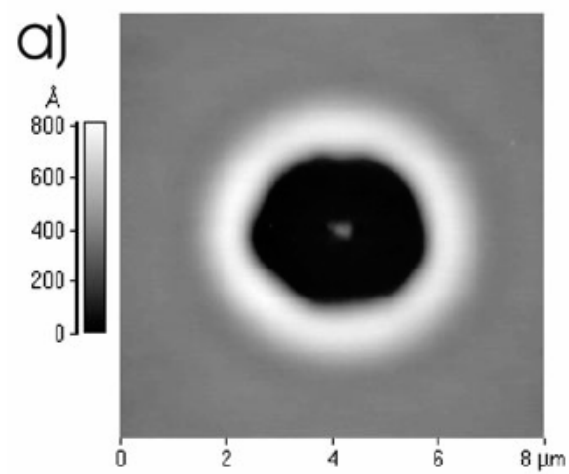

Figure 22. AFM image of a PS film $41 \mathrm{~nm}$ thick. The black spot is a nucleated hole in the film surrounded by a rim of accumulated material in the outside edges. After Ref. [51].

substrate (i.e. the relative velocity of the liquid and solid at the interface is zero), the holes grow with constant velocity, or in other words, as $R \sim t$, where $R$ is the radius of the hole and $t$ the time. On the other hand, for perfect slippery conditions a power law $\mathrm{R} \sim \mathrm{t}^{2 / 3}$ has been measured experimentally. ${ }^{50}$

After the holes have fully developed, they start to coalesce with each other, creating larger holes in the film. This process continues until isolated islands are left in the substrate (see Figure 23 and Figure 24) when the de-wetting process is complete. The late stages of de-wetting consist of the evolution of de-wetted islands (either liquid drops or a solid's clusters) as a function of time. The final shape of the islands are influenced by gravity, diffusion, and Ostwald ripening among other factors. 


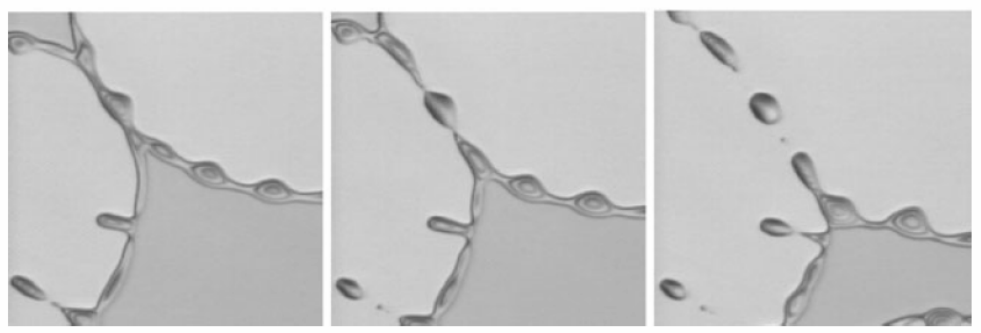

Figure 23. Late stage of de-wetting of a PS film on silicon. Lighter zones are holes in the PS film and darker regions are places where the PS film still wets the substrate. After Ref. [52].

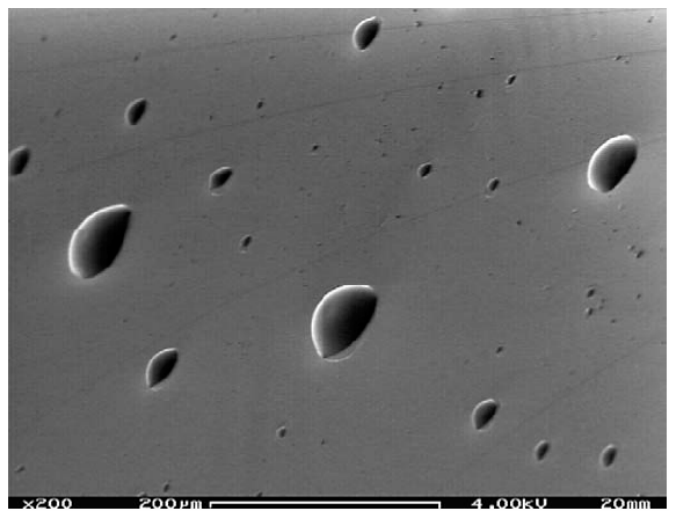

Figure 24. SEM image showing the de-wetting of a $500 \mathrm{~nm}$ aluminum thin film on sapphire (001) annealed at $900{ }^{\circ} \mathrm{C}$ during 120 minutes. After Ref. [53]. 


\section{EXPERIMENTAL PROCEDURES}

The complete experiment is carried out in ultra high vacuum (UHV) conditions in order to avoid the contamination and oxidation of the samples. Figure 25 depicts the vacuum chambers involved in the experiment. The substrate is installed in the loading lock (LL) and then moved in vacuum using the transfer chamber (UFO) to the metal beam epitaxy chamber (MBE) where the thin film is grown. After that, the sample is transported to the scanning probe microscope chamber (SPM) where the actual experiment takes place.

The experimental procedure can be divided into the following steps:

1.- Substrate's preparation and cleaning

2.- Cobalt thin film growth

3.- Imaging of the thin film during annealing

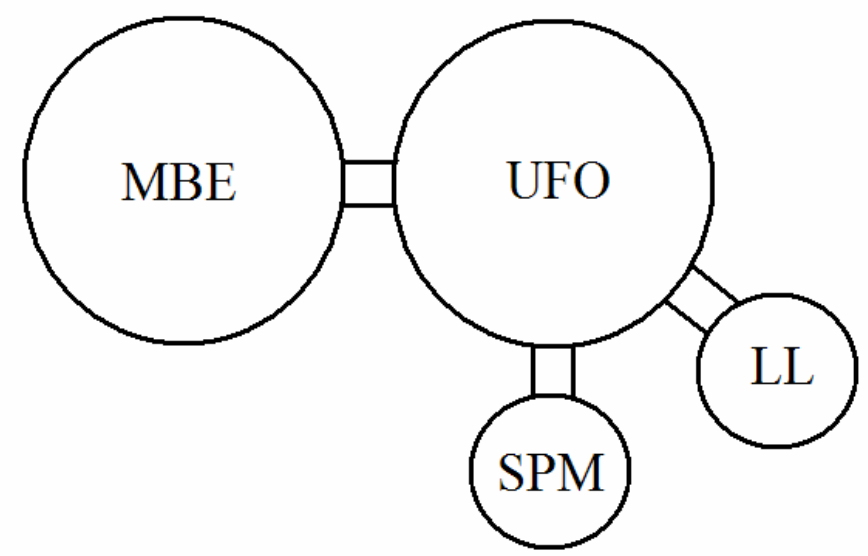

Figure 25. Configuration of UHV chambers. The samples are introduced through the Loading Lock (LL) in the UHV system and transferred to the other chambers inside the UFO. The film is growth in the MBE chamber and imaged in the SPM . 


\subsection{SUBSTRATE PREPARATION AND CLEANING}

Sapphire wafers 2" in diameter and 330 micron thick were purchased from Saint Gobain Crystals. The wafers were cut into $10 \times 2 \mathrm{~mm}^{2}$ rectangles using a diamond cutter and flushed with $\mathrm{N}_{2}$ gas to remove particles in the surface. After that the substrates were installed inside an alumina crucible (Vesuvius) $99.8 \%$ pure and then placed inside a Lindberg/Blue Tube Furnace rated with maximum working temperature of $1700^{\circ} \mathrm{C}$ and a temperature uncertainty of $\pm 1^{\circ} \mathrm{C}$. For this purpose we used an alumina tube (Vesuvius) $99.8 \%$ pure. The substrates were

cleaned by annealing only in air ${ }^{54}$ at $1400{ }^{\circ} \mathrm{C}$ during 1 hour. The temperature ramp to heat up and to cool down the sample was linear with a temperature rate of $272{ }^{\circ} \mathrm{C}$ per hour. This is the fasted rate allowed by the tube manufacturer to avoid cracks and deformations.

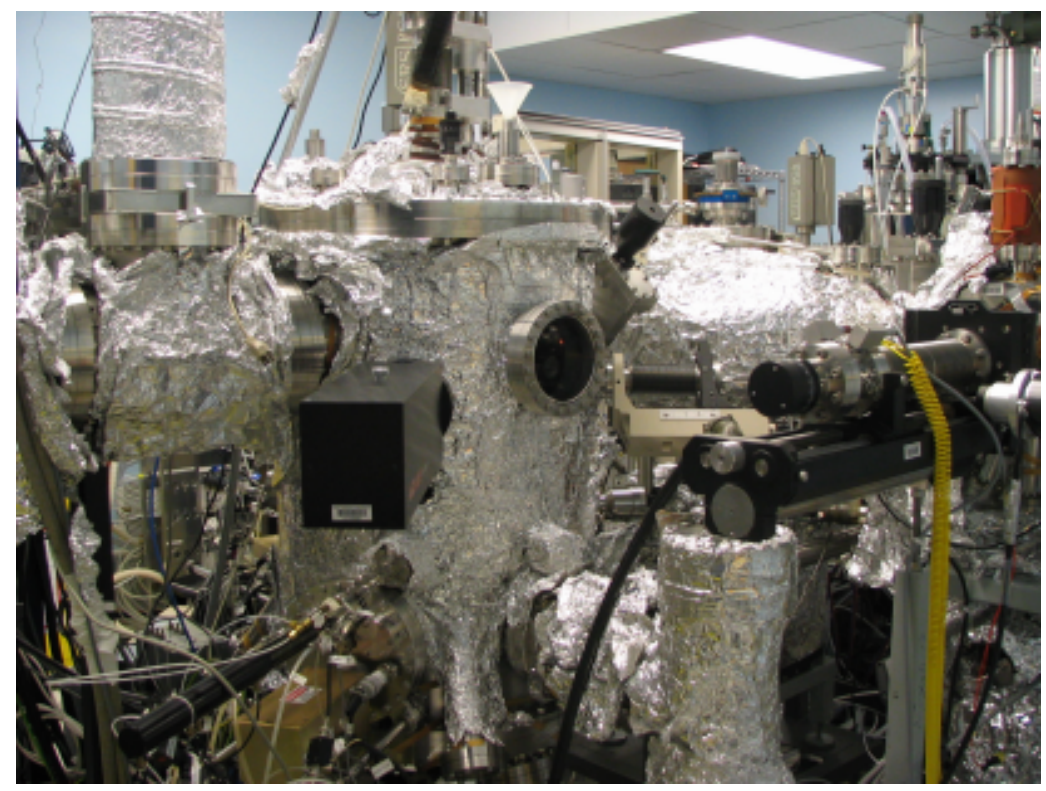

Figure 26. Actual Picture of the MBE chamber. 
The annealing only procedure helps to evaporate contaminants from the sapphire surface and produces terraces with heights of single and multiple atomic mono-steps. This cleaning also avoids the use of chemical etching that normally leaves residues on the substrates. The visualization of terraces and atomic steps is normally a good indication of a clean surface. After the annealing, the substrate was mounted in an Omicron variable temperature sample holder that uses a Pyrolytic Boron Nitride (PBN) element to indirectly heat up the sample. After that, the cleaned substrate was loaded into an UHV chamber through a loading lock (see Figure 25) and then moved to an Omicron variable temperature SPM (also in UHV). The substrates were imaged using non-contact AFM at room temperature to observe if the surface was clean prior cobalt growth.

\subsection{COBALT THIN FILM GROWTH}

The growth of the cobalt film was carried out in the MBE chamber shown in Figure 26. The MBE source used for the thin film growth (MDC e-vap 4000) is a multi-pocket system allowing the installation of 4 different materials in the same gun. The MBE works as follows:

- The material to be evaporated (target) is installed inside a crucible of $6 \mathrm{cc}$.

- A tungsten filament (placed at about $3 \mathrm{~cm}$ from the crucible) is heated up and electrons are emitted.

- The crucible is normally grounded and the filament is held at a high voltage of $-5 \mathrm{KV}$ In this way the emitted electrons are accelerated towards the target; the collisions produce the melting and evaporation of the material in the crucible.

- Coils located near the filament and the crucible control the trajectory of the electrons in order to center them right in the middle of the crucible. The coils also allow sweeping the e-beam around the material using different patterns (circles, spirals, etc) in order to heat the surface of the target more uniformly. The position of the beam must be monitored through a 
dedicated viewport in order to ascertain that the electron beam is hitting the target; furthermore, it must be remembered that the position of the beam may change as the target heats up, especially for a magnetic material such as Co which becomes non-magnetic at high temperatures.

The main advantages of MBE growth are that it makes it possible to evaporate high melting point $\left(>1000{ }^{\circ} \mathrm{C}\right)$ materials and that by controlling the power of the e-beam filament it allows to melt only the surface of the target, reducing the formation of alloys between the target and crucible. A disadvantage of the MBE is that the evaporation of low melting point materials (i.e. aluminum) is difficult due to the fact that small changes in the filament current or the e-beam centering in the target produce a large increment in the evaporation rate, with the risk of coating unexpectedly crystal monitors, viewports and producing rough films. Another disadvantage is that the e-beam produces a charging effect in crucibles fabricated from insulator materials which decreases the electron beam energy hitting the target. For our cobalt target we used an MDC alumina crucible $(6 \mathrm{cc})$, a filament current of $\sim 0.1 \mathrm{~A}$, a power of $7-12 \%$, a high voltage of $-5 \mathrm{KV}$ and a circular sweeping pattern.

Two quartz crystal monitors allowed us to estimate the thickness of the cobalt thin film with the aid of a Maxtek MDC-360 quartz crystal deposition controller. The quartz crystal was purchased from Sigma Instruments and is a flat circular plate of $1.40 \mathrm{~cm}$ in diameter and .011-0.013 in thick. An external oscillator produces a vibration of the quartz at a frequency of $5 \mathrm{MHz}$. When material is deposited on top of the crystal, its resonance frequency decreases and this change allow us to determine the film thickness through the following relationship: ${ }^{55}$

$$
\text { thickness }=\frac{\rho_{q}}{\rho_{f}} N_{q}\left(\frac{\tau}{\pi R_{z}}\right) \arctan \left(R_{z} \tan \left\{\pi \frac{\tau-\tau_{a}}{\tau}\right\}\right),
$$

where $\rho_{f}$ is the density of the film, $\rho_{q}$ the density of the quartz, $N_{q}$ a crystal frequency constant, $\tau_{q}$ the period of oscillation of the uncoated crystal, $\tau$ the 
period of the coated crystal and $R_{Z}$ the acoustic impedance ratio between the quartz and the deposited film. By knowing these constants the quartz controller can calculate the deposited material thickness and the evaporation rate. These parameters must be loaded to the controller for each different material. Most of the time, the accuracy of the thickness depends on the crystal health. This parameter range from a value of $100 \%$ for a new and uncoated crystal up to $0 \%$ for a total deposited crystal with an extra mass of $25 \mathrm{mg} / \mathrm{cm}^{2}$. In reality, this $0 \%$ should never be reached because in general, the crystals give abnormal readings below $95 \%$.

In the MBE system, one of the crystal monitors is located on top of the target to give a nominal thickens. Another crystal monitor (translator) is placed right below the substrate to measure the actual evaporation rate. With the reading of both crystals we can calibrate the nominal thickness needed to deposit the desired amount of cobalt (4 $\mathrm{nm}$ thick). Typical evaporation rates in the substrate are between $0.05-0.10(\hat{A} / \mathrm{s})$. During the growth, the substrate is rotated to increase the homogeneity of the film and it is held at a fixed temperature of $315{ }^{\circ} \mathrm{C}$ because past experiments probed that cobalt films grown on sapphire (110) at this temperature are smoother. ${ }^{56}$

\subsection{IMAGING OF THE FILM UPON ANNEALING}

The sample is mounted in an Omicron variable temperature sample holder which allows to indirectly heating up the sample by passing a current to a PBN element. The measurement of the temperature is tricky because there is no thermocouple attached to the sample (this might introduce noise in the SPM), also a pyrometer is not useful due to the small size of the sample (the normal size of the pyrometer collecting area of $\sim 1 \mathrm{~cm}^{2}$, whereas the area of our sample is $0.2 \mathrm{~cm}^{2}$ ). Therefore a first estimation of the sample temperature was carried out using a calibration between the power applied to the PBN and the temperature at the sample position (see Figure 27) performed in Omicron Laboratories. ${ }^{57}$ 
The sample was imaged using a non contact atomic force microscope equipped with a needle sensor. A needle sensor is a quartz resonator (see Figure 28) that vibrates vertically at a frequency of $1 \mathrm{MHz}$. The exact value of the resonance frequency is approximately $1 \mathrm{MHz}$ but the real value might change slightly for different tips. Hence the software automatically sweeps the frequency around 1 $\mathrm{MHz}$ to find the precise value for each tip. The excitation voltage used for the tips is approximately $0.4-0.6 \mathrm{mV}$. This voltage determines the amplitude of the tip vibration; typically $0.6 \mathrm{mV}$ is useful for most applications but in cases where the samples are very rough, it is necessary to decrease the voltage to $0.4 \mathrm{mV}$ or even lower. The difference of the applied frequency and the response of the tip determines a difference in phase between the two signals. This phase is constant around the resonance frequency of the needle tip but it decays when the needle is far away from the resonance. The slope of this decay determines the sensitivity of the tips. Typically good tips have a slope between 1 and 3 $\mathrm{deg} / \mathrm{Hz}$; slopes $<1 \mathrm{deg} / \mathrm{Hz}$ indicate very poor or damaged tips. The end of the needle tip has a doped silicon canteliver with an apex radius of $<10 \mathrm{~nm}$. When this canteliver is placed close to a surface, the needle sensor undergoes a phase shift. If the AFM is used in constant phase shift mode, this parameter is used as a feedback to keep the tip always at the same distance from the film. The needle is mounted in a piezoelectric element that moves the tip up or down keeping the phase shift constant. Other piezoelectric elements move the tip in the $X Y$ plane (parallel to the film surface). The voltages necessary to move the tip up/down (for keeping the phase shift constant) in addition to the $X Y$ coordinate of the sample position create the image of the film. The normal phase shift feedback used in the experiments ranged between $-4^{\circ}$ and $-8^{\circ}$. Large negative values of the phase shift makes the tip closer to the film surface allowing better resolution of feature's edges but values that are too high might crash the tip onto the surface. Other parameters used in the experiments are the integral and proportional gains. These parameters control how fast or slow the tip responds to the changes in the morphology in the $Z$ axis during scan. Typical values for 
these gains are $20 \%$ and $10 \%$ for the integral and proportional gain respectively. Higher values for these gains give better spatial resolution (sharper features in the image) but extremely large values generate noise and uncontrolled oscillations of the tip that generate artificial randomly distributed white dots in the images. On the other hand, too small gain parameters might produce tails in the image's features because of a slow feedback response. As a rule of thumb, one should use the smallest gain possible to obtain a good image without increasing very much the noise. These gain parameters depend also on how fast or slow the image is scanned. This is controlled by the scan speed; a slower scan generally results in general better images. In our case, for an image of $2 \times 2 \mu \mathrm{m}^{2}$ a scan speed $<2(\mu \mathrm{m} / \mathrm{s})$ was normally used. Table 4 summarizes the typical scanning parameters for the needle sensor.

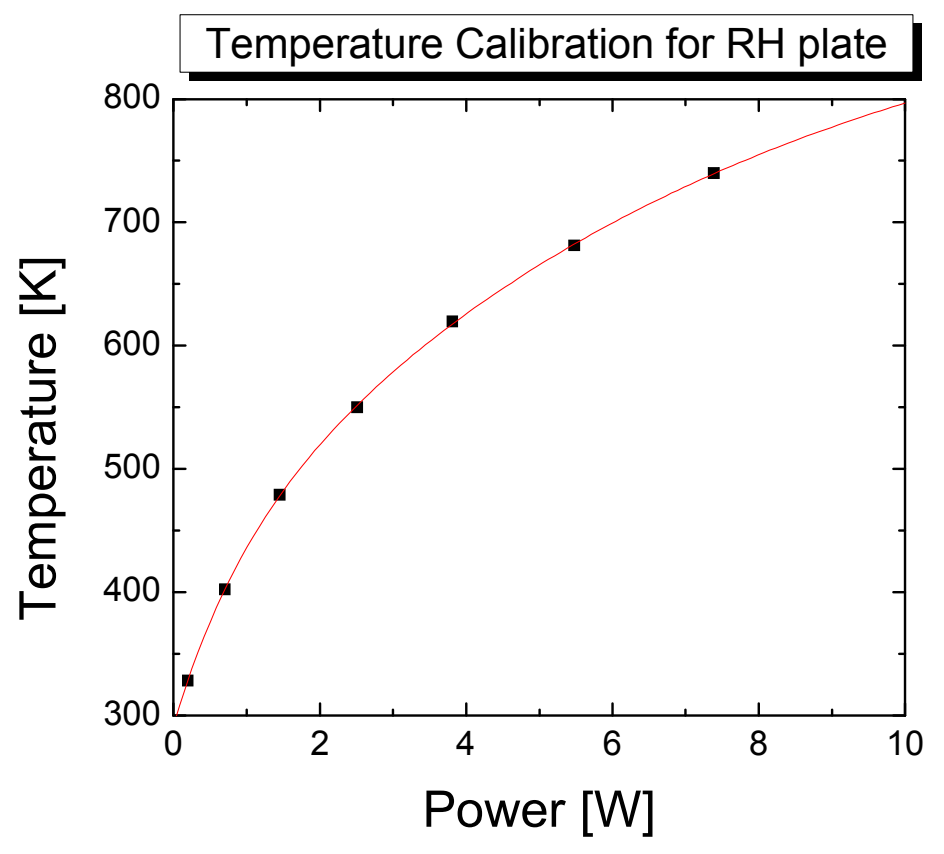

Figure 27. Calibration of temperature for the Omicron indirect heater. The graph depicts the temperature measured in the sample position versus the power applied to the PBN. 
The AFM has to be able to detect very small changes in $Z$ direction (i.e. perpendicular to the surface) when atomic resolution is required (for instance to detect atomic steps in a substrate). In this case the AFM was used with the maximum sensitivity in $Z$ direction; this requires a large $Z$ gain. On the other hand, there are situations when it is necessary to measure large clusters that can be almost $100 \mathrm{~nm}$ tall; in this case a small sensitivity for the $Z$ direction is needed (low $Z$ gain). The Omicron AFM allows controlling the sensitivity of the instrument in the $Z$ axis. The maximum sensitivity is a $Z$ gain of 100 , the default sensitivity is a $Z$ gain of 10 and the lowest sensitivity is a $Z$ gain of 1 . The experiments were carried out using a $Z$ gain of 1 (lowest) in order to measure tall clusters in the film.

\begin{tabular}{|l|l|l|}
\hline $\begin{array}{l}\text { Needle Tip Resonance } \\
\text { Frequency }\end{array}$ & 1 & $\mathrm{MHz}$ \\
\hline Excitation Amplitude & $0.4-0.6$ & $\mathrm{mV}$ \\
\hline Tip Slope & $1-3$ & $\mathrm{Deg} / \mathrm{Hz}$ \\
\hline Phase Shift Feedback & -4 to -8 & $\mathrm{Deg}$ \\
\hline Z Gain & $1-10$ & \\
\hline Integral Gain & $20-30$ & $\%$ \\
\hline Proportional Gain & $10-15$ & $\%$ \\
\hline Scan Speed & $2-3$ & $\mu \mathrm{m} / \mathrm{s}$ \\
\hline
\end{tabular}

Table 4. Typical parameters for the Omicron VT Needle sensor. 


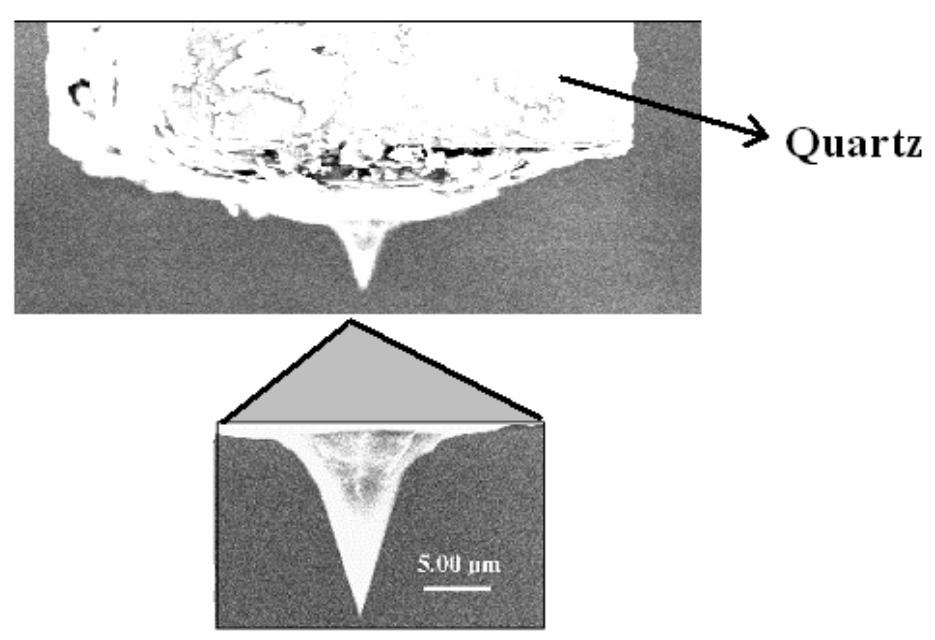

Si Doped Tip

Figure 28. Scanning electron microscopy images of a needle sensor tip.

Before starting the experiment, the needle tip is placed at a scanning distance from the sample. Then, the sample is heated at a rate of $100^{\circ} \mathrm{C}$ per hour up to $450{ }^{\circ} \mathrm{C}$, i.e. just below the cobalt roughening transition. During the annealing process, the pressure in the vacuum chamber was kept below $5 \times 10^{-9}$ Torr. by using a titanium sublimation pump in addition to an ion pump. After reaching 450 ${ }^{\circ} \mathrm{C}$, the power of the PBN heater was increased to obtain the desired temperature above the roughening transition. Once the film reached thermal equilibrium at the temperature set point, the film is imaged as a function of time. The same film area (from $2 \times 2 \mu \mathrm{m}^{2}$ up to $3 \times 3 \mu \mathrm{m}^{2}$ ) is scanned in order to capture the evolution of individual clusters in time.

It is important to mention that a good estimator of the film's thermal equilibrium is the resonance frequency of the needle tip. Figure 29 shows the behavior of the resonance frequency (when the tip is placed at scanning distance from the sample) as a function of the annealing temperature. The resonance frequency does not change from room temperature up to $300{ }^{\circ} \mathrm{C}$ but when the sample temperature is raised from $300{ }^{\circ} \mathrm{C}$ up to $600{ }^{\circ} \mathrm{C}$, the tip's resonance frequency decreases by $17 \%$. Above $600{ }^{\circ} \mathrm{C}$, the needle tip is unstable and it is not possible to obtain good images. The drop of the resonance frequency might be explained 
as a softener of the tip leading to a smaller spring constant and therefore to a smaller resonance frequency. In any case, after the temperature of the tip was set in the range from $300^{\circ} \mathrm{C}$ to $600^{\circ} \mathrm{C}$, the resonance frequency will change for some time, but eventually it will stabilize and it will remain constant until the temperature is changed again. A resonance frequency change less than $2 \mathrm{~Hz}$ in 20 minutes is an indication that the film and tip system have reached thermal equilibrium.

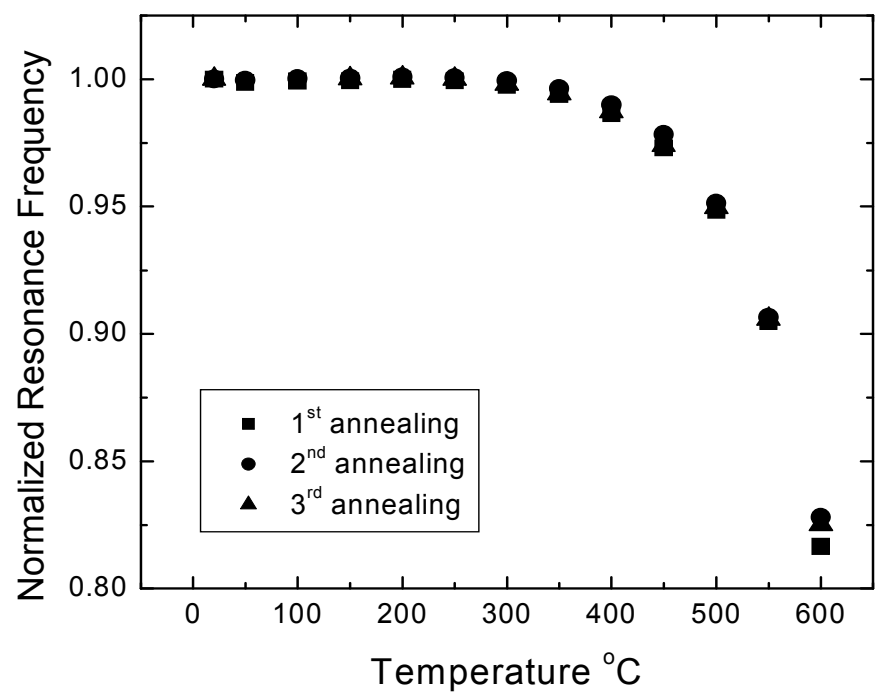

Figure 29. Variation of the resonance frequency for the needle tip at different temperatures. We can see the reproducibility of the resonance change for 3 different annealing experiments. 


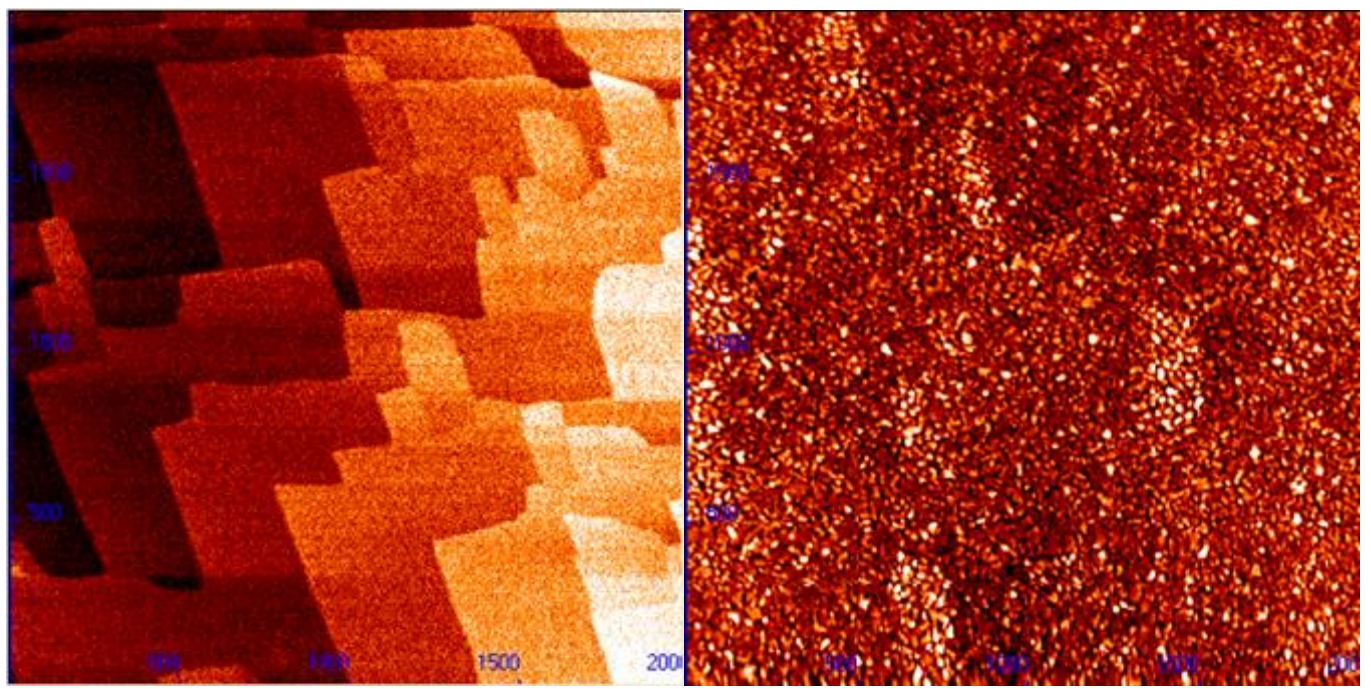

(a)

(b)

Figure 30. (a) Sapphire (11-20) before growth. (b) $4 \mathrm{~nm}$ thick cobalt film immediately after growth. The image sizes are $2 \times 2 \mu \mathrm{m}^{2}$.

\section{RESULTS}

\subsection{MORPHOLOGY}

Prior the experiment, the sapphire substrate was imaged using AFM before the evaporation of cobalt as shown in Figure 30(a). The irregular patterns in the surface are atomic terraces formed during the annealing in air at $1400{ }^{\circ} \mathrm{C}$. The difference in height between terraces corresponds to atomic steps of $0.2 \mathrm{~nm}$. The observation of atomic steps in the sapphire is a good indication of a clean substrate. After imaging the clean sapphire substrate, it was transported to the MBE chamber to grow $4 \mathrm{~nm}$ of cobalt. Figure $30(\mathrm{~b})$ is an image of the cobalt film 
(4 $\mathrm{nm}$ thick) immediately after deposition. The cobalt surface after growth was relatively smooth with a roughness of $0.7 \mathrm{~nm}$.

After imaging the sapphire and the cobalt film, the sample was heated up and the film was scanned during the annealing. In-situ AFM images were taken at different temperatures for long periods of time. Figure 31 shows the images for a cobalt film of $4 \mathrm{~nm}$ annealed at a fix temperature of $535^{\circ} \mathrm{C}$ for several hours. The same area was scanned during all the annealing processes in order to track the changes of morphology of individual clusters. Each image scan took approximately 15 minutes; therefore we were limited by this amount of time to observe morphology changes in the film. The annealing time was defined as the time elapsed between when the film reaches thermal equilibrium (estimated with the needle sensor resonance frequency) and the instant that the image scan was finished. The first image in Figure 31 corresponds to the film annealed at $535{ }^{\circ} \mathrm{C}$ for 0.5 hours. Even at this early stage of the annealing the film looks very different from the image taken before annealing. At 0.5 hours the image shows the formation of white and black irregular dots randomly distributed in the film. In order to understand these structures it is important to note that the features of light red or white dots represent mountains on the surface of the film whereas black dots or very dark red sports represent pits or depressions in the film. These islands and depressions have a height of a few tenths of $\mathrm{nm}$ and diameters of up to $100 \mathrm{~nm}$. These observations prove that the film has already undergone a roughening transition which is consistent with previous observations showing the transition taking place at $459^{\circ} \mathrm{C} .{ }^{21}$ At 0.8 hours a large island has nucleated on the bottom left hand side of the image (cluster 1) with a diameter of $\sim 500 \mathrm{~nm}$ and an average height of $40 \mathrm{~nm}$. This structure was not present in the previous image acquired 15 minutes before. This would indicate that the process of nucleation is somewhat faster than 15 minutes and that our scanning process is limited to the observation of the late stages of cluster growth. 
After 2 hours of annealing, another large island nucleated (cluster 2) below cluster 1 . This new island had a diameter of $\sim 500 \mathrm{~nm}$ and an average height of $15 \mathrm{~nm}$. Cluster 1 continued to grow in diameter and at this stage its average height was $45 \mathrm{~nm}$. Also at $2 \mathrm{hrs}$ vacancy islands (black dots) with average diameters of a few tens of $\mathrm{nm}$ up to $100 \mathrm{~nm}$ were observed. However, these holes apparently disappeared in the image taken after 3 hours of annealing, leading only to the development of the existing clusters and to the nucleation of

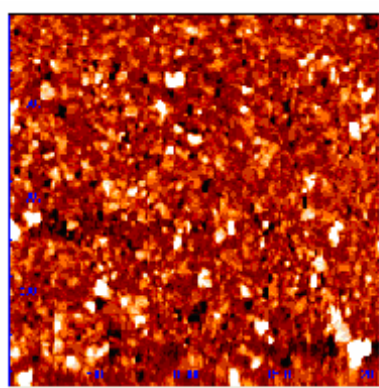

0.5 h

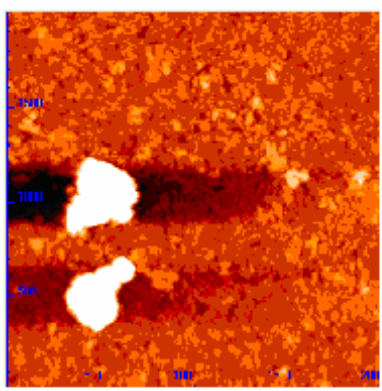

3 h

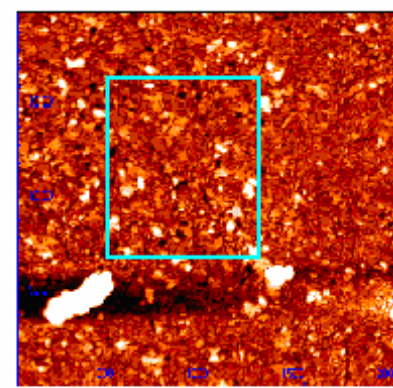

0.8 h

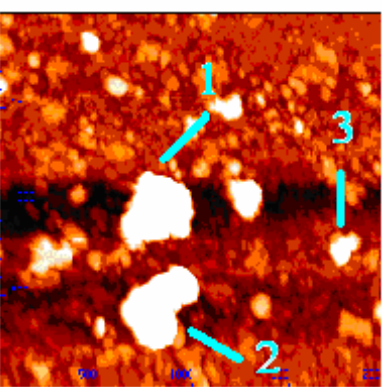

$7.5 \mathrm{~h}$
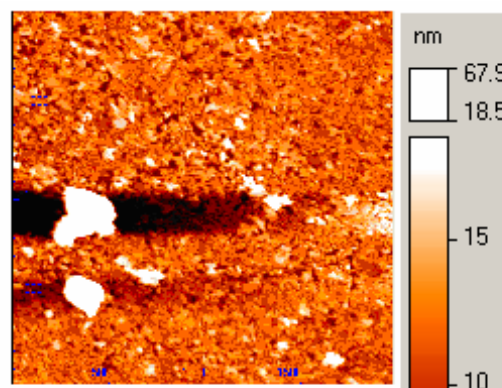

2 h

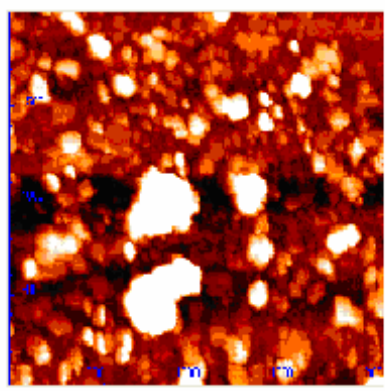

$16 \mathrm{~h}$

Figure 31. AFM images $\left(2 \times 2 \mu \mathrm{m}^{2}\right)$ of a cobalt film (4 nm thick) on $\mathrm{Al}_{2} \mathrm{O}_{3}$ (110) annealed for 16 hours at $535^{\circ} \mathrm{C}$. The blue square in the $0.8 \mathrm{hr}$ image is the region used for computing the roughness of the film.

new ones. After 3 hours the diameter and height of cluster 1 did not change very much, unlike cluster 2, which continued growing in diameter (700-800 nm) and height $(30 \mathrm{~nm})$. In the next images taken at $7.5 \mathrm{hrs}$ and $16 \mathrm{hrs}$ few morphological changes were observed for clusters 1 and 2, but the whole film continued to roughen and many small clusters nucleated at late stages of annealing. It is important to note that after 16 hours of annealing the film did not show signs of de-wetting. 
Figure 32 shows the evolution of another $4 \mathrm{~nm}$ thick film annealed at $565^{\circ} \mathrm{C}$ for over $11 \mathrm{hrs}$. The same area of the film was tracked during the annealing process. At $t=0 \mathrm{hr}$ the film already showed a large number of clusters with heights of $40 \mathrm{~nm}$ and diameters of approximately $250 \mathrm{~nm}$. This result is interesting considering that at a temperature of $535^{\circ} \mathrm{C}$ the number of nucleated clusters and their average height was much smaller even after 16 hours of annealing. For $2.6 \mathrm{hr}$ and $9.4 \mathrm{hr}$ the roughening process continued and the clusters became even taller, with some of them being $\sim 100 \mathrm{~nm}$ in height. Even though the roughening process seemed to occur much faster than at the annealing temperature of $535{ }^{\circ} \mathrm{C}$, the behavior of the island's diameter at $565{ }^{\circ} \mathrm{C}$ resembles the dynamics at $535^{\circ} \mathrm{C}$ because from $2.6 \mathrm{hr}$ up to $9.4 \mathrm{hr}$ the clusters' area seems almost unchanged. The area and height dynamics will be analyzed in more detail in the following sections.

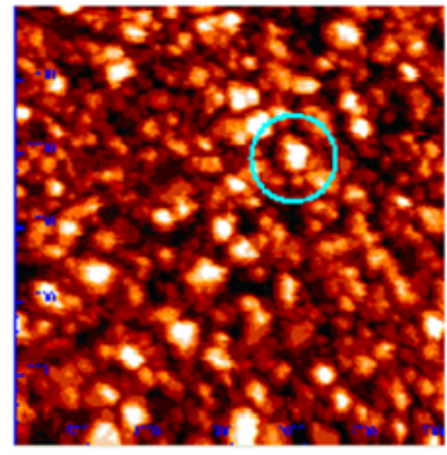

$\mathbf{t}=\mathbf{0} \mathbf{h}$

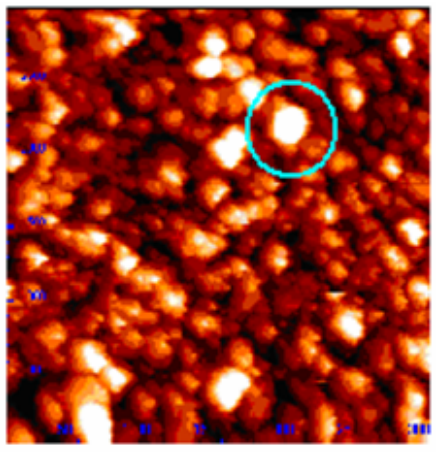

$\mathbf{t}=2.6 \mathrm{~h}$

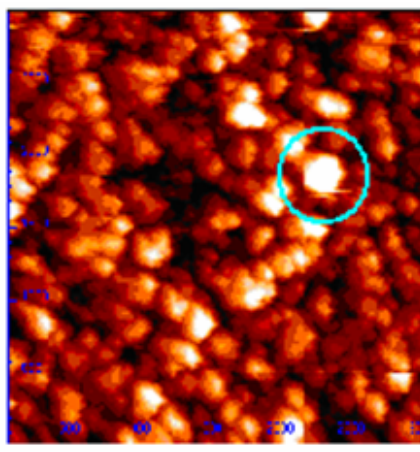

$\mathbf{t}=9.4 \mathrm{~h}$

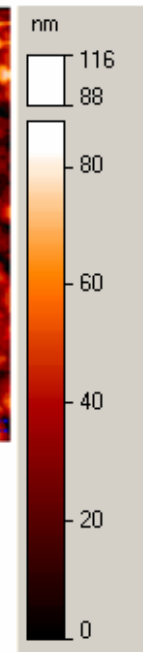

Figure 32. AFM images of a cobalt film annealed at $565{ }^{\circ} \mathrm{C}$ for several hours. Image sizes are $\left(3 \times 3 \mu \mathrm{m}^{2}\right)$.

Figure 33 shows the time evolution of another cobalt film ( $4 \mathrm{~nm}$ thick) annealed at a temperature of $590^{\circ} \mathrm{C}$. In this case a rapid roughening and rupture of the film was observed. After $1 \mathrm{hr}$ of annealing, the AFM image shows the formation of large clusters and pits on the cobalt film. After $7 \mathrm{hrs}$ of annealing, the pits 
continued to grow in diameter and started to coalesce with neighboring vacancy

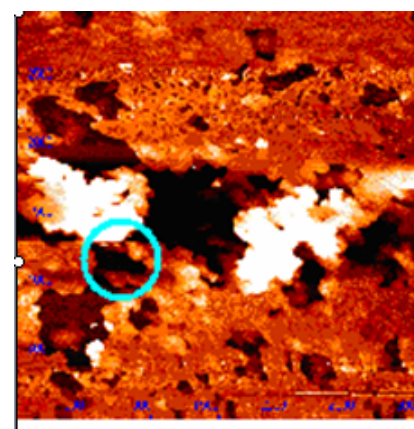

1 hr

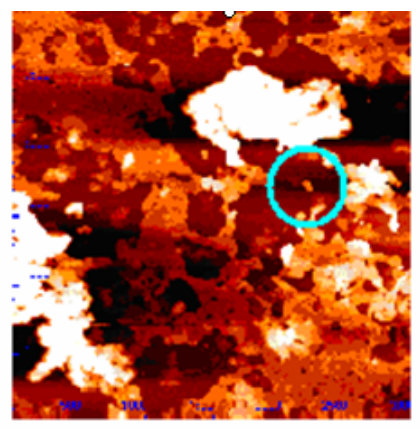

$3 \mathbf{~ h r}$

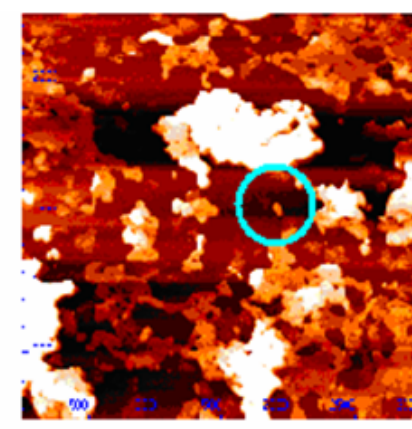

$7 \mathbf{h r}$

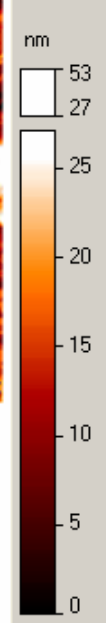

Figure 33. AFM image $\left(3 \times 3 \mu \mathrm{m}^{2}\right)$ of cobalt film on sapphire (110) annealed at a constant temperature of $590{ }^{\circ} \mathrm{C}$. De-wetting of the film is observed at this temperature.

islands, resulting in what looks like a de-wetting transition. The vacancy islands have an irregular shape and no rim is observed on their perimeter. Rims have been observed in intermediate stages of polymer de-wetting ${ }^{35}$ and their formation is explained by the mass conservation principle, which requires that the material removed to form the vacancy island must form the rim material. Another characteristic of the vacancy islands in the Co films is that their inner portion was not completely empty. This phenomenon also has been observed in de-wetting of polymers ${ }^{51,58}$ and is believe to be related to inhomogenities of the film or substrate. Impurities or defects in the film or substrate might activate the nucleation of vacancy islands during the early stages of the de-wetting process.

It is also instructive to study the AFM images in three dimensions. Figure 34 shows a set of three-dimensional images taken at different annealing temperatures and times. In Figure 34 (a) cluster 1 in Figure 31 is apparent on the lower left corner of the image. Cluster 1 has a flat top when it started growing (Figure 34(a)-(b)) and as time increased the cluster grew in height and became 
sharper (Figure $34(\mathrm{e})-(\mathrm{f})$ ). Cluster 2 first appears in Figure 34(c), and it is apparent that at the beginning the island had a flat top that became sharper at

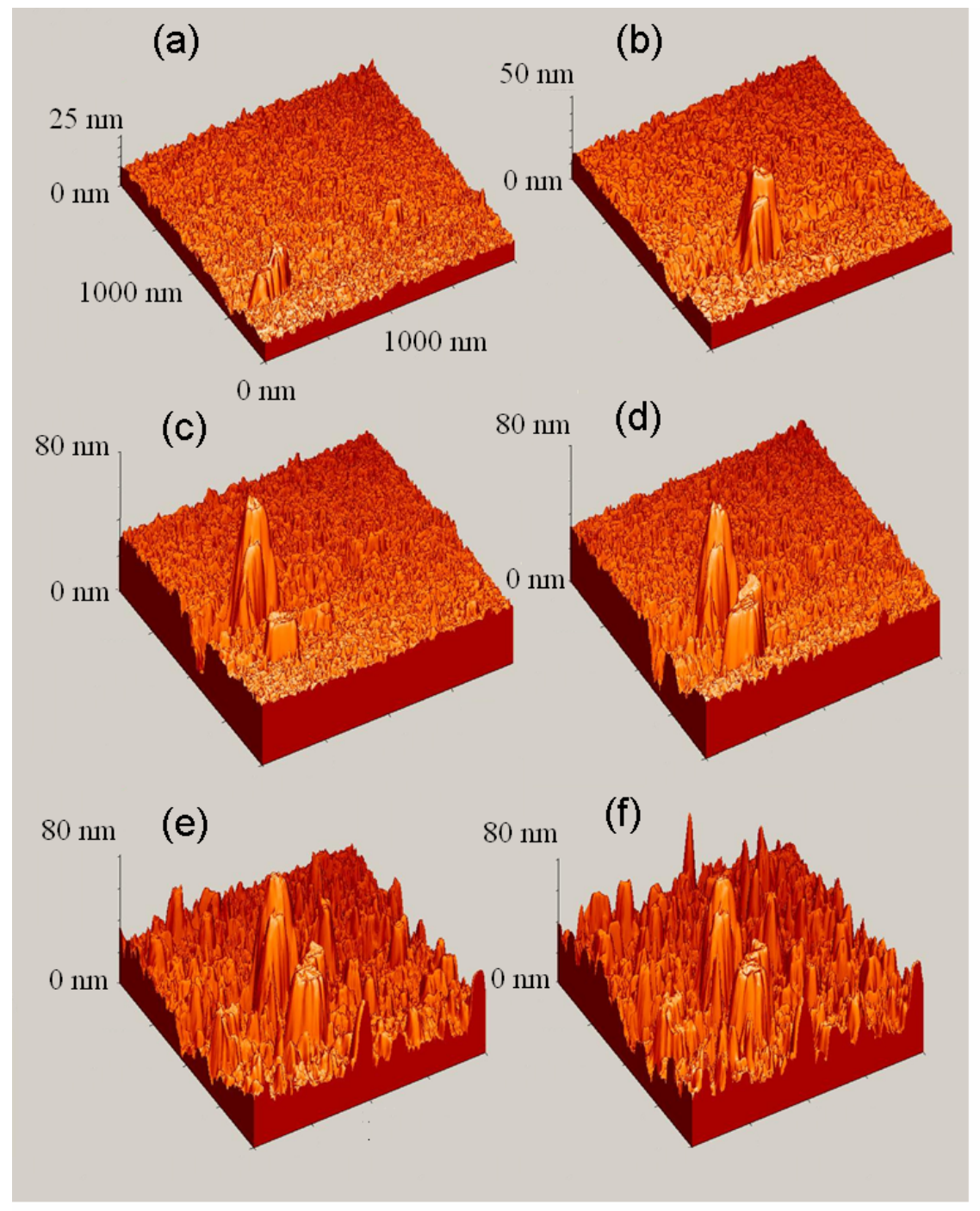

Figure 34. 3D images for cobalt film annealed at $535{ }^{\circ} \mathrm{C}$ at different annealing times. The size of the images is $2 \times 2 \mu \mathrm{m}^{2}$. The annealing time is relative to the nucleation of the first cluster in image (a). Annealing times: (a) $0.8 \mathrm{hr}$, (b) $1.4 \mathrm{hr}$, (c) $2.0 \mathrm{hr}$, (d) $3.0 \mathrm{hr}$, (e) $10 \mathrm{hr}$ and (f) $16 \mathrm{hr}$.

later annealing times. 
The generation of this flat top is a general behavior of the islands at this annealing temperature. In contrast, annealing the sample at $565^{\circ} \mathrm{C}$ (Figure 35) produces islands with sharp tips instead of flat tops. Although this behavior might seem to contradict what was observed in the film annealed at $535^{\circ} \mathrm{C}$, this can be understood by comparing Figure 31(f) with Figure 35(a). These two images are very much alike, that is, in both images there are a large number of clusters, many of them with sharp ends. Therefore, the morphology of a film annealed at a low temperature for a long time resembles the behavior of a film annealed at a higher temperature for a shorter time.

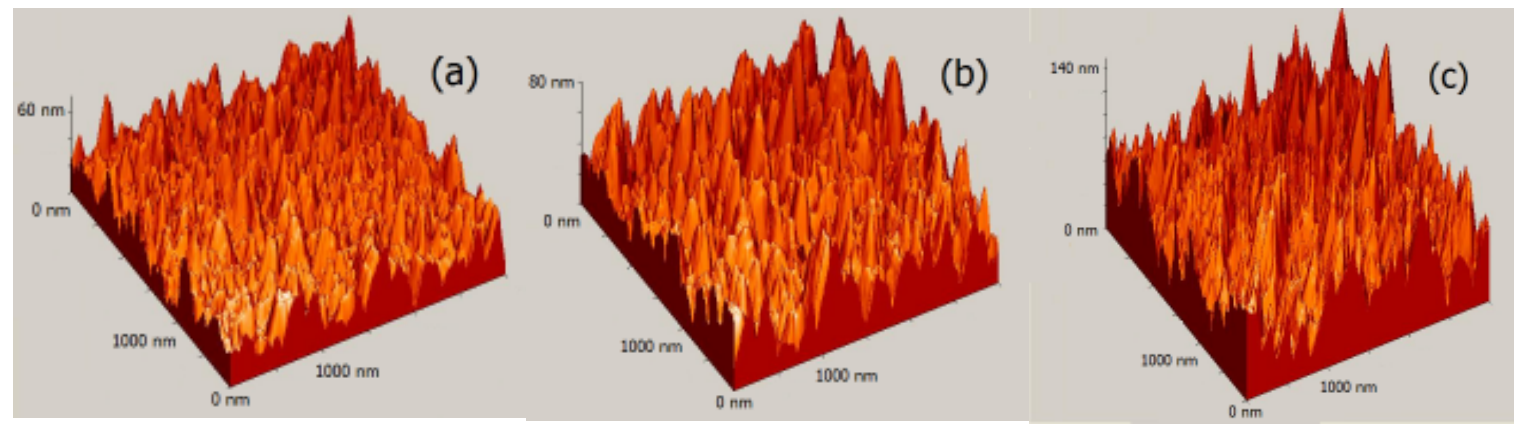

Figure 35. 3D images of cobalt film annealed at $565^{\circ} \mathrm{C}$ at different annealing times. The image sizes are $3 \times 3 \mu^{2}$. Annealing times: (a) $1.7 \mathrm{hr}$, (b) $2.4 \mathrm{hr}$ and (c) $8.7 \mathrm{hr}$.

\subsection{FILM ROUGHNESS}

The AFM and STM images were analyzed quantitatively by measuring their roughness defined as

$$
\rho=\left(\frac{1}{M N} \sum_{i=0}^{M-1} \sum_{j=0}^{N-1}\left(h_{i j}-<H>\right)^{2}\right)^{1 / 2},
$$

where $\mathrm{M}$ and $\mathrm{N}$ are the size of the image in pixels, $h_{i j}$ is the height of the image at a pixel position $(i, j)$ and $\langle H\rangle$ is the average height of the image. Figure 36 shows the roughness as a function of time for one cobalt film (4 $\mathrm{nm}$ thick) annealed at $535{ }^{\circ} \mathrm{C}$ (red dots). The roughness was computed using Equation 
(87) for the full image size. The time was defined as the relative annealing time from the time when the film reached thermal equilibrium. The roughness at 535 ${ }^{\circ} \mathrm{C}$ increased abruptly for annealing times $<3 \mathrm{hr}$ and then continued increasing at a slower rate at later times, reaching more than $10 \mathrm{~nm}$ after approximately $16 \mathrm{hr}$. In Figure 36 the red line is a fit of the data to a power law

$$
\rho=\rho_{o}\left(t-t_{o}\right)^{\alpha},
$$

where $t$ is the absolute annealing time, $t_{0}$ corresponding to an effective initial annealing time, $\rho_{o}$ is a scaling factor, and $\alpha$ an exponent. Both parameters and their errors are obtained by fitting equation (88) to the experimental data. On the other hand, $t_{0}$ was estimated as the difference between the time that the first image was obtained at $535{ }^{\circ} \mathrm{C}$ and the time when the film reached thermal equilibrium. Because it takes approximately 1 hour to reach thermal equilibrium and the film morphology may have changed during that interval of time, we estimate the uncertainty of $t_{0}$ to be one $\mathrm{hr}$. A fit to the data excluding the first 3 hrs of annealing resulted in $\alpha=0.20$ (see table Table 5). Of course this result is greatly influenced by the nucleation of clusters 1 and 2 and its statistical significance is poor. In order to analyze the roughness of the film excluding those clusters, the roughness in the rectangular area outlined in Figure 31 was computed separately; the results are shown in Figure 37. In this case, the roughness increased linearly with increasing annealing time without signs of saturation. This might be related to the fact that the roughness is defined as the rms value of the surface height inside an area. In that region, some clusters develop earlier than others, and once these reach maximum ripening, others are just starting to grow. Therefore, at this temperature it could take several hours or even days to the roughness to reach saturation.

Figure 38 shows the roughness as a function of time for another cobalt film annealed at $565{ }^{\circ} \mathrm{C}$. At this temperature the film becomes rough more homogeneously than at $535^{\circ} \mathrm{C}$ because clusters grow simultaneously, as seen in Figure 35; hence in this case the roughness was computed for the entire image. The roughness also increased faster for short annealing times $(<3 \mathrm{hrs})$ and 
slower for the later annealing stages, but in this case the roughness climbed to $20 \mathrm{~nm}$ after 10 hours of annealing at $565^{\circ} \mathrm{C}$, compared with only $10 \mathrm{~nm}$ after 16 $\mathrm{hr}$. of annealing at a lower temperature of $535{ }^{\circ} \mathrm{C}$. This result is understandable because at higher annealing temperatures the roughening process is expected to be faster than at lower temperature due to the acceleration of thermodynamic processes. This phenomenon can be observed by inspecting Figure 34 (f) and Figure 35 (a). If the two large clusters are neglected on Figure 34 (f), the film annealed at $535{ }^{\circ} \mathrm{C}$ for 16 hours looks very similar to the film annealed at $565{ }^{\circ} \mathrm{C}$ for only 1.7 hours. A fit to the $565{ }^{\circ} \mathrm{C}$ data excluding the first 3 hours of annealing using the power law in Equation 2 (red line) yielded an exponent $\alpha=$ 0.21 , which is very close to the exponent $\alpha=0.20$ computed for $535{ }^{\circ} \mathrm{C}$. The roughness as a function of time for the annealing temperature of $590^{\circ} \mathrm{C}$ can be observed on Figure 39. Roughness increases faster the first 3 hours and much slower after that yielding to an exponent $\alpha=0.16$ (see Table 5). This is the smallest exponent of the three annealing temperatures. The reason for this might be that clusters in the film start to saturate at this higher temperature producing a slower power law growth. 


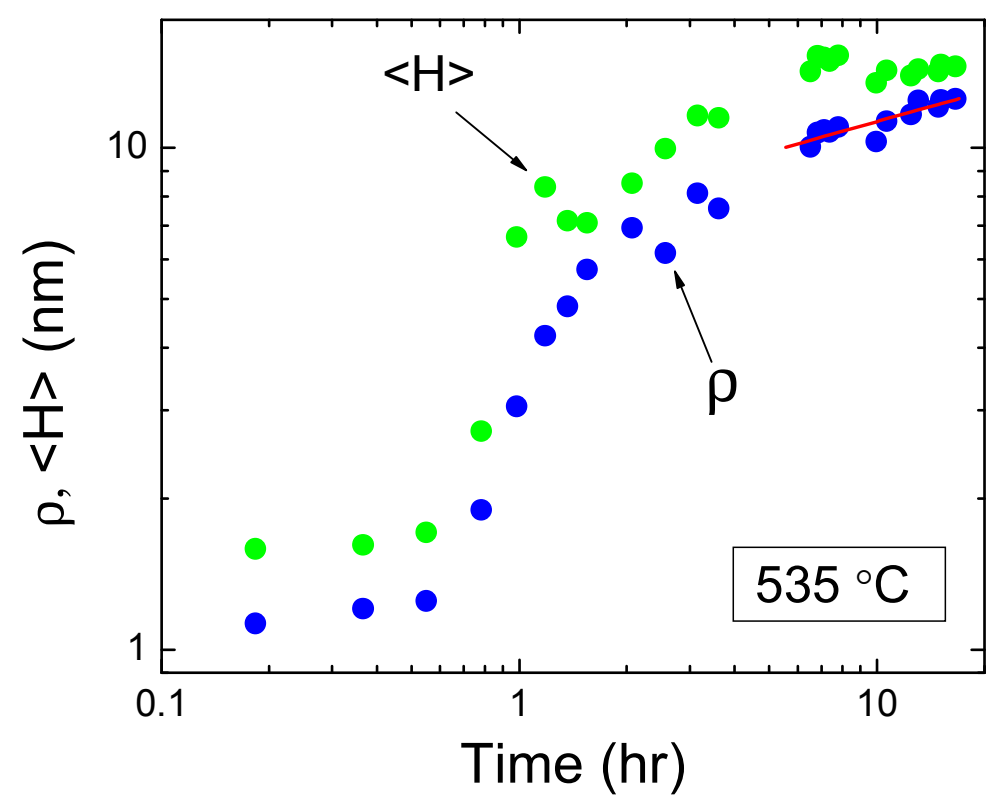

Figure 36. Roughness as a function of time (red dots) for the annealed films at $\mathrm{T}=535{ }^{\circ} \mathrm{C}$ and $\mathrm{T}=565{ }^{\circ} \mathrm{C}$. Blue dots represent the average height of the image intensity. The red line depicts the fit to Equation (88).

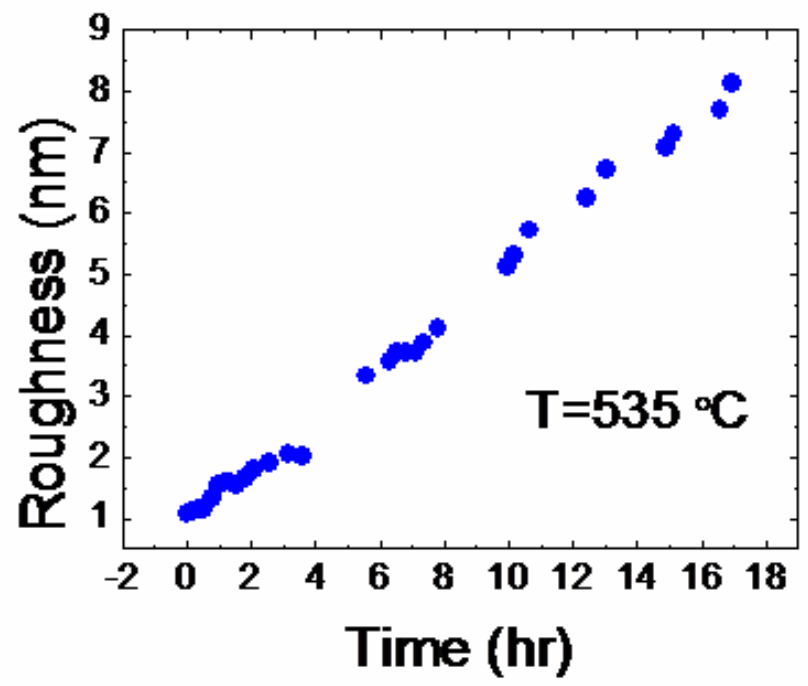

Figure 37. Roughness of cobalt film annealed at $535^{\circ} \mathrm{C}$. The roughness was computed excluding the clusters 1 and 2 from the images. 
Finally, the average image height $\langle H\rangle$ was calculated for the three annealing temperatures as a function of time (see green dots on Figure 36, Figure 38 and Figure 39). The $\langle H\rangle$ was calculated using the histogram of heights for each image (obtained from the acquisition software of the Omicron SPM) and the relationship

$$
<H>=\frac{\sum_{i} f_{i} h_{i}}{\sum_{i} f_{i}},
$$

where $f_{i}$ is the count frequency of some determined height $h_{i}$ in the image. Surprisingly, the average height of the images increased up to $15 \mathrm{~nm}$ for $535^{\circ} \mathrm{C}$, $25 \mathrm{~nm}$ for $565{ }^{\circ} \mathrm{C}$ and more than $30 \mathrm{~nm}$ for $590{ }^{\circ} \mathrm{C}$, even though the nominal thickness of the as-grown film was approximately only $4 \mathrm{~nm}$ thick. We would have expected $\langle H>$ to fluctuate around $4 \mathrm{~nm}$ independently of annealing time and roughness. However, $<H>$ apparently grows with time following the same dependence of the roughness on time. The explanation for this behavior might be simply that the data acquisition software labels arbitrarily the zero level of intensity (height offset) for each image producing an apparent thickening of the film. However, it is also true that the time dependence is similar to the roughening process and therefore there might be a more fundamental physical explanation for this behavior. For instance, the film thickening might be due the formation of holes inside the film or interdifusion between the cobalt film and aluminum from the substrate. These questions will be addressed in more detail in the discussion section. 


\begin{tabular}{c|ccc}
\hline $\begin{array}{c}\text { Temperature } \\
{ }^{\circ} \mathrm{C}\end{array}$ & $\begin{array}{c}\rho_{0} \\
(\mathrm{~nm})\end{array}$ & $\begin{array}{c}\mathrm{t}_{0} \\
(\mathrm{hr})\end{array}$ & $\alpha$ \\
\hline 535 & $1.1 \pm 7.1$ & $0.15 \pm 1.00$ & $0.20 \pm 0.03$ \\
565 & $12.3 \pm 0.2$ & $0.86 \pm 1.00$ & $0.21 \pm 0.01$ \\
590 & $7.2 \pm 4.6$ & $-1.32 \pm 1.30$ & $0.16 \pm 0.24$ \\
\hline
\end{tabular}

Table 5. Fitting parameters for roughening experiments.

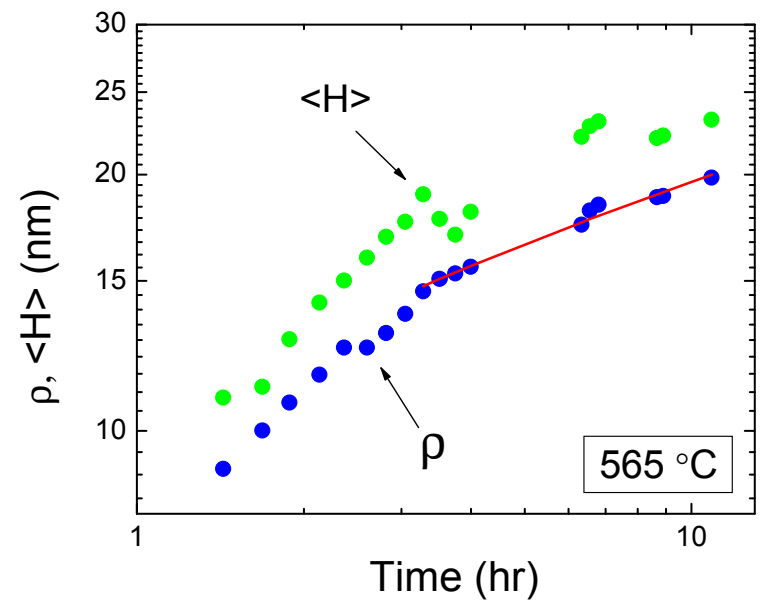

Figure 38. Roughness as a function of time (blue dots) for the film annealed at $565^{\circ} \mathrm{C}$. The red line depicts the fitting of the data with equation (88). Green dots depict the average height of the film. 


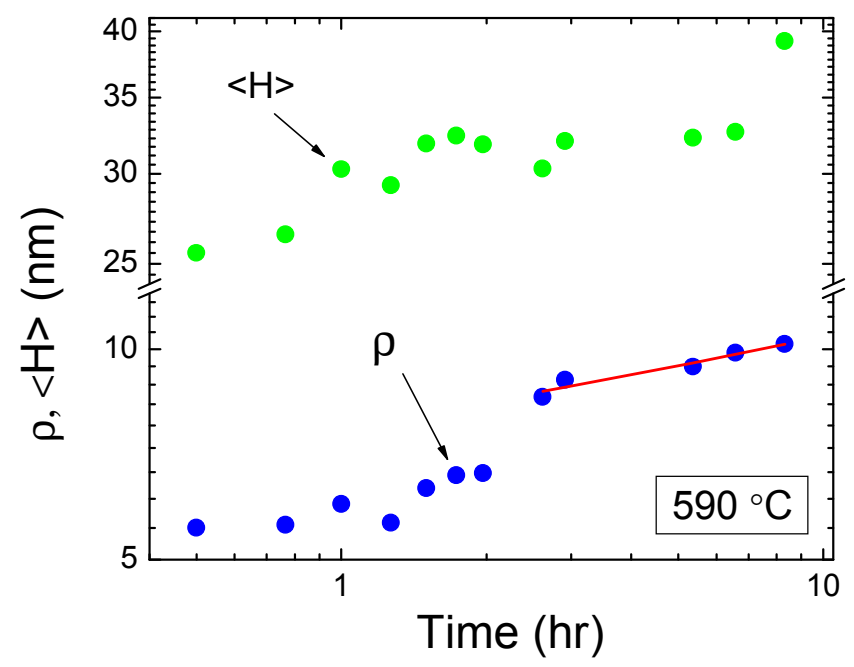

Figure 39. Roughness as a function of time (blue dots) for the film annealed at $590{ }^{\circ} \mathrm{C}$. The red line depicts the fitting of the data with equation (88). Green dots represent the average height of the film.

\subsection{HEIGHT OF CLUSTERS}

The height of three clusters was computed for the film annealed at $535^{\circ} \mathrm{C}$ as a function of time. Figure 40 shows an AFM image with light blue circles indicating the clusters considered in the calculations. The blue line that crosses cluster 2 depicts the direction of the line profile plotted at the bottom of Figure 40 . The line profile of cluster 2 shows that the top of the island is flat but irregular and also that the background around the island is not constant. Therefore, to determine the island's height, the difference between the island top and the background (vertical blue lines in line profile) was computed in different positions of the island's top and the background around the cluster. More than 15 averages were considered for computing the height of a single cluster. Figure 41 depicts the cluster's height as a function of time for the three clusters. The time $t=0$ in the graphs is relative to the instant when cluster 1 was first observed in the images. Cluster 1 appeared first while cluster 3 appeared last. The clusters' height 
increased as a function of time, a fact that was already evident in the roughness analysis. The clusters also achieved over $90 \%$ of their maximum height within the first 3 hours of annealing, growing at a much slower rate after that. Another observation is that the largest clusters are those that develop earlier in the annealing process; for example, cluster 1 develops at the beginning of the annealing process and reaches a maximum average height of $\sim 60(\mathrm{~nm})$, whereas clusters 2 and 3 , which appear at 2 hours and 4.5 hours later, respectively, reach heights of only $45 \mathrm{~nm}$ and $30 \mathrm{~nm}$, respectively. One explanation for these phenomena might be related with the amount of cobalt available to create these clusters. Early clusters have more material nearby and therefore they can grow taller. 

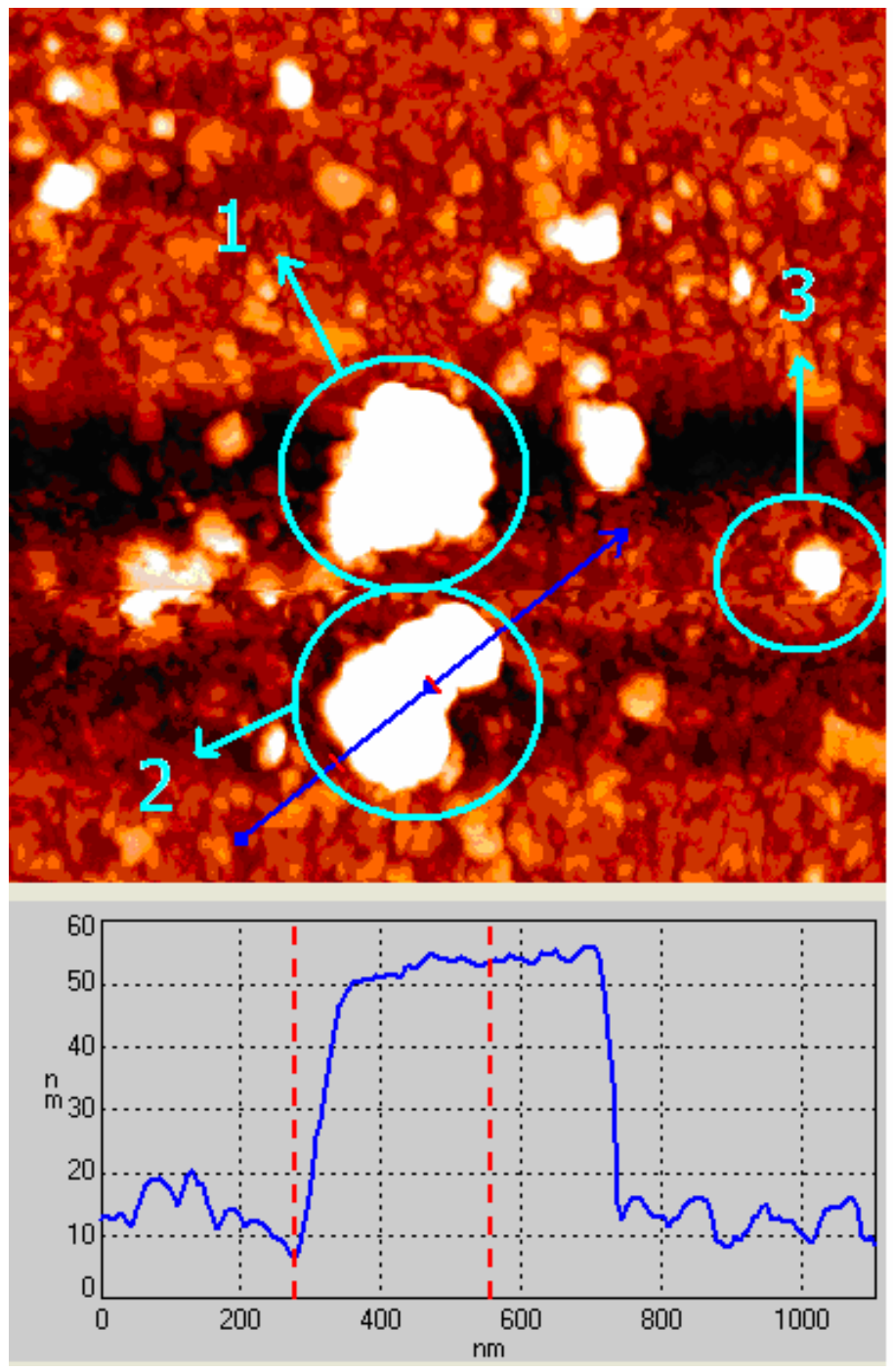

Figure 40. AFM image $\left(2 \times 2 \mu \mathrm{m}^{2}\right)$ of cobalt film annealed at $535^{\circ} \mathrm{C}$ for 7.5 $\mathrm{hr}$. The bottom plot represents the section of the blue arrow in the AFM image. The cluster height was measured in the island enclosed by light blue circles.

As mentioned before (see Figure 41), it is easy to distinguish two regimes of cluster growth for the film annealed at $535^{\circ} \mathrm{C}$. For short annealing times $(\mathrm{t}<2$ $\mathrm{hr}$ ), the cluster's height increased very quickly but for long annealing times $(t>2$ $\mathrm{hr}$ ) the clusters' height increased at a much slower rate. In order to determine if 


\begin{tabular}{c|ccc}
\hline Cluster & $\mathrm{H}(\mathrm{nm})$ & $\mathrm{t}_{0}(\mathrm{hr})$ & $\zeta\left(\mathrm{hr}^{-1}\right)$ \\
\hline 1 & $58.39 \pm 0.71$ & $0.23 \pm 0.02$ & $1.17 \pm 0.08$ \\
2 & $43.94 \pm 0.59$ & $0.95 \pm 0.06$ & $0.52 \pm 0.04$ \\
3 & $26.04 \pm 0.31$ & $4.36 \pm 0.03$ & $0.92 \pm 0.05$ \\
\hline
\end{tabular}

Table 6. Fitting parameters of the exponential clusters' growth for 3 different islands annealed at $535^{\circ} \mathrm{C}$.

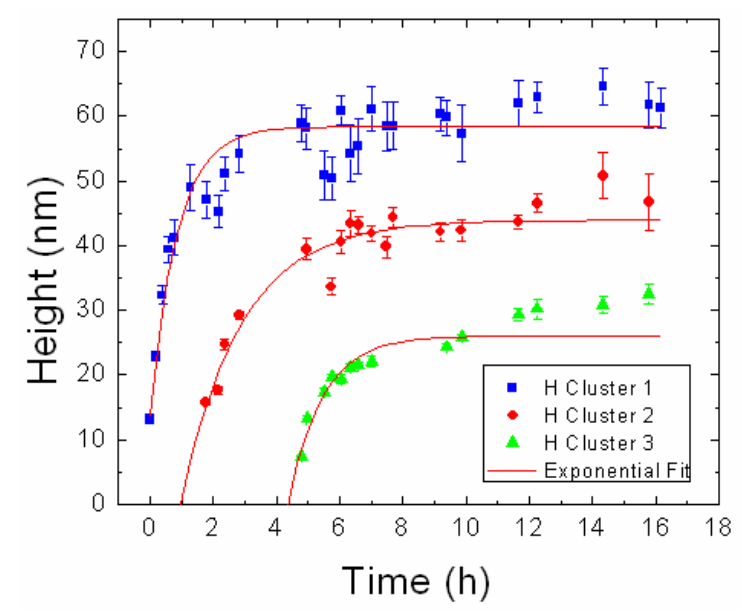

Figure 41. Height as a function of time for 3 different clusters annealed at $535^{\circ} \mathrm{C}$. Red solid line depicts the exponential fitting to the experimental data.

the cluster's growth at short annealing times is consistent with surface instabilities, the exponential growth expression

$$
h=H\left\{1-\exp \left(-\varsigma\left(t-t_{0}\right)\right)\right\}
$$

was fitted to the experimental data, where $H$ is the height of the cluster when time goes to infinity; $t_{0}$ is the absolute time when the cluster start growing, $t$ is any arbitrary time and $\zeta$ a characteristic frequency of the cluster's growth. 

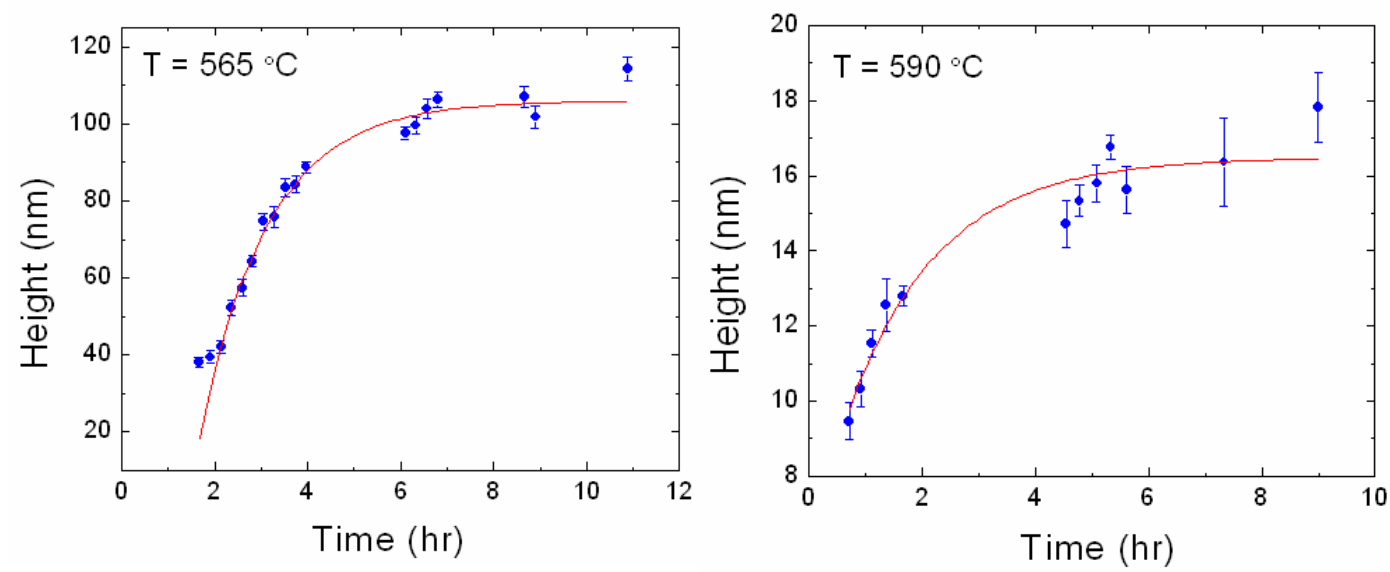

Figure 42. Growth of the cluster height with time for 2 different annealing temperatures. The red line depicts the exponential fitting to the experimental data. The exponential fitting at $565{ }^{\circ} \mathrm{C}$ excludes the first 2 data points.

\begin{tabular}{c|ccc}
\hline Temperature & $\mathrm{H}(\mathrm{nm})$ & $\mathrm{t}_{\mathrm{o}}(\mathrm{hr})$ & $\zeta\left(\mathrm{hr}^{-1}\right)$ \\
\hline $565^{\circ} \mathrm{C}$ & $106.05 \pm 1.20$ & $1.39 \pm 0.06$ & $0.68 \pm 0.04$ \\
$590^{\circ} \mathrm{C}$ & $16.49 \pm 0.36$ & $-0.74 \pm 0.34$ & $0.62 \pm 0.13$ \\
\hline
\end{tabular}

Table 7. Fitting parameters of a exponential function to the clusters' height as a function of time.

The fitting parameters of the exponential curve to the experimental data can be observed in Table 6 . It can be appreciated in Figure 41 that the exponential growth fits well for short annealing times for all three clusters annealed at $535^{\circ} \mathrm{C}$; however, for long annealing times, the exponential growth seems not to fit well the experimental data because the clusters do not saturate and continue growing during the experiment. This effect is not so evident in the largest clusters ( 1 and 2 ) but in the smallest one (cluster 3 ) there is a significant deviation for longer times. The same fitting was carried out for clusters on films annealed at $565{ }^{\circ} \mathrm{C}$ and $590^{\circ} \mathrm{C}$ (see Figure 42). There the exponential curve fits extremely well for short and long times for the cluster annealed at $565{ }^{\circ} \mathrm{C}$ but at $590{ }^{\circ} \mathrm{C}$ the 
exponential curve does not fit well the data for either long and short times, which can be appreciated through the large percent of error in $t_{0}$ and $\zeta$ of $46 \%$ and $21 \%$, respectively. This might be due to the fact that the cluster already exists in the film when tracking was started and therefore its behavior is only observed at later times. However, it is possible to conclude through the clusters studied previously that the exponential growth seems to be best on describing the cluster evolution for short annealing times which is consistent with surface instabilities of the film, but apparently another mechanism dominates for long annealing times.

In order to analyze the behavior of the clusters for long times, power law fittings were carried out on the clusters. The red lines in Figures 10 (b), (c) and (d) are power law fits to the experimental data excluding the data for the first two hours. The power law is similar to that of Equation 2, namely

$$
h=h_{o}\left(t-t_{o}\right)^{\beta} \text {, }
$$

where $h$ is the height as a function of time $t, h_{0}$ is a characteristic height, $t_{o}$ is an effective initial time, and $\beta$ is an exponent. Both, $h_{0}$ and $\beta$ are fitting parameters of equation (89) to the experimental data. In this case $t_{0}$ was estimated by inspection of the images as the time immediately before the formation of the cluster in the film. The data fit the power law dependence well (see Table 8); the results of the fits were: for cluster $1, \beta=0.16$, for cluster $2, \beta=0.22$, and for cluster $3, \beta=0.24$. The exponent for cluster 3 was very close to $\beta=0.25$, which is consistent of a diffusion limited mechanism for cluster growth as described by the $3 \mathrm{D} / 2 \mathrm{D}$ model. This remained true even at higher annealing temperatures. Figure 44 shows the cluster's height as a function of time for a fixed annealed temperature of $565^{\circ} \mathrm{C}$. The cluster studied in this case is the island circled in light blue in Figure 32. The height of the island increased rapidly for annealing times $<3 \mathrm{hr}$ and slower for times $>3 \mathrm{hr}$ as observed at $\mathrm{T}=535^{\circ} \mathrm{C}$. These data can also be fitted with a power law (Equation 3 ) for $t>3 \mathrm{hr}$ yielding $\beta=0.25$. Finally, for the film annealed at $590{ }^{\circ} \mathrm{C}$ a small island (light blue circle in Figure 
33) was tracked after it de-attached from the film. The height of the island was measured as a function of time as illustrated on Figure 45. The height increased quickly for $t<1.5 \mathrm{hrs}$ and then more slowly after $\sim 1.5 \mathrm{hrs}$. The island height also followed a power law with time of the form of Equation 3. The calculation of the power exponent comes from the fit of Equation (89) to the experimental data. As explained above, for cluster growth $t_{o}$ is the time when the cluster is first observed in the image, which might have an error due to image acquisition time of about 15 minutes. In the case of an island in a de-wetted film, such as that in Figure $11, t_{0}$ does not have the same meaning because the island already exists when it de-attaches from the film. Therefore, $t_{0}$ is chosen to be a fitting variable in the determination of the power constant $\beta$. The best fitting parameters are $t_{0}=$ -0.8 hours and $\beta=0.25$. In this case the exponent is also consistent with diffusion limited case for cluster growth. 


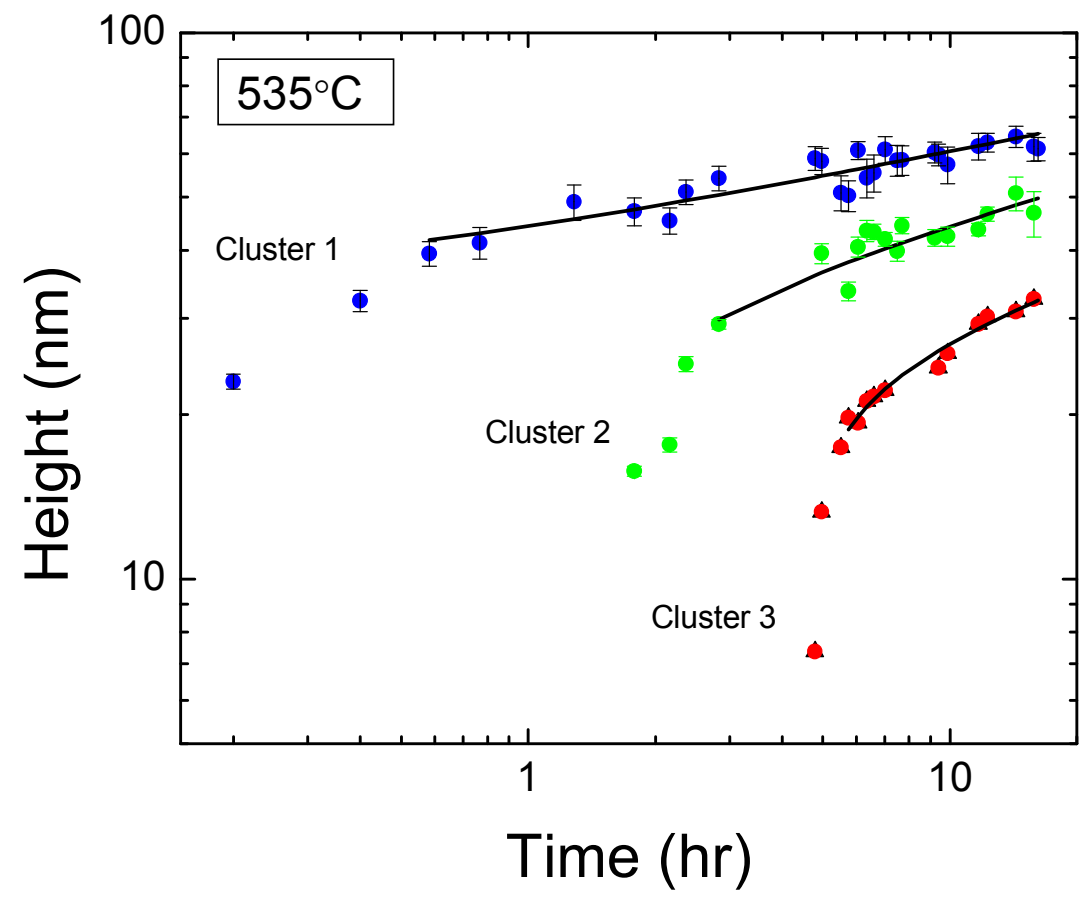

Figure 43. (a) Cluster height as a function of annealing time for a cobalt film annealed at $535^{\circ} \mathrm{C}$. The black curves depict the fit of Equation (88) to the experimental data. 


\begin{tabular}{c|ccc}
\hline Cluster & $\begin{array}{c}\mathrm{h}_{0} \\
(\mathrm{~nm})\end{array}$ & $\begin{array}{c}\mathrm{t}_{0} \\
(\mathrm{hr})\end{array}$ & $\beta$ \\
\hline 1 & $14.8 \pm 12.7$ & $-0.35 \pm 0.30$ & $0.16 \pm 0.01$ \\
2 & $38.3 \pm 10.7$ & $1.39 \pm 0.39$ & $0.22 \pm 0.01$ \\
3 & $80.0 \pm 13.4$ & $4.50 \pm 0.75$ & $0.24 \pm 0.03$ \\
\hline
\end{tabular}

Table 8. Fitting parameters for cluster height evolution for an annealing temperature of $535^{\circ} \mathrm{C}$.

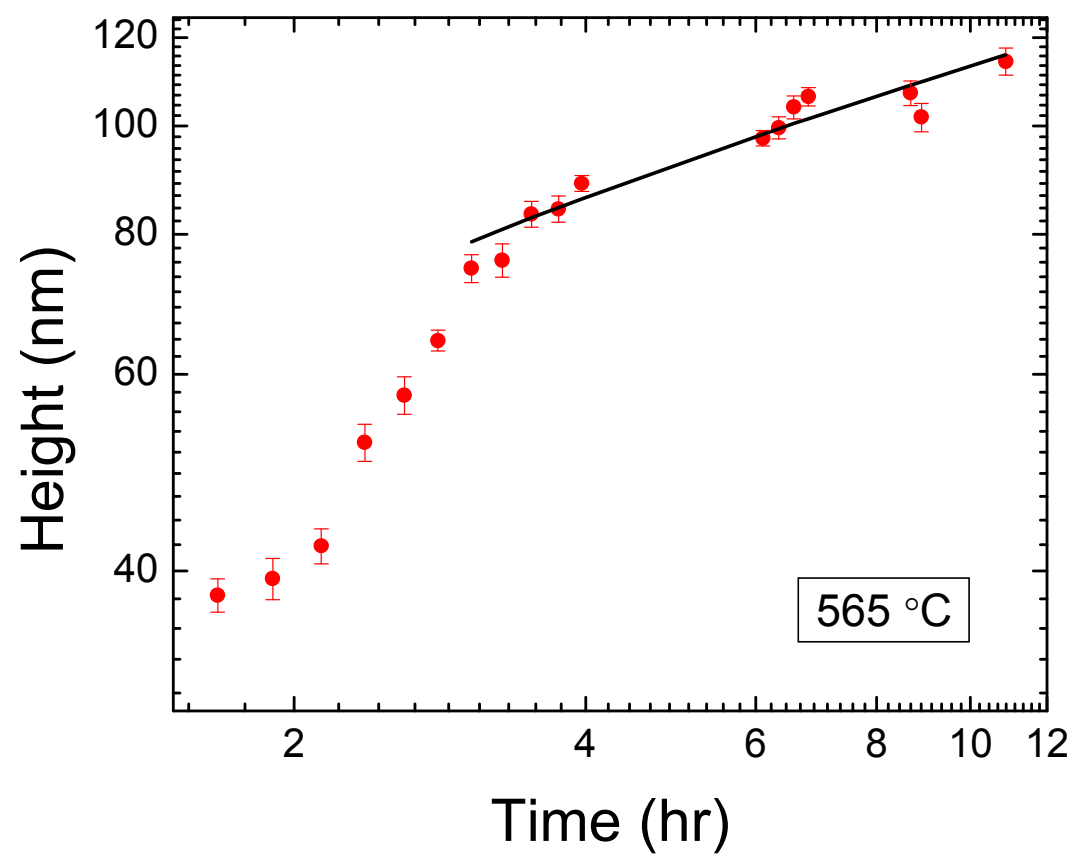

Figure 44. Cluster height as a function of annealing time for a temperature of $565{ }^{\circ} \mathrm{C}$. The black line corresponds to the fit of Equation (89) to the experimental data excluding the first 6 data points. 


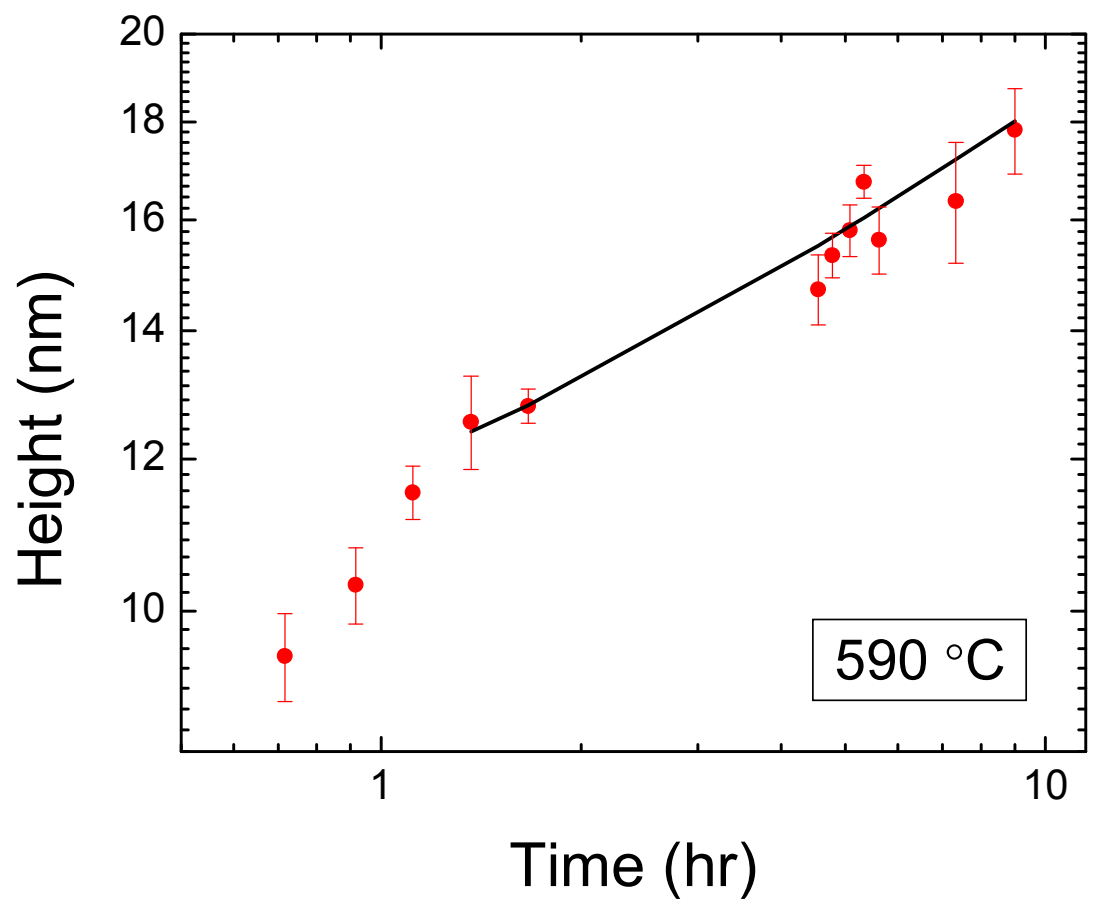

Figure 45. (a) Cluster height as a function of time for a cobalt film annealed at $590^{\circ} \mathrm{C}$. The black line is a fit to a power law excluding the first 3 data points.

\begin{tabular}{c|ccc}
\hline $\begin{array}{c}\text { Temperature } \\
{ }^{\circ} \mathrm{C}\end{array}$ & $\begin{array}{c}\mathrm{h}_{0} \\
(\mathrm{~nm})\end{array}$ & $\begin{array}{c}\mathrm{t}_{0} \\
(\mathrm{hr})\end{array}$ & $\beta$ \\
\hline 565 & $55.6 \pm 10.0$ & $0.9 \pm 1.0$ & $0.25 \pm 0.01$ \\
590 & $8.2 \pm 2.0$ & $-0.8 \pm 1.0$ & $0.25 \pm 0.02$ \\
\hline
\end{tabular}

Table 9. Fitting parameters for cluster height evolution for different annealing temperatures. 


\subsection{AREA OF CLUSTERS}

The area of the islands was measured as a function of time for the cobalt film annealed at $535{ }^{\circ} \mathrm{C}$. The area was calculated by taking a window around each cluster and computing the number of pixels inside the window above some intensity threshold. Because the threshold depends on the background intensity around the islands and this value can be influenced by small objects around the

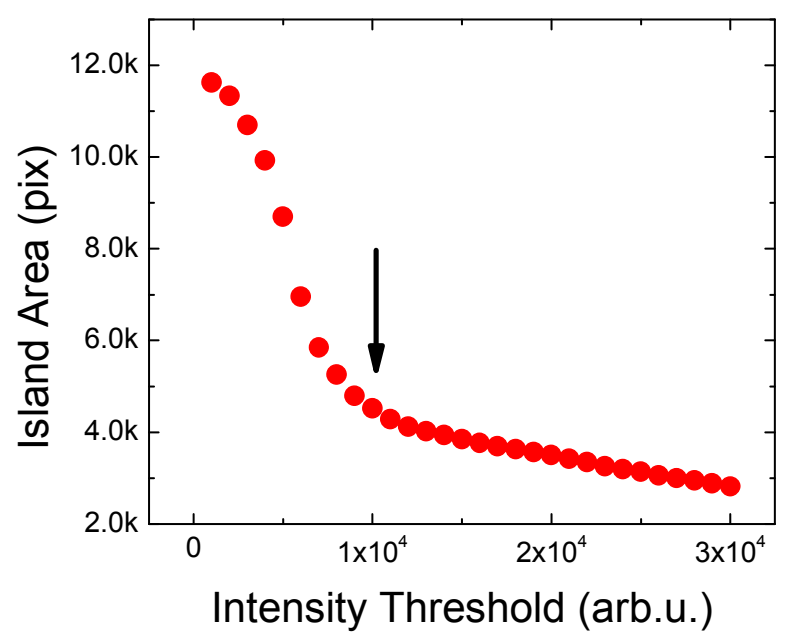

Figure 46. Island area for different thresholds. The island size was measured right in the zone when the area increases sharply as indicated by the black arrow.

large clusters, great care was taken so as to not overestimate or underestimate the island area. In order to prevent this, the area of each cluster was measured for different thresholds as depicted in Figure 46. If the threshold is too big only a few pixels will have intensities larger than the threshold and a small island area is obtained. On the other hand, if the threshold is too small, the area of the cluster will be highly overestimated. Therefore, the cluster area was calculated only in the zone where the area increased abruptly (as shown by the black arrow in 
Figure 46), which is an indication that pixels were only counted above the threshold around the island. Figure 47 shows the area evolution in time for the three clusters observed at $535{ }^{\circ} \mathrm{C}$. For all clusters, the area grew very quickly during the first hour, reaching a maximum after approximately 4-5 hrs. Afterwards, the area seemed to stay constant for both clusters. Fitting a power law to the area $A$ as a function of time of the form $A \sim\left(t / t_{0-}-1\right)^{\xi}$ for cluster $2, t>$ $4 \mathrm{hr}$ and $t_{0}=2 \mathrm{hr}$, an exponent $\xi=0.05 \pm 0.04$ is obtained and a very poor correlation $r \sim 0.49$, which indicates that the area remained almost unchanged after 4 hours of heating. This is also observed for cluster 1, although the uncertainties are larger than those for cluster 2 . This result is surprising because the clusters' height continued increasing for long annealing times, and therefore there must be a mechanism that favors the vertical growth of the islands over lateral growth in the substrate plane. 
The area of cluster 3 (see Figure 9) also increased as a function of time, but it did not show a clear tendency towards saturation as seen for clusters 1 and 2 . There might be several explanations for this. First, the determination of the area for cluster 3 is more difficult because of its smaller size and therefore it is more strongly affected by small drifts during the scan. Second, the small intensity above the threshold makes the detection of the cluster more difficult. Finally, the
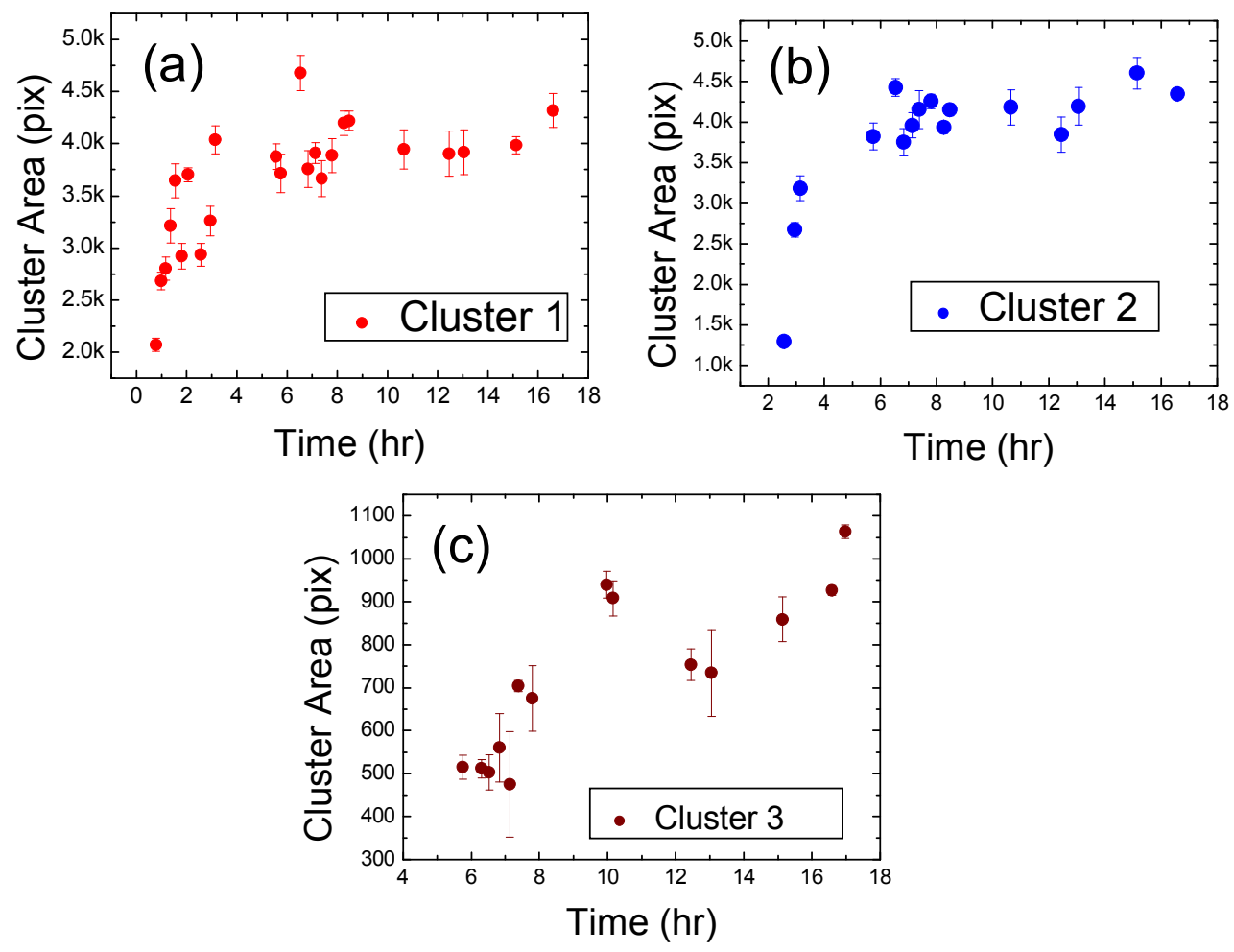

Figure 47. Area of the clusters as a function of time.

area can be distorted by other small clusters growing in the neighborhood. The procedure to compute the island's area works well if the threshold is well defined around each object as it happen for the clusters selected at $535^{\circ} \mathrm{C}$; however it fails for the clusters imaged at $565{ }^{\circ} \mathrm{C}$ because the islands grow very close to each other, making it impossible to determine the threshold. 
Another approach used to analyze the area as a function of time is to take line profiles of the islands at two different times for a fixed annealing temperature. Figure 48 shows line profiles for annealing temperatures of $535^{\circ} \mathrm{C}, 565{ }^{\circ} \mathrm{C}$ and $590{ }^{\circ} \mathrm{C}$ in the direction parallel to the scan direction ( $x$-axis) and perpendicular to the scan direction ( $y$-axis). At $535{ }^{\circ} \mathrm{C}$ the profiles for a cluster at $2.4 \mathrm{hr}$ (blue dots) and at $15.8 \mathrm{hr}$ (red dots) are shown. Despite the fact that these two measurements were separated by a long period of time, the basal area of the cluster grew only $7 \%$, which is small when compared with he increase in height of about $70 \%$. This behavior is true for both axes. Also, the basic shape of the cluster (flat top and steep edges) is almost unchanged with time. At $565{ }^{\circ} \mathrm{C}$ and $590{ }^{\circ} \mathrm{C}$ the same behavior was observed, where the basal area of the clusters was almost unchanged and the island growth occurred perpendicular to the film plane only. The clusters maintained their shapes and the only difference between short and long annealing times seems to be a proportionality factor along the z-axis. This is indicative of self-similarity i.e. statistically speaking, once the clusters nucleate, they look the same regardless of the annealing time. Therefore, if the same morphology of a single cluster is observed at 2 different annealing times, the morphology along the z-axis should just differ by a proportionality factor. The line profiles along the $x$ - and $y$-axes confirm the observation of the cluster's area at $535^{\circ} \mathrm{C}$ in the sense that most of the variation of the basal area should happen during the first 2 or 3 hours of annealing, after which the clusters evolve perpendicular to the film surface only. 

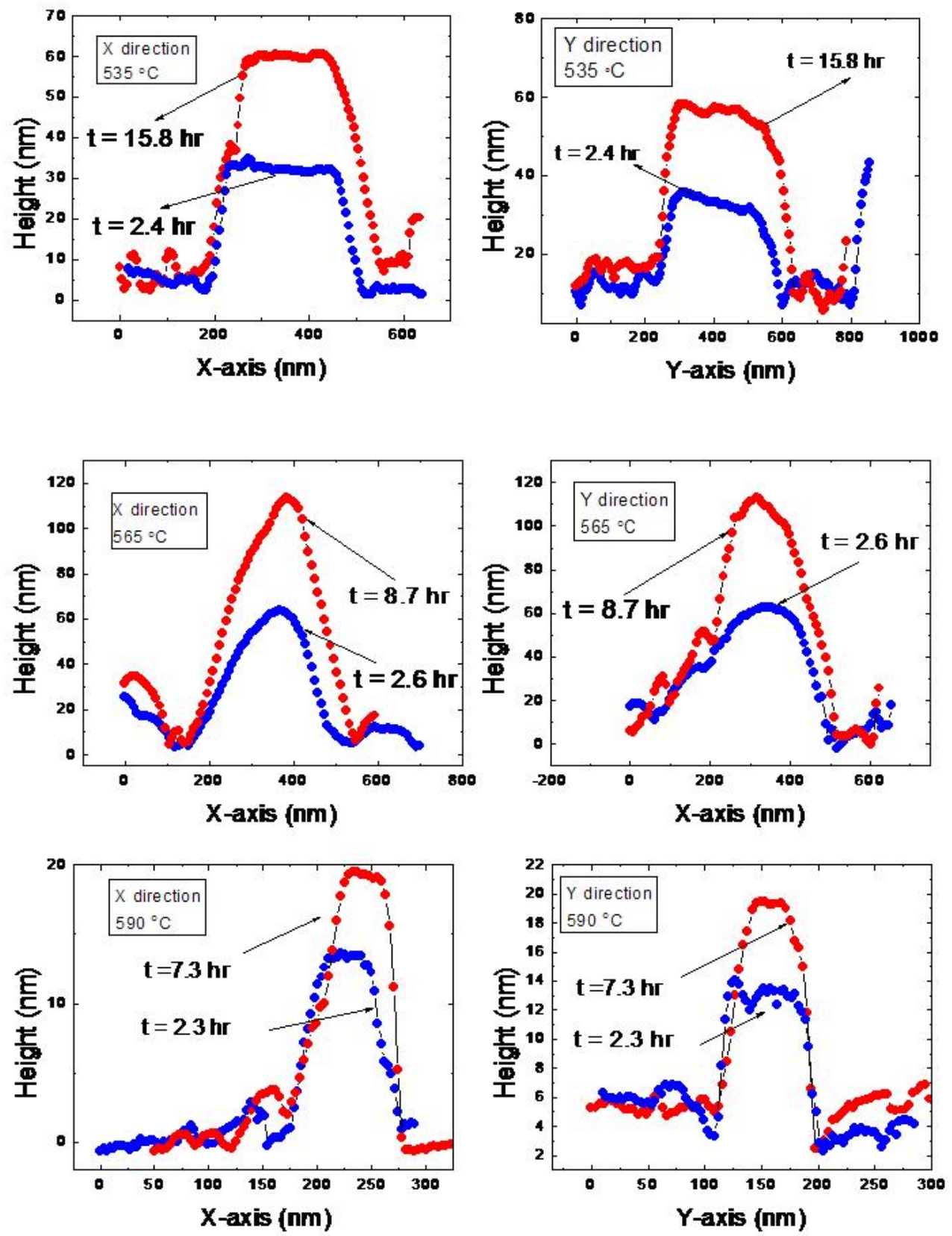

Figure 48. Line profiles for 3 different annealing temperatures of $\mathbf{5 3 5}$ ${ }^{\circ} \mathrm{C}, 565{ }^{\circ} \mathrm{C}$ and $590{ }^{\circ} \mathrm{C}$. The left hand side graphs show the line profiles along the $X$-axis of the clusters and the right hand side shows the profile along the $Y$-axis. 


\subsection{POWER SPECTRUM}

Another method to obtain useful information from the AFM images is to convert them into the reciprocal space through a Fourier transform. The Fourier transform of a 2 dimensional discrete image can be obtained by the equation: ${ }^{59}$

$$
F\left(q_{x}, q_{y}\right)=\frac{1}{M N} \sum_{i=0}^{M-1} \sum_{j=0}^{N-1} f(i, j) \times \exp \left\{-i 2 \pi\left(\frac{q_{x} i}{M}+\frac{q_{y} j}{N}\right)\right\},
$$

where $M$ and $N$ are the size of the image in pixels, $f(i, j)$ is the intensity of the image at the position $(i, j), q_{x}$ is the wavevector in the direction of the scanning and $q_{y}$ is the wavevector in the direction perpendicular to the scan. This formula might seem straightforward, but in reality it requires long computational times for even small matrices. In practice a numerical method called fast Fourier transform (FFT) is normally used because it reduces the number of computations of $P$ pixels from a factor of $2 P^{2}$ to $2 P \ln (P)$. The FFT code of Origin Lab software was used for this purpose.

The power spectrum was computed for the AFM images at different

temperatures and annealing times as: $\left|F\left(q_{x}, a_{y}\right)\right|^{2}$. The power spectrum is a powerful tool to observe the coherence of the clusters in the film. For instance if there is any characteristic separation between clusters and this separation is isotropic, the $2 \mathrm{D}$ power spectrum should have a central rim, much like the one shown in Figure 17. The radius of this rim $q_{r}$ (with $q_{r}{ }^{2}=q_{x}{ }^{2}+q_{y}{ }^{2}$ ) is equal to $2 \pi \lambda^{-1}$, where $\lambda$ is the characteristic separation of the clusters in real space. The value of this parameter depends the mechanism of cluster formation as seen in the previous chapters. The power spectra for 3 different samples annealed at $535{ }^{\circ} \mathrm{C}, 565{ }^{\circ} \mathrm{C}$ and $590{ }^{\circ} \mathrm{C}$ are shown in Figure 49 and Figure 50. The power spectra center (i.e. $q_{x}=q_{y}=0$ ) is located in the central point of the images; white regions represent high intensity locations and grey or black represent low intensity locations. The plot on top of the $2 \mathrm{D}$ power spectra depicts the profile 
along yellow line. The main characteristic of the power spectra of all 3 temperatures is a bright circular spot in the center that rapidly decays for large radii. The circular shape of the bright spot is an indication of the incoherence of the cluster positions in the AFM images i.e., the distribution of the islands is random. This random distribution of islands means that there is no characteristic separation between them. Also the power spectra are almost circular, which means that the islands distribution is isotropic in $2 \mathrm{D}$ space. There is no significant difference in the power spectra obtained for different temperatures or annealing times. Figure 51 and Figure 52 show the radial intensity of the power spectra for different annealing times. The plots show a smooth decay of the power spectrum with $q$ and no indication of a preferential wavelength as seen in the $2 \mathrm{D}$ spectrum. Because the AFM image for the $565^{\circ} \mathrm{C}$ sample clearly shows a separation of the islands between 200-300 nm (Figure 32), a well-defined island periodicity should have resulted in a peak in the spectrum at $q \sim 0.02-0.03$ $\mathrm{nm}^{-1}$, but nothing is visible in Figure 18 except for the shortest time (1.7 hrs). This result does not necessarily mean that the annealed films lacked any coherence because the power spectrum is influenced by the image size, but it does mean that there is no coherence for wavelengths spanning the image size $(2 \mu \mathrm{m})$ or smaller than the pixel size or the size of tip $(\sim 5 \mathrm{~nm})$, whichever is larger. 

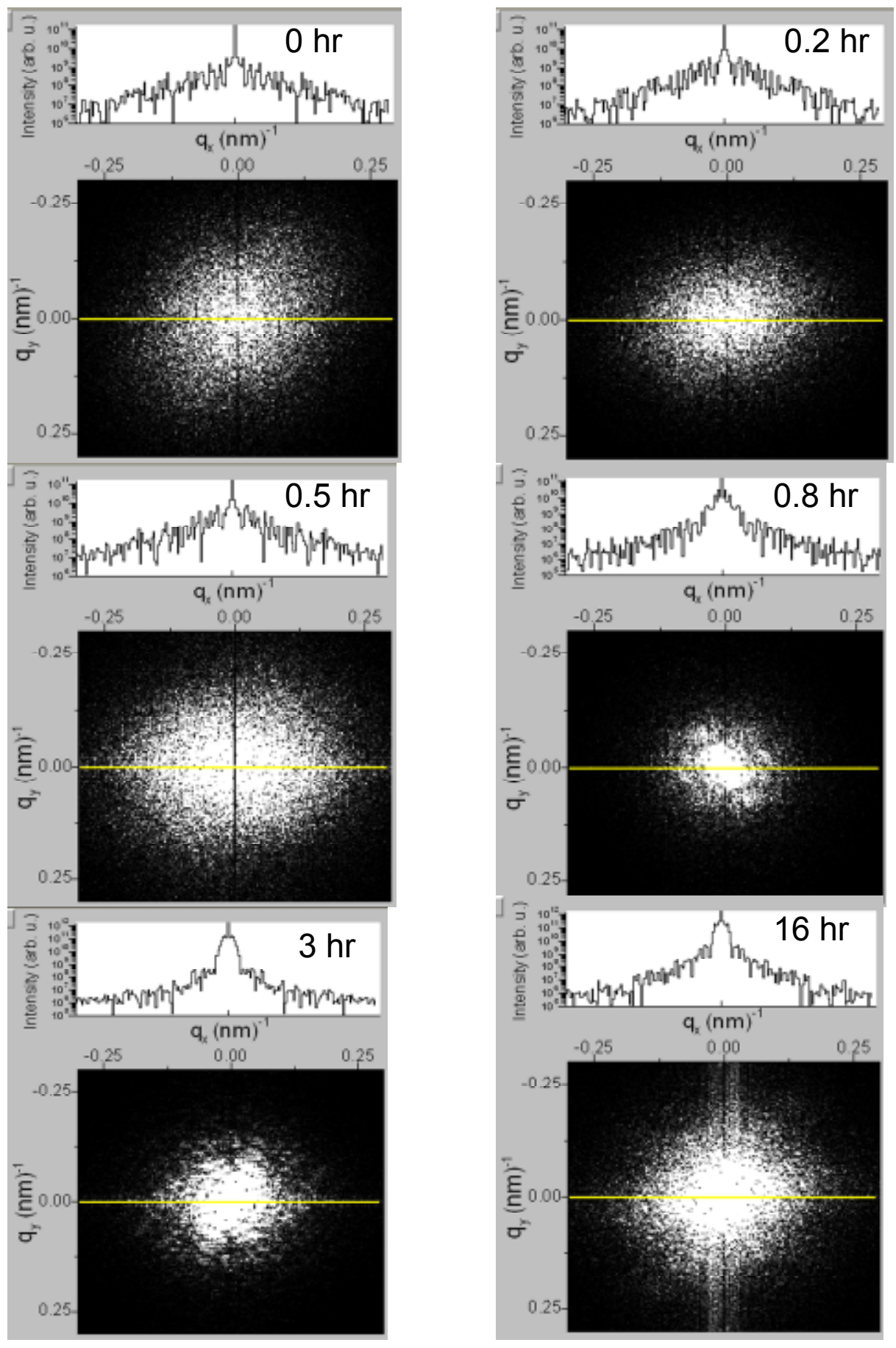

Figure 49. Power spectrum of a cobalt film at $535^{\circ} \mathrm{C}$ for different annealing times. 

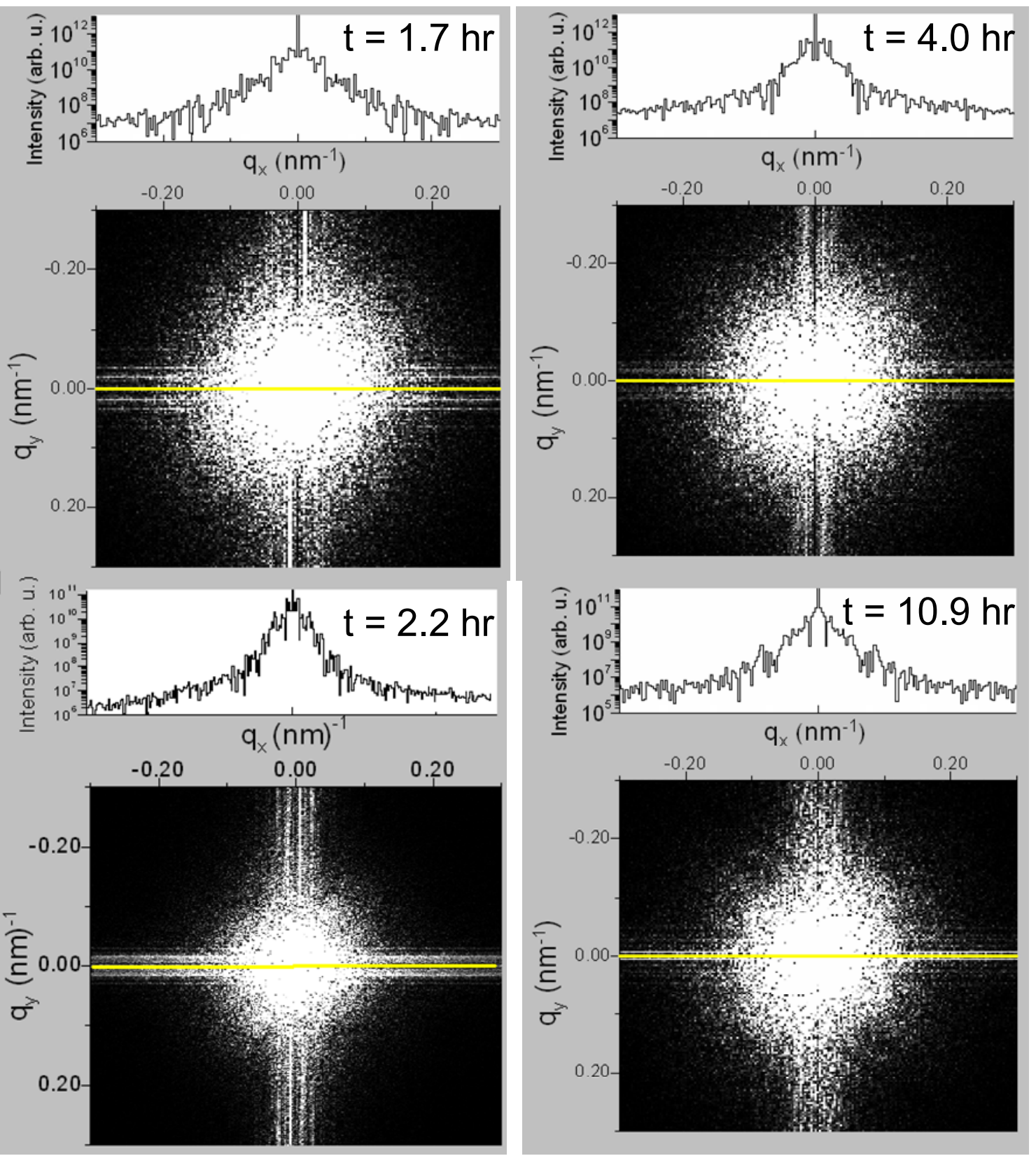

Figure 50. Power spectrum of a cobalt film at $565^{\circ} \mathrm{C}$ for different annealing times. 


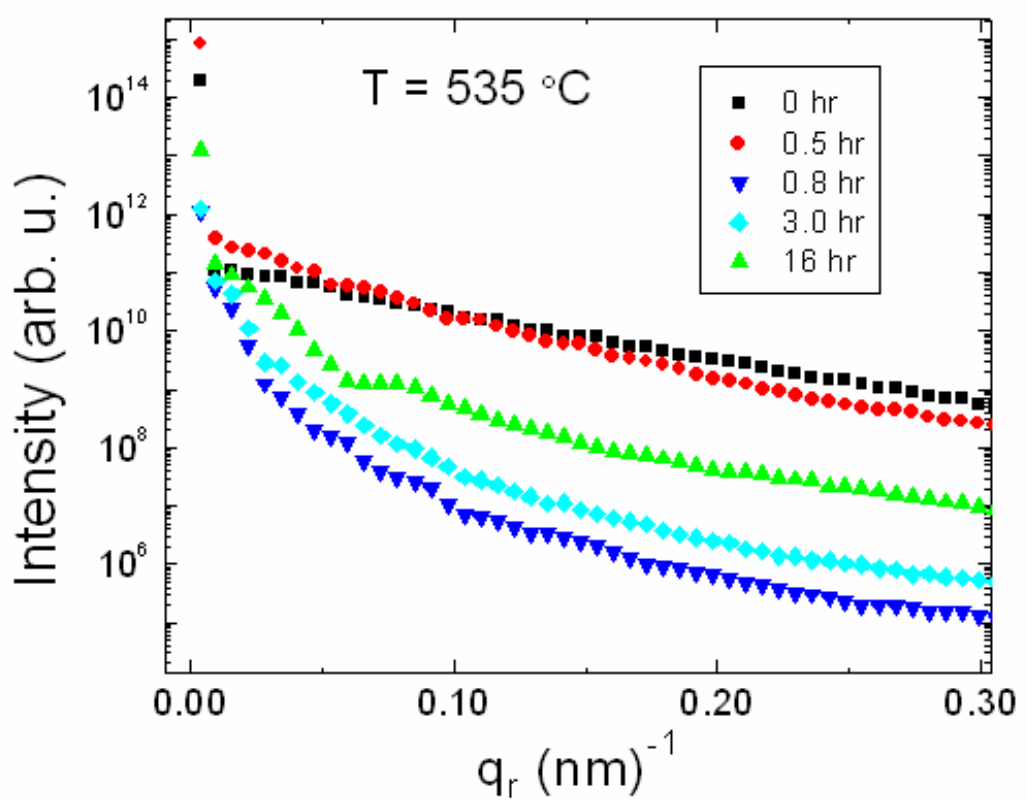

Figure 51. Radial average of the power spectrum for the cobalt film annealed at $535^{\circ} \mathrm{C}$.

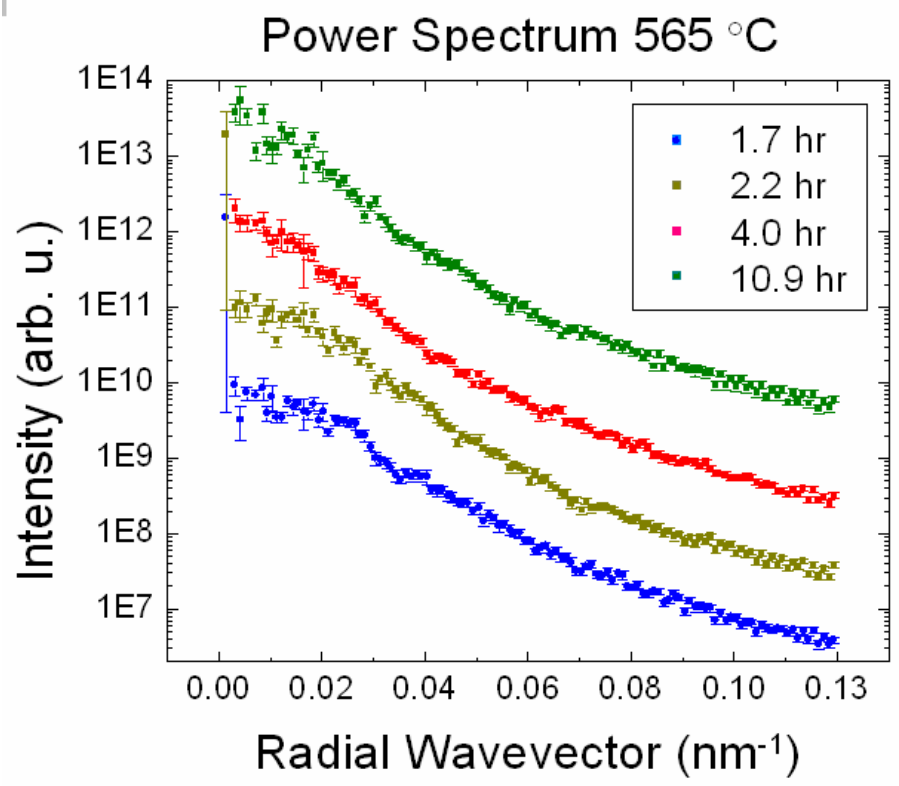

Figure 52. Radial average of the power spectrum for the cobalt film annealed at $565^{\circ} \mathrm{C}$. 


\subsection{AUTOCORRELATION}

The autocorrelation is another powerful tool to statistically examine features in the images such as cluster diameter and cluster separation. For a one dimensional continuous function $f(x)$ the autocorrelation is defined as

$$
f * f=\int_{-\infty}^{\infty} f(u) f(x-u) d u .
$$

An easy way to calculate the autocorrelation function is through the autocorrelation theorem which says that if $F(q)$ is the Fourier Transform of some function $f(x)$, then the autocorrelation of that function is the inverse of the Fourier Transform of its power spectrum $F^{2}(q)$, or in another words, ${ }^{59}$

$$
\begin{aligned}
& f(x, y) \rightarrow F\left(q_{x}, q_{y}\right) \\
& f * f \rightarrow\left|F^{2}\left(q_{x}, q_{y}\right)\right|
\end{aligned}
$$

The autocorrelation was computed by inverting the power spectrum obtained in the previous section for the AFM images using the Origin Lab software. 

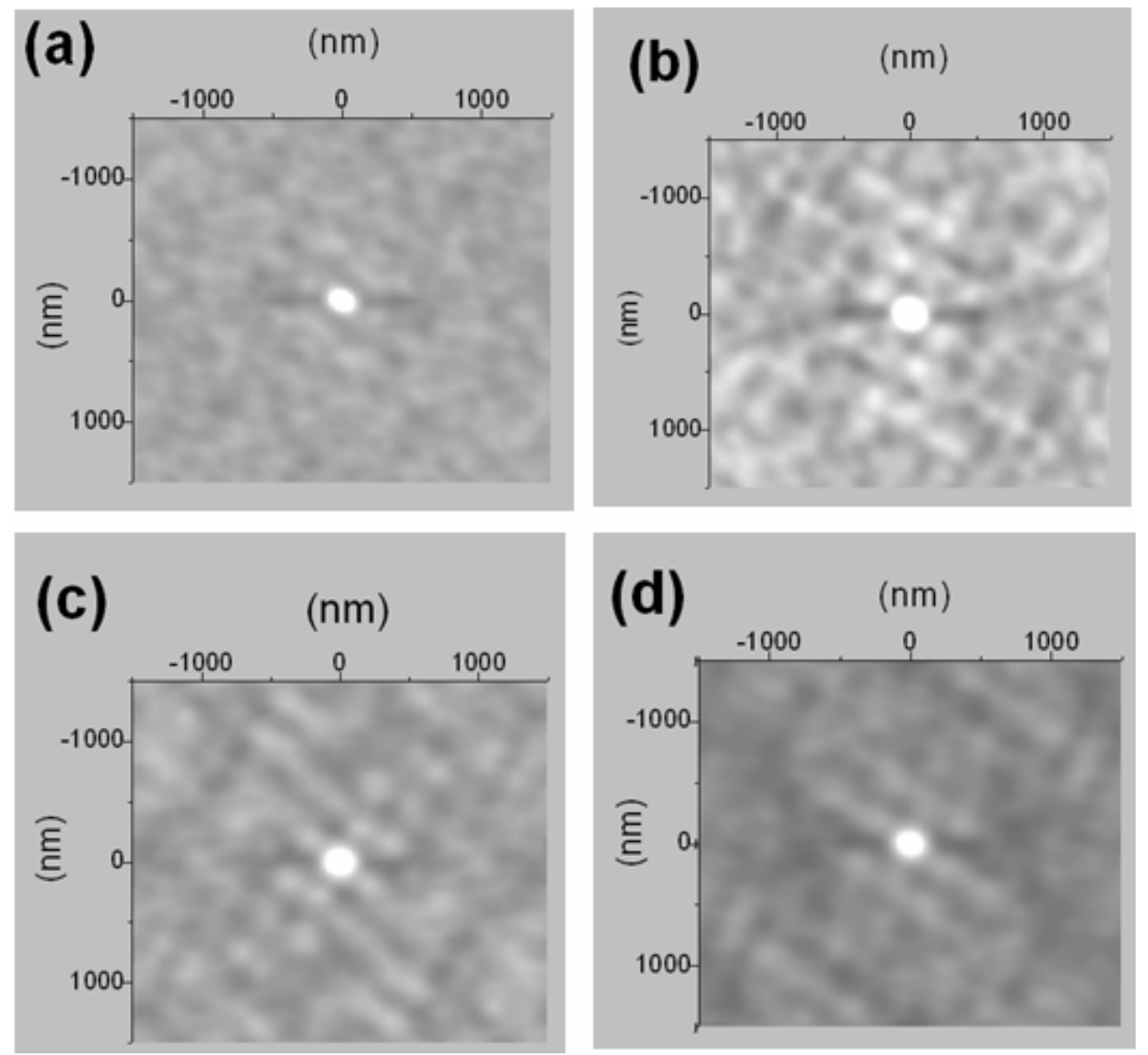

Figure 53. Autocorrelation function for the cobalt film annealed at $565{ }^{\circ} \mathrm{C}$. (a) after $1.7 \mathrm{hr}$ of annealing, (b) $2.4 \mathrm{hr}$, (c) $8.9 \mathrm{hr}$ and (d) $10.8 \mathrm{hr}$. White and light gray are places of high intensity and dark gray are places of low intensity.

The height-height correlation function in 2D can be seen in Figure 53 for the images taken at $565{ }^{\circ} \mathrm{C}$. The main feature of the autocorrelation function is a very intense central peak. The width of this peak is proportional to the average width of the clusters, depicted in Figure 54 as $w$. In order to compute $w$, the radial average of the height-height correlation function was calculated for $r^{2}=x^{2}+y^{2}$, where $\mathrm{x}$ and $\mathrm{y}$ are the coordinates of the pixels in each autocorrelation frame. The results are shown in Figure 55. The width w was 
computed as the full width at half maximum (FWHM) of the peak centered at $r=$ 0 which is supposed to have a Gaussian shape. A secondary peak can be observed in the height-height correlation function as shown in Figure 55; the distance between the central peak and the secondary peak corresponds to the average intercluster separation $\lambda$, or in other words, it represents the average distance between one peak and the nearest neighbor. These two parameters were calculated for the film annealed at $565{ }^{\circ} \mathrm{C}$ for different annealing times, as depicted in Figure 57. The average width $w$ (red dots) changed only in the first 2 hours of annealing, from $100 \mathrm{~nm}$ to $200 \mathrm{~nm}$, but after that it remained unchanged. This behavior was seen by measuring the area of single clusters at $565{ }^{\circ} \mathrm{C}$, but in this is a statistical proof of that behavior. The intercluster separation $\lambda$ (green dots) changed only during the first 2 hours and after that it did not vary significantly with time. This experiment indicates that the average intercluster separation at early times ranged between $200-300 \mathrm{~nm}$ and, as was explained in the introduction, this parameter is important in determining the interaction responsible for the formation of the clusters.

For $535{ }^{\circ} \mathrm{C}$ the average cluster width $w$ and the average intercluster separation $\lambda$ were also measured as a function of time in the same way. The results can be seen in Figure 56. Before 0.8 hours the cluster width was less than $100 \mathrm{~nm}$ while the intercluster separation ranged between $150 \mathrm{~nm}$ and $200 \mathrm{~nm}$. For annealing times longer than 0.8 hours, the values of $w$ and $\lambda$ increased drastically, but this increase has a poor statistical significance because it is due to the formation of the first large cluster (cluster 1) seen in Figure 40. In any case, it is possible to conclude that the annealing temperature does not affect greatly the intercluster separation, at least in the temperature range of $535{ }^{\circ} \mathrm{C}$ to $565{ }^{\circ} \mathrm{C}$, and that the intercluster separation fluctuates between $150 \mathrm{~nm}$ and $300 \mathrm{~nm}$. 


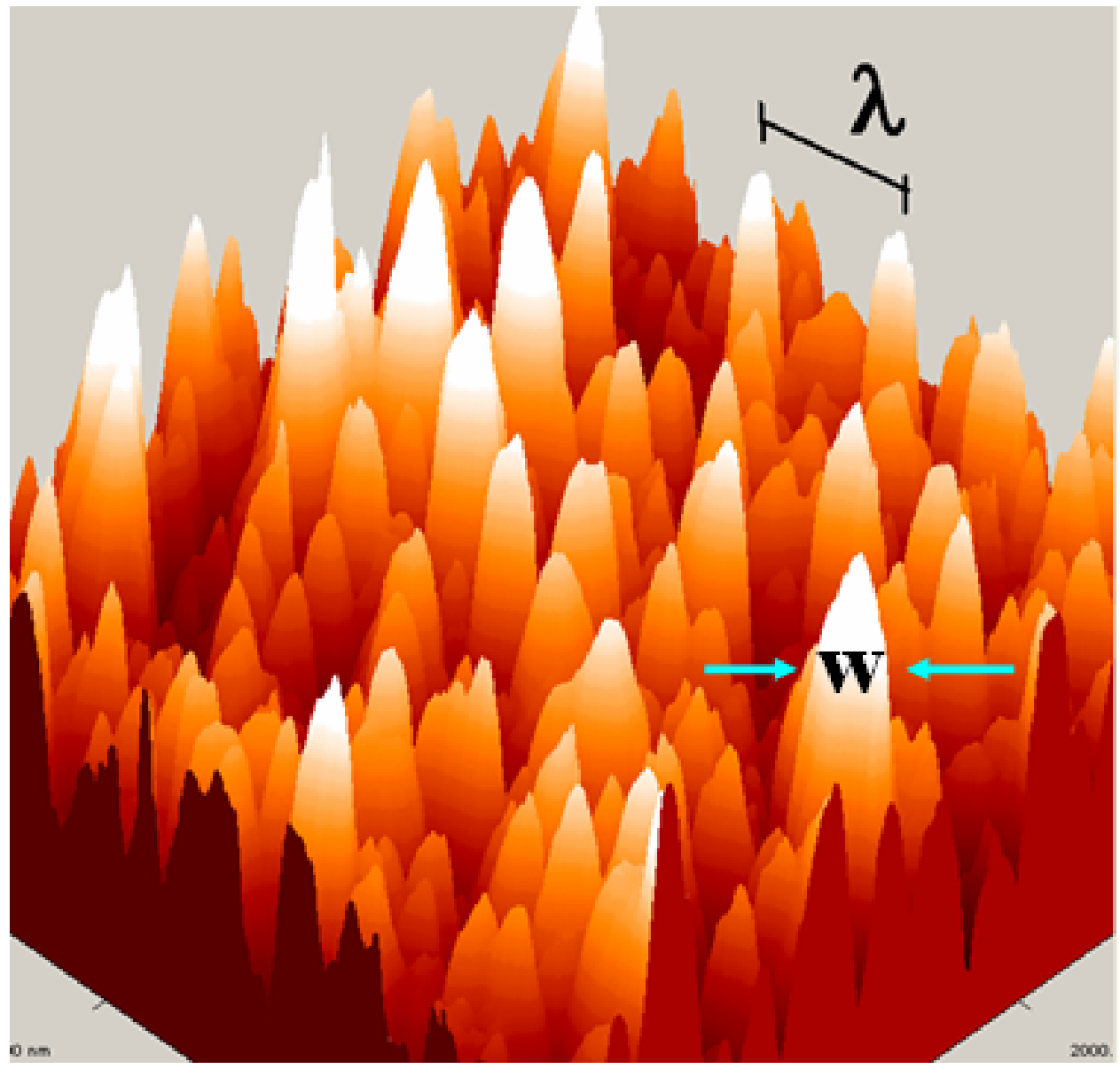

Figure 54. AFM image of a cobalt film annealed at $565{ }^{\circ} \mathrm{C}$ depicting the cluster width $w$ and the intercluster separation $\lambda$. 

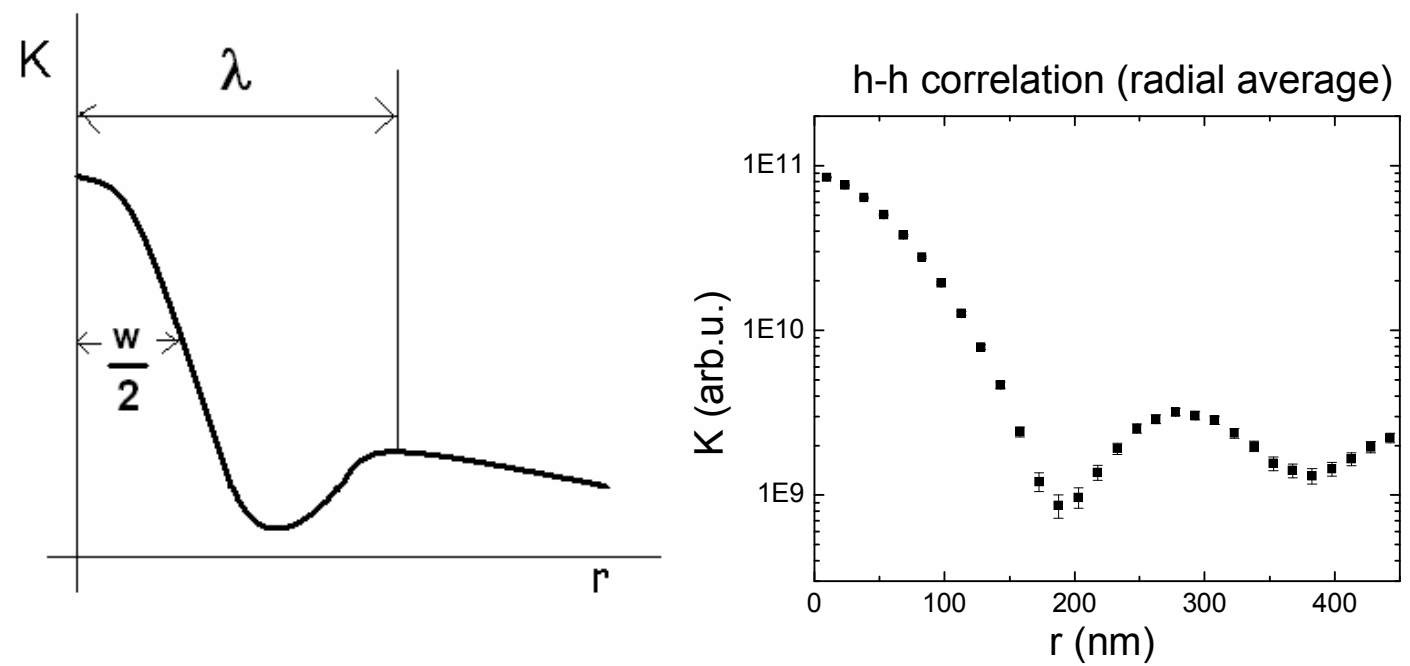

Figure 55. Radial average of the height-height correlation function. Left: sketch of the radial average with the two main parameters $w$ and $\lambda$ indicated in the figure. Right: experimental radial average of the heightheight correlation function for a cobalt film annealed at $565^{\circ} \mathrm{C}$ for $1.7 \mathrm{hr}$.

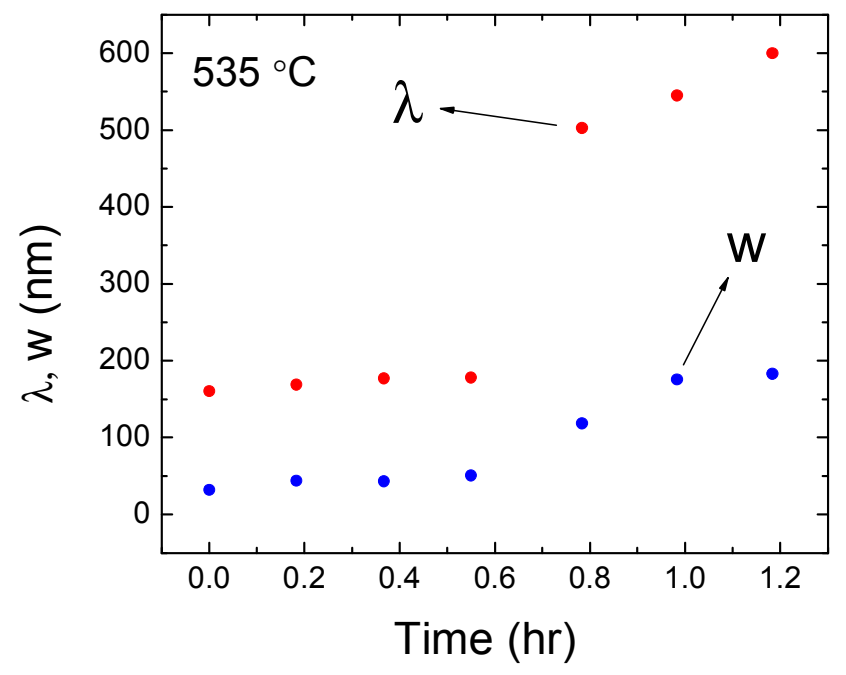

Figure 56. Average intercluster separation and cluster width for a cobalt thin film annealed at $535^{\circ} \mathrm{C}$. 


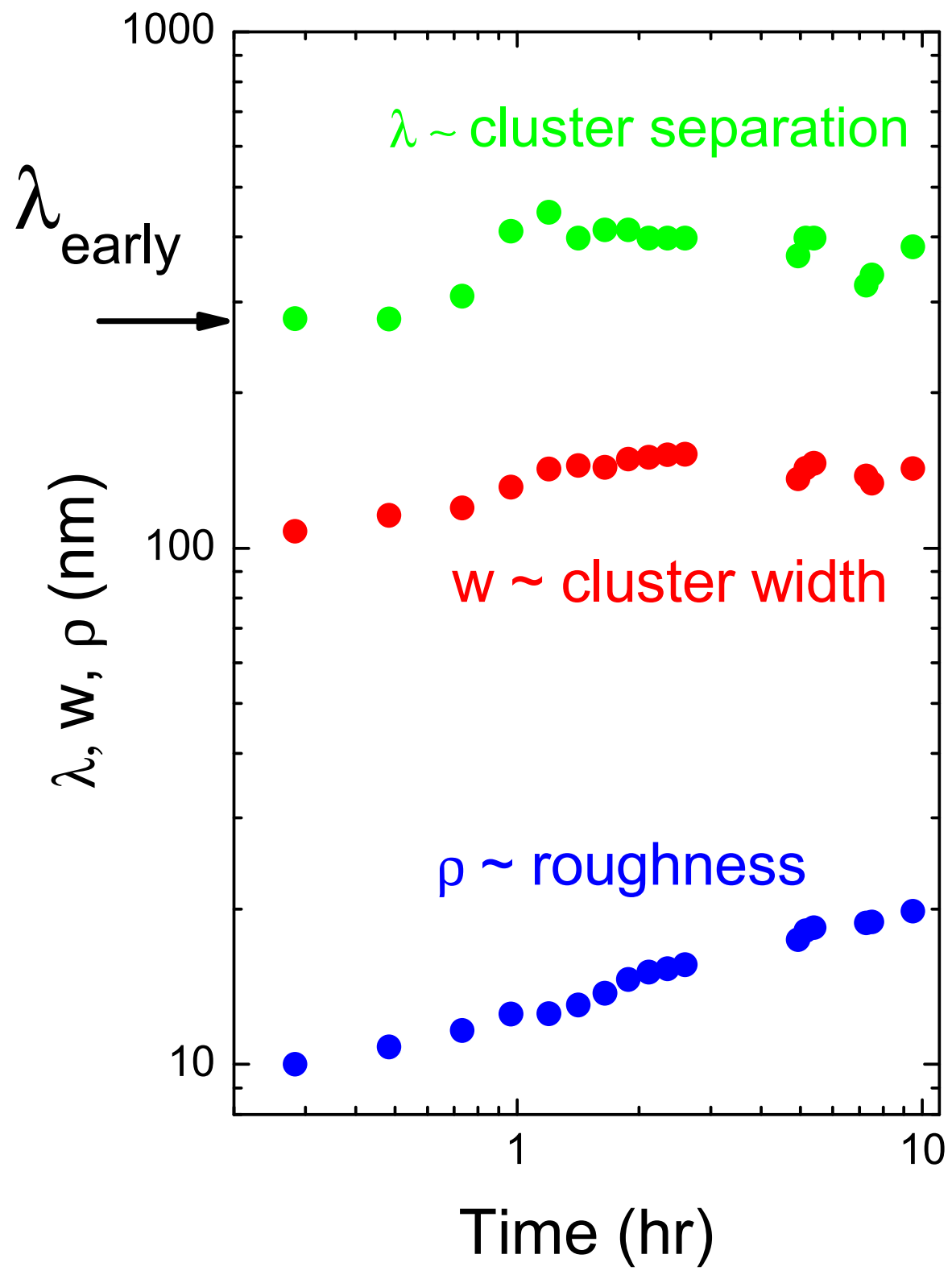

Figure 57. Cluster width $w$, intercluster separation $\lambda$ and image roughness $\rho$ as a function of time for the cobalt film annealed at $565{ }^{\circ} \mathrm{C}$. 


\section{DISCUSION}

This section discusses the main results found for the de-wetting experiments on cobalt thin films at different temperatures.

In Figure 58 it is possible to observe the summary of the different variables studied at early and late annealing times. The term late refers to annealing times longer than 2 hours. The height of individual clusters was followed as a function of time. The clusters' height increased with time in two different ways. For short annealing times the growth was exponential, which is consistent with surface instabilities ${ }^{5}$ of the surface dynamics. These instabilities are due the interplay of the Mullins' term for surface diffusion, which tends to smooth out surfaces and

$$
\mathrm{T}=535^{\circ} \mathrm{C} \quad \mathrm{T}=565^{\circ} \mathrm{C} \quad \mathrm{T}=590^{\circ} \mathrm{C}
$$

$\rho$ vs $t$

$$
\rho \sim \mathrm{t}^{\alpha} \text { (late) } \quad \rho \sim \mathrm{t}^{\alpha} \text { (late) } \quad \rho \sim \mathrm{t}^{\alpha} \text { (late) }
$$

$$
\alpha \sim 0.20
$$

$\alpha \sim 0.21$

$\alpha \sim 0.16$

\begin{tabular}{lccc}
\hline H vs t & $\begin{array}{c}\text { Exponential (early) } \\
\mathrm{H} \sim \mathrm{t}^{\alpha}\end{array}$ & Exponential (early) & Exponential (early) \\
& $\alpha \sim 0.16-0.24$ (late) & $\begin{array}{c}\mathrm{H} \sim \mathrm{t}^{\alpha} \\
\alpha \sim 0.25 \text { (late) }\end{array}$ & $\begin{array}{c}\mathrm{H}^{\alpha} \mathrm{t}^{\alpha} \\
\alpha \sim 0.25 \text { (late) }\end{array}$ \\
\hline A vs t & Constant (late) & Constant (late) & Constant (late) \\
\hline W vs t & ?? (late) & Constant (late) & $? ?$ \\
& & & $?$ \\
A vs t & $150-200 \mathrm{~nm}$ (early) & 200-300 nm (early) & $? ?$
\end{tabular}

Figure 58. Overview of the main results found on cobalt de-wetting 
the Casimir interaction across the film. Surface instabilities are predicted any time that $\phi^{\prime \prime}(\mathrm{h})<0$, and therefore the nature of the interaction cannot be determined by analyzing the cluster's growth. Surface instabilities leading to dewetting have been observed on thin films of polymers ${ }^{3}$ but the experiments have been carried out on films quenched after annealing. To the best of my knowledge, there are no experiments of this kind carried out in real time upon annealing. On the other hand, experiments of de-wetting of solids focus on finding interfacial energies or late evolution of metal clusters due to ripening processes and therefore it is believed that this is the first time that surface

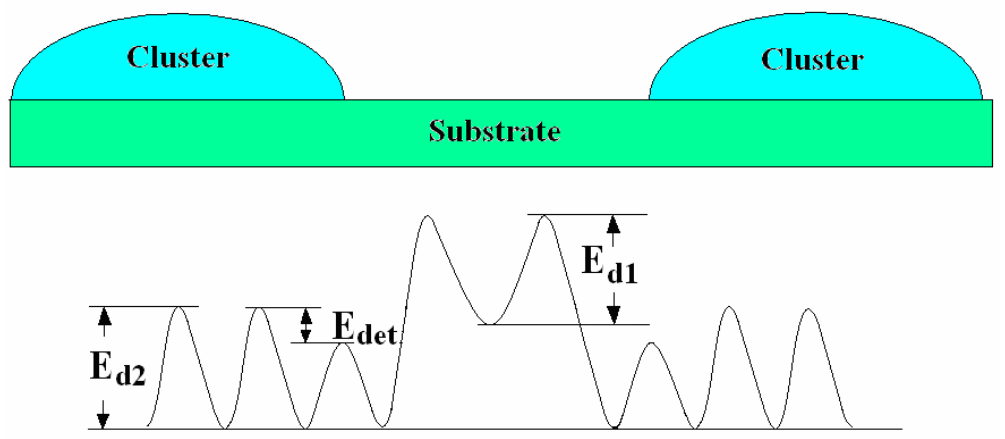

Figure 59 Surface barrier energies for two neighbor islands. $E_{\text {det }}$ is the attachment/detachment energy, $E_{d 1}$ is the diffusion between islands and $E_{d 2}$ is the diffusion energy on top of the clusters.

instabilities have been observed in-situ for a solid de-wetting process. For late annealing times, height increases as a function of time with a power law exponent close to 0.25. This exponent agrees with the diffusion limited mechanism for $3 \mathrm{D} / 2 \mathrm{D}$ systems ${ }^{17}$ (3 dimensional clusters with diffusion in 2 dimensions) seen also in magnesium silicide islands on $\mathrm{Si}(001){ }^{12}$ This mechanism was described theoretically in a previous section but it is instructive to revise it in a phenomenological way as shown in Figure 59. 
The energy $E_{d 1}$ is the diffusion energy for an adatom located in between the islands. $E_{d 2}$ is the diffusion energy for an adatom located on the cluster's surface and $E_{\text {det }}$ is the energy barrier for attachment or detachment of an adatom from the cluster's edge. The magnitude of this barrier is not known but qualitatively $E_{\text {det }}<E_{d 1}$ and $E_{\text {det }}<E_{d 2}$ because only the diffusion barriers limit the adatom flux. $E_{d 1}$ should be larger than $E_{d 2}$ because adatoms diffusing in areas between the clusters might randomly come closer to an island and to end up trapped inside of it. On the other hand, an adatom diffusing on the surface of an island might have the energy to detach from the island edge but might not have enough energy to overcome the larger diffusion energy $E_{\mathrm{d} 2}$. Hence, is more likely that adatoms detached from an island will re-attach again to it, thus resulting in a net adatom flux towards the island and leading to the cluster's growth. The roughness of the cobalt films is another useful parameter. Films annealed at higher temperatures suffered a faster roughening process and at late times the roughness increased as a function of time with a power exponent of $0.16-0.21$. The smallest exponent of 0.16 is founded for the film annealed at the highest temperature of $590^{\circ} \mathrm{C}$ and might be due to saturation of the clusters' height not seen at smaller temperatures, where the roughening process is slower. On the other hand, the exponent at lower temperatures is approximately 0.20 , slightly lower than the exponent reported for the height of single clusters (0.25). This smaller value might come about from the fact that cluster growth is not simultaneous (i.e. some clusters start growing earlier than others as seen at $535{ }^{\circ} \mathrm{C}$ ), or from an increasing average image height $\langle H\rangle$ (as was observed in the annealed films), which might decrease the computed roughness and consequently decrease the power exponent over time.

It was mentioned before that the clusters' height increased with time with a power law of 0.25 consistent with 3D/2D ripening. However, one unusual behavior of the cluster formation is that the area calculated for single clusters at different temperatures and the average cluster width obtained from the autocorrelation function remain almost constant after 2 hours of annealing. This issue goes 
against the ripening mechanism behind the 0.25 power law because in general the ripening process is isotropic, and therefore any coordinate in the clusters' size (radius or height) should in principle grow with the same power law. In the case of the Mg silicide islands on Si (001), the height increases with a power law 0.25 but as it can be seen through the images taken at different annealing times (see Figure 10) the islands' diameter also increases, which is consistent with ripening. In the experiments with cobalt, the basal area of the clusters remains almost unchanged which raises questions such as why do the clusters' area stop growing after 2 hours of annealing? And is another phenomenon that could cause this anisotropic growth?

On the other hand, it is necessary to analyze the intercluster separation. The only film with an appropriate number of clusters to perform good statistical analysis of the intercluster separation at early and late annealing times was the cobalt film annealed at $565{ }^{\circ} \mathrm{C}$. The film annealed at $535{ }^{\circ} \mathrm{C}$ showed good statistics only for short annealing times, but for longer ones it was affected by the formation of two large clusters in the image. The film annealed at $590{ }^{\circ} \mathrm{C}$ likely underwent corrugations at a very early time, when the system still was not in thermal equilibrium (hence it was not stable), and therefore it was not possible to capture images at that stage. It was found that the intercluster separation at early annealing times for the cobalt films was $150 \mathrm{~nm}$ to $300 \mathrm{~nm}$ in the range of annealing temperatures of $535^{\circ} \mathrm{C}$ to $565{ }^{\circ} \mathrm{C}$. For anneals longer than 2 hours, the intercluster separation remained almost unchanged at $565{ }^{\circ} \mathrm{C}$. Table 2 shows that the calculated intercluster separation at early times for a $4 \mathrm{~nm}$ thick Co film under the influence of a Van der Waals interaction. Evidently the theoretical calculation gives a separation of $\sim 1 \mu \mathrm{m}$, which is almost one order of magnitude bigger than the experimental value for Co. In order to obtain separations of the order of $200 \mathrm{~nm}$ to $300 \mathrm{~nm}$ using a Van der Waals interaction, a Hamaker constant of $200 \times 10^{-20} \mathrm{~J}$ is required, which is one order of magnitude larger than the usual accepted value for metals. On the other hand, using the fermionic Casimir interaction described in a previous section, the intercluster 
separation takes the form: $\lambda_{\text {early }} \cong 2 \pi h_{0} \sqrt{h_{0} / a}$ where $h_{0}$ is the initial thickness of the cobalt film $(4 \mathrm{~nm})$ and $a$ the lattice parameter $(0.25 \mathrm{~nm}$ for hcp cobalt). Inserting these values on the last equation we obtain $\lambda_{\text {early }} \sim 100 \mathrm{~nm}$. This value compares favorably with the experimental separation found for annealed cobalt films. Therefore the fermionic Casimir interaction is a good candidate for a mechanism responsible of surface instabilities on cobalt thin films. In general, it is expected that this interaction is be more important for the de-wetting of metal thin films than the Van der Waals interaction, which is more suitable for insulator materials. In the future, this could be verified by growing other metallic thin films and verifying that they have smaller intercluster separations. Notice that the larger intercluster separations have been observed in insulating polymer films, as described above. It will also be important to measure the intercluster separation for cobalt thin films at early annealing times for different film thicknesses, below 5 $\mathrm{nm}$, which would lead to the determination of $\phi^{\prime \prime}(\mathrm{h})$ and the exact dependence of the Casimir interaction with the film thickness.

\section{CONCLUSIONS}

Cobalt thin films approximately $4 \mathrm{~nm}$ thick were deposited on sapphire (110) and then annealed at three different and fixed temperatures $\left(535^{\circ} \mathrm{C}, 565^{\circ} \mathrm{C}\right.$ and 590 ${ }^{\circ} \mathrm{C}$ ). Their surface dynamics was measured in-situ using a variable temperature AFM. The as-grown samples were very smooth but underwent a sudden roughening transition above $500{ }^{\circ} \mathrm{C}$. The roughening process was faster for higher annealing temperatures and had a power-law dependence on time. Individual clusters grew in two different regimes independent of the annealing temperature. For the initial stages of the annealing process, the height increased exponentially with time which is consistent with surface instabilities. For later annealing times the clusters' height grew slower with a power-law dependence of $h \sim t^{\beta}$ with $\beta \sim 0.25$, which in principle is consistent with diffusion limited process of ripening in $3 \mathrm{D} / 2 \mathrm{D}$ systems. On the other hand, the basal area of the clusters 
remained almost unchanged for most of the annealing process, which disagrees with ripening models. An statistical study of the intercluster separation at $535{ }^{\circ} \mathrm{C}$ and $565{ }^{\circ} \mathrm{C}$ for short annealing times showed an average separation of the clusters with the nearest neighbors of approximately $200 \mathrm{~nm}$ to $300 \mathrm{~nm}$, which agrees well with a roughening mechanism based on a fermionic Casimir interaction rather than a Van der Waals interaction. This quantum-mechanical mechanism is expected to dominate in metals but not in insulators.

\section{REFERENCES}

${ }^{1}$ M. W. Finnis, J. Phys.: Condens. Matter 8, 5811 (1996).

${ }^{2}$ M. Baumer and H. Freund, Prog. Surf. Sci. 61, 127 (1999).

${ }^{3}$ M. Zinke-Allmang, Thin Solid Films 346, 1 (1999).

${ }^{4}$ R. Seemann, S. Herminghaus, and K. Jacobs, Phys. Rev. Lett. 86, 5534 (2001).

${ }^{5}$ R. Xie, A. Karim, J. F. Douglas, C. C. Han and R. A. Weiss, Phys. Rev. Lett. 81, 1251 (1998).

${ }^{6}$ K. Morgenstern, G. Rosenfeld and G. Comsa, Phys. Rev. Lett. 76, 2113 (1996).

${ }^{7}$ K. Morgenstern, G. Rosenfeld, G. Comsa, M. R. Sorensen, B. Hammer, E. Laegsgaard and F. Besenbacher, Phys. Rev. B. 63, 0454121 (2001).

${ }^{8}$ J. M. Wen, S. L. Chang, J. W. Burnett, J. W. Evans and P. A. Thiel, Phys. Rev. Lett. 73, 2591 (1994).

9 J. B. Hannon, C. Klunker, M. Giesen, H. Ibach, N. C. Bartelt, and J. C. Hamilton, Phys. Rev. Lett. 79, 2506 (1997).

${ }^{10}$ N. C. Bartelt, W. Theis and R. M. Tromp, Phys. Rev. B 54, 11741 (1996).

${ }^{11}$ L. Fitting, M. C. Zeman, W. C. Yang and R. J. Nemanich, J. Appl. Phys. 93, 4180 (2003).

${ }^{12}$ M. R. Krause, A. Stollenwerk, M. Licurse and V. P. LaBella, J. Vac. Sci. Technol. A 24, 1480 (2006).

${ }^{13}$ S. Kodambaka, V. Petrova, S. V. Khare, D. Gali, A. Rockett, I. Petrov and J. E. Greene, Phys. Rev. Lett. 89, 176102 (2002). 
${ }^{14}$ Woei Wu Pai, Anna K. Swan, Zhenyu Zhang and J. F. Wendelken, Phys. Rev. Lett. 79, 3210 (1997).

${ }^{15}$ W. C. Yang, M. Zeman, H. Ade and R. J. Nemanich, Phys. Rev. Lett. 90, 136102 (2003).

${ }^{16}$ I. M. Lifshitz and V. V. Slyosov, J. Phys. Chem. Solids 19, 35 (1961).

${ }^{17}$ K. Shorlin, S. Krylov and M. Zinke-Allmang, Physica A 261, 248 (1998).

18 J. A. Marqusee, J. Chem. Phys. 81, 976 (1984).

19 J. G. McLean, B. Krishnamachari, D. R. Peale, E. Chason, J. P. Sethna and B. H. Cooper, Phys. Rev. B 55, 1811 (1997).

20 J. Becker, G. Grun, R. Seemann, H. Mantz, K. Jacobs, K. R. Mecke and R. Blossey, Nature Mater. 2, 59 (2003).

${ }^{21}$ J. Espinosa, H. Shi and D. Lederman, J. Appl. Phys. 99, 023516 (2006)

${ }^{22}$ X. Yang and S. Perry, Surf. Sci. 506, L261 (2002).

${ }^{23}$ S. Herminghaus, K. Jacobs, K. Mecke, J. Bischof, A. Fery, M. Ibn-Elhaj, S. Schlagowski, Science 282, 916 (1998).

${ }^{24}$ K. Thurmer, E. D. Williams and J. E. Reutt-Robey, Phys. Rev. B. 68, 155423 (2003).

${ }^{25}$ H. Shi and D. Lederman, J. Appl. Phys. 87, 6095 (2000).

${ }^{26}$ L. Vitos, A. V. Ruban, H. L. Skriver and J. Kollar, Surf. Sci. 411, 186 (1998).

27 J. Friedel, The physics of metals (ed. J. M. Ziman), p. 494, Cambridge University Press, New York (1969).

${ }^{28} \mathrm{H}$. Luth, Solid Surfaces, Interfaces and Thin Films, $4^{\text {th }}$ edition, Springer-Verlag, Berlin (2001).

${ }^{29}$ I. M. Lifshitz, and V. V. Slyosov, J. Phys. Chem. Solids 19, 35 (1961).

30 J. A. Marqusee and J. Ross, J. Chem. Phys. 80, 536 (1984).

${ }^{31}$ K. Shorlin, S. Krylov and M. Zinke-Allmang, Physica A 261, 284 (1998).

32 B. Krishnamachari, J. McLean, B. Cooper and J. Sethna, Phys. Rev. B 54, 8899 (1996).

${ }^{33}$ J. G. McLean, B Krishnamachari, D. R. Peale, E. Chason, J. P. Sethna and B. H. Cooper, Phys. Rev. B 55, 1811 (1997). 
${ }^{34}$ M. R. Krause, A. Stollenwerk, M. Licurse and V. P. LaBella, J. Vac. Sci. Technol. A 24, 148 (2006).

${ }^{35}$ R. Limary and P. F. Green, Langmuir 15, 5617 (1999).

${ }^{36}$ H. Sadan and W. D. Kaplan, J. Mater. Sci. 41, 5099 (2006).

${ }^{37}$ A. Vrij and J. Th. G. Overbeek, J. Am. Chem. Soc. 90, 3074 (1968).

${ }^{38}$ R. Seemann, S. Herminghaus, and K. Jacobs, Phys. Rev. Lett. 86, 5534 (2001).

${ }^{39}$ W. W. Mullins, Interface Science 9, 9 (2001).

${ }^{40}$ A. Sharmar and G. Reiter, Phase Transitions 75, 377 (2002).

${ }^{41}$ H. B. G. Casimir, Proc. K. Ned. Akad. 51, 79 (1948).

${ }^{42}$ S. K. Larmoreaux, Phys. Rev. Lett. 78, 5 (1997).

${ }^{43}$ A. Lambrecht, Physics World, September, p. 3 (2002).

${ }^{44}$ E. M. Lifshitz, J. Exp. Theor. Phys. 29, 94 (1955).

45 J. N. Israelachvili, Intermolecular and Surface Forces, Second Edition, Academic Press, London, 1991.

${ }^{46}$ A. Bulgac and A. Wirzba, Phys. Rev. Lett. 87, 120404 (2001).

${ }^{47}$ A. Wirzba, A. Bulgac and P. Magierski, J. Phys. A: Math. Gen. 39, 6815 (2006).

${ }^{48}$ L. Golubovic, Personal Communication.

49 J. Becker, G. Grun, R. Seeman, H. Mantz, K. Jacobs, K. R. Mecke and R. Blossey, Nature Mater. 2, 29 (2003).

${ }^{50}$ G. Reiter, P. Auroy and L. Auvray, Macromolecules 29, 2150 (1996).

${ }^{51}$ P. Muller-Bushbaun, J. Phys.: Condens. Matter 15, 1549 (2003).

${ }^{52}$ C. Lorenz, Thesis, University of Mainz (1997).

${ }^{53}$ G. Levi and W. D. Kaplan, Acta Mater. 51, 2793 (2003).

${ }^{54}$ F. Dwikusuma, D. Saulys and T. F. Kuech, J. Electrochem. Soc. 149, G603 (2002).

${ }^{55}$ MDC Operation and Service Manual of Maxtek Film Deposition Controller MDC-360. 
56 Ch. Morawe, A. Stierle, N. Metoki, K. Bröhl and H. Zabel, J. Magn. Magn. Mater. 102, 223 (1991).

${ }^{57}$ Omicron VT SPM user's manual.

${ }^{58}$ R. Konnur, K. Kargupta and A. Sharma, Phys. Rev. Lett. 84, 931 (2000).

${ }^{59}$ R. N. Bracewell, "The Fourier Transform and its Applications", $2^{\text {nd }}$ Edition (McGraw-Hill, New York, 1986). 


\section{VITA \\ Jorge Espinosa}

\section{EDUCATION}

PhD. Degree in Physics

August 2007

West Virginia University. Morgantown, WV

Master of Science in Physics

May 2005

West Virginia University. Morgantown, WV

Bachelor of Science in Applied Physics

July 1993

Universidad de Santiago. Santiago of Chile

\section{RESEARCH EXPERIENCE}

Research Assistant:

West Virginia University, Physics Department

$2001-2006$

Research Assistant:

Pontificia Universidad Catolica de Chile, Santiago of Chile

$1997-2000$

Detector Engineer:

European Southern Observatory. Cerro La Silla, Chile

$1995-1996$

\section{TEACHING EXPERIENCE}

\section{Teaching Assistant:}

West Virginia University, Morgantown WV. USA

2000-2006

Part Time Lecturer:

Universidad Federico Santa Maria, Santiago of Chile

1999

Teaching Assistant:

Catholic University of Chile. Santiago of Chile

1998-1999 